\title{
OASIS integral-field spectroscopy of the central kpc in 11 Seyfert 2 galaxies
}

\author{
I. Stoklasová ${ }^{1,2}$, P. Ferruit ${ }^{3}$, E. Emsellem ${ }^{3}$, B. Jungwiert ${ }^{1}$, E. Pécontal ${ }^{3}$, and S. F. Sánchez ${ }^{4}$ \\ 1 Astronomical Institute of the Academy of Sciences of the Czech Republic, v.v.i., Boční II 1401, 14131 Prague, Czech Republic \\ e-mail: ivana@sirrah.troja.mff.cuni.cz \\ 2 Faculty of Mathematics and Physics, Charles University in Prague, Ke Karlovu 3, 12161 Prague, Czech Republic \\ 3 Université de Lyon, France; Université Lyon 1, 69007; CRAL, Observatoire de Lyon, 69230 Saint-Genis-Laval; CNRS, UMR 5574; \\ ENS de Lyon, France \\ 4 Centro Astronómico Hispano-Alemán, Calar Alto, CSIC-MPG, C/Jesús Durbán Remón 2-2, 04004 Almería, Spain
}

Received 24 October 2008 / Accepted 15 January 2009

\begin{abstract}
Aims. We examine the physical conditions of ionised gas in the central kiloparsec(s) of nearby Seyfert galaxies. Our aim is a detailed two-dimensional mapping of optical spectroscopic properties, searching for features in the emission structure and in gas kinematics which would be common to all the active galactic nuclei (AGN) or their class, and which would help us to understand the origin and nature of the circumnuclear gas, and its role in the AGN.

Methods. We map narrow-line regions (NLRs) of 11 nearby Seyfert 2 galaxies with the optical integral-field spectrograph OASIS mounted at CFHT. We model emission-line profiles of 5 forbidden-line doublets and 2 Balmer lines, correcting for the underlying stellar absorption by reconstructing stellar spectra with synthetic evolutionary stellar population models.

Results. For each of the 11 targets, we present 2D maps of surface brightness in the observed emission lines, diagnostic line intensity ratios, gas kinematics (mean line-of-sight velocity and velocity dispersion), electron density, and interstellar reddening, and we plot spatially resolved spectral-diagnostic diagrams. The stellar data are represented by maps of mean line-of-sight (LOS) velocities and of the relative mass fractions of the young stellar populations. The gas velocity fields in $80 \%$ of the sample exhibit twisted Sshaped isovelocity contours, which are signatures of non-circular orbits and indicate non-axisymmetric gravitational potentials, gas motions out of the galactic plane, or possible outflows and inflows. Based on the kinematic measurements, we identified a possible nuclear ring or radial gas flow in NGC 262 (Mrk 348), not reported before. Eight of the eleven observed objects exhibit strongly asymmetric or multi-component emission-line profiles, in most cases confined to an elongated region passing through the galactic centre, perpendicular to the major axis of emission.
\end{abstract}

Key words. galaxies: active - galaxies: Seyfert - methods: observational - methods: data analysis - techniques: spectroscopic galaxies: kinematics and dynamics

\section{Introduction}

In the search for the fuelling mechanism of active galactic nuclei (AGN) and its trigger, no significant differences have been found between the host galaxies of AGN and their quiescent counterparts. Among others, no excess of galactic bars has been confirmed in active galaxies (Martini et al. 2003; Laurikainen et al. 2004). On the other hand, recent studies of galactic centres have reported an excess of infrared isophotal twists (Hunt \& Malkan 2004) and of twisted isovelocity contours in gas (Dumas et al. 2007), which may imply presence of non-axisymmetric potentials (Schinnerer et al. 2000). Focus on gas kinematics in the nuclear regions thus seems to be the key to understanding the interplay between the host galaxy and the active nucleus, even though interpretation of the phenomena on very different spatial scales needs to be done with extreme care. Detailed spatially resolved spectroscopic observations of the nuclear regions in large samples of galaxies, followed by proper modelling are hence necessary.

The Narrow-Line Regions (NLR) represent low-density gas ionised by the central AGN (e.g., Morse et al. 1996; Evans et al. 1999) and extending beyond the central dusty torus. The classical NLRs lie within 10-100 pc scales and their kinematics is often perturbed by energy injection from the AGN while gas motions in the so-called extended NLRs (ENLRs), reaching to $\mathrm{kpc}$ and $10 \mathrm{kpc}$ scales, are thought to be predominantly gravitational. The ENLRs are convenient observational targets for spatially resolved imaging and spectroscopy, especially in nearby Seyfert galaxies. They play a crucial role in attempts to understand the AGN structure and evolution, and in testing of the Unified Models of AGN (Antonucci 1993; Urry \& Padovani 1995).

By completing narrow-band imaging and aperture spectroscopy observations of large samples of Seyfert galaxies, robust statistical analyses of NLR properties have been achieved (Mulchaey et al. 1996a; Ho et al. 1997; Schmitt et al. 2003a). Similar surveys with modern spatially resolved spectroscopic techniques are necessary to obtain more complex information and achieve a qualitatively new level of conclusions. As an example, we mention the controversy in optical imaging studies by Mulchaey et al. (1996a); and Schmitt et al. (2003a), who tested predictions of the Unified Model with respect to the NLR size and morphology, and obtained results that cast doubts on the simple model of ionisation of ambient gas by a cone of 
Table 1. List of observed Seyfert galaxies - basic data.

\begin{tabular}{lllllllcc}
\hline \hline Object & $\begin{array}{l}\text { Alternative } \\
\text { name }\end{array}$ & $\begin{array}{l}\text { RA } \\
{[\mathrm{h} \mathrm{m} \mathrm{s}]}\end{array}$ & $\begin{array}{l}\text { Dec } \\
{[\mathrm{d} \mathrm{m} \mathrm{s}]}\end{array}$ & $\begin{array}{l}\text { Hubble } \\
\text { type }\end{array}$ & Redshift $z$ & $\begin{array}{l}\text { Velocity } c z \\
{\left[\mathrm{~km} \mathrm{~s}^{-1}\right]}\end{array}$ & $\begin{array}{c}D_{\mathrm{L}} \\
{[\mathrm{Mpc}]}\end{array}$ & $\begin{array}{c}\text { Ang.scale } \\
{\left[\mathrm{pc} /{ }^{\prime \prime}\right]}\end{array}$ \\
\hline Mrk 34 & MCG +10-15-104 & 103408.6 & +600152 & Spiral & 0.0505 & $15140 \pm 90$ & 218 & 956 \\
Mrk 622 & UGC 04229 & 080741.0 & +390015 & S0 & 0.0232 & $6964 \pm 11$ & 99.6 & 461 \\
Mrk 1066 & UGC 02456 & 025958.6 & +364914 & (R)SB(s)0+ & 0.0120 & $3605 \pm 22$ & 47.3 & 224 \\
NGC 262 & Mrk 348 & 004847.1 & +315725 & SA(s)0/a & 0.0150 & $4507 \pm 4$ & 58.2 & 274 \\
NGC 449 & Mrk 1 & 011607.2 & +330522 & (R')S? & 0.0159 & $4780 \pm 2$ & 62.3 & 293 \\
NGC 2273 & Mrk 620 & 065008.6 & +605045 & SB(r)a: & 0.0061 & $1840 \pm 4$ & 25.8 & 124 \\
NGC 2992 & MCG -02-25-014 & 094542.0 & -141935 & Sa pec & 0.0077 & $2311 \pm 4$ & 36.6 & 175 \\
NGC 3081 & IC 2529 & 095929.5 & -224935 & (R )SAB(r)0/a & 0.0080 & $2391 \pm 3$ & 37.7 & 180 \\
NGC 4388 & UGC 07520 & 12 25 46.7 & +123944 & SA(s)b: sp & 0.0084 & $2524 \pm 1$ & $16.7^{(*)}$ & $81^{(*)}$ \\
NGC 5728 & MCG -03-37-005 & 144223.9 & -171511 & (R) SAB(r)a & 0.0094 & $2804 \pm 20$ & 41.9 & 199 \\
NGC 5929 & UGC 09851 & 152606.1 & +414014 & Sab: pec & 0.0083 & $2492 \pm 8$ & 35.7 & 170 \\
\hline
\end{tabular}

Adopted from the NASA/IPAC Extragalactic Database (NED). The cosmological scales assume $H_{0}=73 \mathrm{~km} \mathrm{~s}^{-1} \mathrm{Mpc}^{-1}, \Omega_{\mathrm{M}}=0.27, \Omega_{\Lambda}=0.73$. $D_{\mathrm{L}}$ : luminosity distance; Ang.scale: angular scale. (*) Values from Yasuda et al. (1997), with NGC 4388 considered inside the Virgo cluster.

radiation. However, both studies are affected by the limitations typical of pure imaging: these include the survey sensitivity or contamination by other emission sources, the most important being gas ionised by young, massive stars that have been found in centres of many Seyfert galaxies (e.g., Schmitt et al. 1999; Cid Fernandes et al. 2004; Boisson et al. 2004).

Spatially resolved spectroscopy overcomes many of these difficulties: ratios of forbidden and permitted emission-line intensities at different locations help us to discriminate between the ionisation sources and to establish a boundary of the AGNionised NLR, using ionisation models (see e.g., Bennert et al. 2006a, for a discussion and application). Also, spatially resolved kinematics derived from the emission lines provides an important insight into the three-dimensional structure and dynamics of the central emitting regions. The wealth of information contained in such spectroscopic data thus allows us to address a number of questions related to the NLRs, which are important for reconstructing the global picture of AGN. An efficient way of acquiring simultaneously the spatially resolved optical or infrared (IR) spectroscopic data is provided by the integral-field spectroscopy (IFS). Integral-field units (IFUs) are becoming a standard type of equipment at large telescopes, available with a wide variety of technical parameters, such as spectral range, spectral and spatial resolution, and field of view.

We present our mapping of the central $\sim$ kiloparsec(s) of 11 nearby Seyfert 2 galaxies observed with the optical integralfield spectrograph OASIS mounted at the $3.6 \mathrm{~m}$ Canada-FranceHawaii Telescope (CFHT). Now mounted at William Herschel Telescope (WHT), OASIS is an IFU well-suited to highresolution observations over a restricted field of view (FOV), for a wide range of spectral modes. Its use for detailed spectroscopic mapping of the NLRs and the central parts of the ENLRs is thus advantageous for understanding the complex spatial variations in the physical properties of the circumnuclear ionised gas, and the implications for the AGN model.

For the observed emission regions, we plot maps of derived quantities such as the surface brightness in two Balmer lines and five doublets of forbidden emission lines, the computed line spectral-diagnostic ratios, the kinematic properties, the electron densities, and the interstellar reddening. We plot spatially resolved spectral-diagnostic diagrams for each object where possible. We provide details of the individual Seyfert 2 nuclei in Sect. 4, confronting our observations with results found in the literature. The complete sample is discussed in Sect. 5, where we draw conclusions about various structural and kinematic results.

\section{Observations and data reduction}

\subsection{Observations}

The selected 11 targets (Table 1) correspond to well-known Seyfert galaxies with previously published photometric and/or spectroscopic data. Their redshifts are in the range $0.006<z<$ 0.051 , corresponding to distances of $17-220 \mathrm{Mpc}$. Observations were performed in the years 2000-2002, with the lenslet-array OASIS spectrograph constructed at the Centre de Recherche Astrophysique de Lyon (CRAL), France, mounted at CFHT (Mauna Kea) in the F/8 Cassegrain focus. Two spectral domains were explored, with either high $\left(0.27^{\prime \prime}\right)$ or low $\left(0.41^{\prime \prime}\right)$ spatial sampling, and corresponding fields of view of $10^{\prime \prime} \times$ $8^{\prime \prime}$ and $15^{\prime \prime} \times 12^{\prime \prime}$, respectively. Each of the modes provided approximately a thousand spectra per object per spectral domain. The two spectral modes covered the wavelength ranges of 4760-5558 $\AA$ with $2.15 \AA$ dispersion ("MR1" spectral mode), and 6210-7008 $\AA$ with $2.17 \AA$ dispersion ("MR2" spectral mode).

Table 2 specifies the spectral and spatial configurations used for each member of the entire observed set. An overview of all the configurations of OASIS is available at the dedicated web pages of the instrument and at the CFHT pages. Details of the instrumental concept are to be found in Bacon et al. (1995), which describes the TIGER spectrograph, a direct predecessor of OASIS.

\subsection{Data reduction}

The data were reduced with the dedicated XOasis software (Pécontal-Rousset et al. 2004a,b). The standard reduction procedure includes bias and dark subtraction (CCD), extraction of the spectra using a fitted mask model, wavelength calibration, low-frequency flat-fielding, spectro-spatial flat-fielding, cosmicray removal, homogenisation of the spectral resolution over the field, sky subtraction, and flux calibration using observations of the photometric standard stars. Multiple exposures were merged and mosaiced, truncating the wavelength domain to a common range in the different fields, and combining the spectra (both signal and noise) with optimal weights and renormalisation. The data cubes were resampled to a common spatial scale on a rectangular grid (from the original array of hexagonal lenses) with either $0.25^{\prime \prime}$ or $0.4^{\prime \prime}$ spacing depending on the adopted spatial sampling. Orientation was defined to be north up, east to the left. 
Table 2. List of observed Seyfert galaxies - observational parameters.

\begin{tabular}{llllrc}
\hline \hline Object & $\begin{array}{l}\text { Observation } \\
\text { date }\end{array}$ & $\begin{array}{l}\text { Integration time } \\
\text { in MR1 [s] }\end{array}$ & $\begin{array}{l}\text { Integration time } \\
\text { in MR2 [s] }\end{array}$ & $\begin{array}{c}\text { Spatial sampling } \\
\text { MR1 }\end{array}$ & $\begin{array}{c}\text { Spatial sampling } \\
\text { MR2 }\end{array}$ \\
\hline Mrk 34 & $14-15 / 03 / 2001$ & $1 \times 2700$ & $2 \times 2700$ & HR & LR \\
Mrk 622 & $24 / 11 / 2000$ & $2 \times 1800$ & $2 \times 1800$ & HR & HR \\
Mrk 1066 & $22 / 11 / 2000$ & $3 \times 1800+1 \times 1200$ & $2 \times 1800$ & HR & HR \\
NGC 262 & $25 / 11 / 2000$ & $2 \times 1800$ & $2 \times 1800$ & HR & HR \\
NGC 449 & $24 / 11 / 2000$ & 3 fields $\times 2 \times 1800$ & 3 fields $\times 2 \times 1800$ & HR & HR \\
NGC 2273 & $24 / 11 / 2000$ & $2 \times 1800$ & - & LR & HR \\
NGC 2992 & $16-17 / 03 / 2001$ & $1 \times 1800+1 \times 2700$ & $2 \times 1800$ & LR & - \\
NGC 3081 & $17 / 01 / 2002$ & $2 \times 3600$ & - & LR & LR \\
NGC 4388 & $17 / 01 / 2002$ & $3 \times 3300$ & - & LR & LR \\
NGC 5728 & $15 / 03 / 2001$ & $2 \times 1800$ & $1 \times 1800+1 \times 2700$ & LR & - \\
NGC 5929 & $16 / 03 / 2001$ & $2 \times 2700$ & - & & \\
\hline
\end{tabular}

The abbreviations used: MR1: spectral mode of wavelength range $(4760,5558) \AA$, dispersion $2.15 \AA$ /pixel, resolving power 1210; MR2: spectral mode of wavelength range $(6210,7008) \AA$, dispersion $2.17 \AA$ /pixel, resolving power 1525 ; HR: high spatial resolution mode with sky sampling $0.27^{\prime \prime}$, field of view $10.4^{\prime \prime} \times 8.3^{\prime \prime}$; LR: low spatial resolution mode, sky sampling $0.41^{\prime \prime}$, field of view $15^{\prime \prime} \times 12^{\prime \prime}$.

\section{Data analysis}

The results presented in this paper were obtained by a two-stage analysis procedure: stellar-population modelling and emissionline modelling. The OASIS survey that we present was designed preferentially for the study of the gas dynamics, and the signalto-noise ratio $(S / N)$ of the acquired data was therefore calculated for optimal results in the gas emission lines. A simplified stellarpopulation modelling, with the primary goal of correcting the Balmer lines of hydrogen for underlying stellar absorption, permitted us to investigate both the stellar kinematics and the age of stellar populations.

Methods for the simultaneous fitting of stellar and emission gas spectra were recently developed by the SAURON consortium (see e.g., Sarzi et al. 2006; McDermid et al. 2006) and others (e.g., Sánchez et al. 2007). We briefly tested this software on our data and found that the low $S / N$ in our stellar lines prevented us from judging reliably which method was more suitable for our data. In addition, the two-stage spectral analysis, with the independent treatment of the stellar and gaseous components, is advantageous for detailed studies of complex emission-line profiles: the stellar component removed, different emission-line models with an increasing degree of precision can gradually be applied, as needed.

For each of the data-analysis steps, the large volumes of data require to a large extent automatic treatment, with a subsequent check of the results, and detailed modelling applied only to selected spectra. The current paper presents our first-order results, while detailed analysis and interpretation of selected objects is postponed to future papers dedicated to individual objects in the sample.

\subsection{Stellar populations}

We modelled the stellar component of spectra using synthetic evolutionary models from the GALAXEV library of Bruzual \& Charlot (2003). Understanding the stellar populations in Seyfert galaxies has always been a challenging problem (see e.g., Cid Fernandes et al. 2005; Raimann et al. 2003), due to the presence of strong gas emission lines, which contaminate the stellar spectra. A fairly standard approach has been to correct emission lines for stellar absorption by assuming that the nuclear stellar populations are identical to those in the outer parts of the host galaxy (e.g., Ho et al. 1997; Bennert et al. 2006a). However, evidence of young stars in the centres of Seyferts has been presented by e.g., Heckman et al. (1997), González Delgado et al. (1998), Joguet et al. (2001), Raimann et al. (2003), and Cid Fernandes et al. (2004), and therefore the use of purely old stellar populations has been shown to be insufficient even for detailed gas-emission studies, because of the need to correct reliably for the effects of stellar absorption.

Stellar-population modelling searches for a combination of template spectra from a theoretical or experimental library which reproduces best the observed galactic spectrum. As a typical inverse problem, the stellar spectrum reconstruction has a number of free parameters (such as the ages and metallicities of the individual populations, and their mass fractions), which may not be possible to constrain to form a unique solution. Intrinsic degeneracies exist between age and metallicity, and they are further enhanced by measurement uncertainties and noise, which wash away the differences between spectrally similar components, as demonstrated by Cid Fernandes et al. (2004). These authors showed for artificial data that even an accurately fitting template does not ensure a correct physical interpretation. Nevertheless, a most robust determination of population fractions is possible if larger stellar-age bins are used (defined coarsely as young $<100 \mathrm{Myr}$, intermediate $100 \mathrm{Myr}-1.4 \mathrm{Gyr}$, and old >1.4 Gyr in their study).

We adapted the stellar population study to our data quality. We were limited by the $S / N$ in stellar absorption lines, the relatively short wavelength ranges with only a few prominent absorption lines, and contamination by gas emission lines. The fitting of the stellar populations was only possible in one of the two observed spectral regions, i.e., the MR1 domain (see Sect. 2.1), which contains the $\mathrm{Mg} \mathrm{I} b$ complex at about $5170 \AA$, and iron lines Fe $5015 \AA$, Fe $5270 \AA$. Other constraints to the models were provided by the absorption-line wings of $\mathrm{H} \beta$. No such features were detected in the MR2 domain, and therefore the stellar component of spectra in this domain was inferred by extrapolating the models fitted to MR1.

From the GALAXEV models, we selected those that represent evolution of Simple Stellar Populations (SSPs) with Salpeter initial mass function (IMF), by applying Padova evolutionary tracks (e.g., Girardi et al. 2000). We based our work on the findings of Tremonti et al. (2004), and Cid Fernandes et al. (2004), who tested different sets of SSP models for reconstructing spectra of active and non-active galaxies. They defined a spectral base of 30 SSPs covering ten ages and three metallicities, which played a role of independent functions, and any 
other SSP was found to be their linear combination. The tests that we performed with this "Tremonti set" on our data revealed that the number of free parameters introduced by the theoretical combinations within the 30 SSPs was too large for our data quality, and could not be sufficiently constrained to produce a unique model, resulting in significant degeneracy with a difficult interpretation. We therefore searched for a simpler representation, and used models combining two SSPs of markedly different ages, as applied before e.g., by Canalizo et al. (2007). The even more basic option of single-population models proved to be inadequate - the models failed to reproduce the observed spectral features correctly. The two SSP models were selected to be of solar metallicity, and of ages of $11 \mathrm{Gyr}$ and $100 \mathrm{Myr}$, which were found to contribute the most even in the models to our data produced by the entire Tremonti set, and were able to describe many different types of spectra across the whole sample. The final fits differ from those obtained with the Tremonti set by up to $\sim 20 \%$ in the equivalent width of the $\mathrm{H} \beta$ absorption line and by $\leq 20 \mathrm{~km} \mathrm{~s}^{-1}$ in the mean LOS stellar velocity (Fig. 1). We also studied other possible pairs of SSP models, but they were less successful in reconstructing the $\mathrm{H} \beta$ absorption wings, the metallic lines, or predicting the $\mathrm{H} \beta$ absorption equivalent widths that differed significantly from the Tremonti set. Some SSP model combinations (such as those of ages $11 \mathrm{Gyr}$ and $600 \mathrm{Myr}$ ) proved useful for certain objects, but the best-fit solution did not differ significantly from the final pair of SSPs (11 Gyr and $100 \mathrm{Myr}$ ), and did not provide optimal solutions for the remainder of the sample.

The best-fit model stellar template for each observed spectrum produced from a linear combination of selected SSPs was determined by using the penalised pixel-fitting code of Cappellari \& Emsellem (2004), and a careful masking of emission lines. For the purposes of inferring both stellar kinematics and stellar population ages reliably, we increased the signal-tonoise ratio by rebinning the FOV with equi-mass Voronoi tessellation (Cappellari \& Copin 2003) into irregular compact cells of uniform $S / N=50$ across the FOV. For other applications employing the stellar model to correct the gas emission, we modelled all the individual observed spectra, without losing spatial resolution.

\subsection{Emission lines}

The two observed spectral domains MR1 and MR2 contain two Balmer lines $(\mathrm{H} \alpha$ and $\mathrm{H} \beta)$ and five forbidden-line doublets of metal ions that represent some of the most fundamental spectral diagnostics for deriving physical parameters of the ionised gas: [O III] $\lambda \lambda 4959,5007,[\mathrm{~N}$ I] $\lambda \lambda 5198,5299, \quad[\mathrm{O} \mathrm{I}] \lambda \lambda 6300,6364$, [N II] $\lambda \lambda 6548,6583$, and [S II] $\lambda \lambda 6717,6731$. In addition to the intensity in individual lines, we derive their mean line-of-sight velocities and line-of-sight velocity dispersions and plot their two-dimensional distributions. Not all of the targets were observed in both spectral domains (see Table 2). Where the data are available, we computed the electron density from the [S II] doublet and estimated the interstellar reddening from the ratio of Balmer lines (Osterbrock 1989).

We modelled all narrow emission lines using single Gaussian profiles, even though asymmetric lines were expected at many locations. Cases for which we obtained large $F W H M$ values of Gaussian models led us to identify spatial regions where multicomponent fitting might be necessary. We used the fit/spec line-fitting software (Rousset 1992), which has a core in C and interface in ESO MIDAS, and permits automated fitting over entire datacubes, with constraints applied to the line intensities,
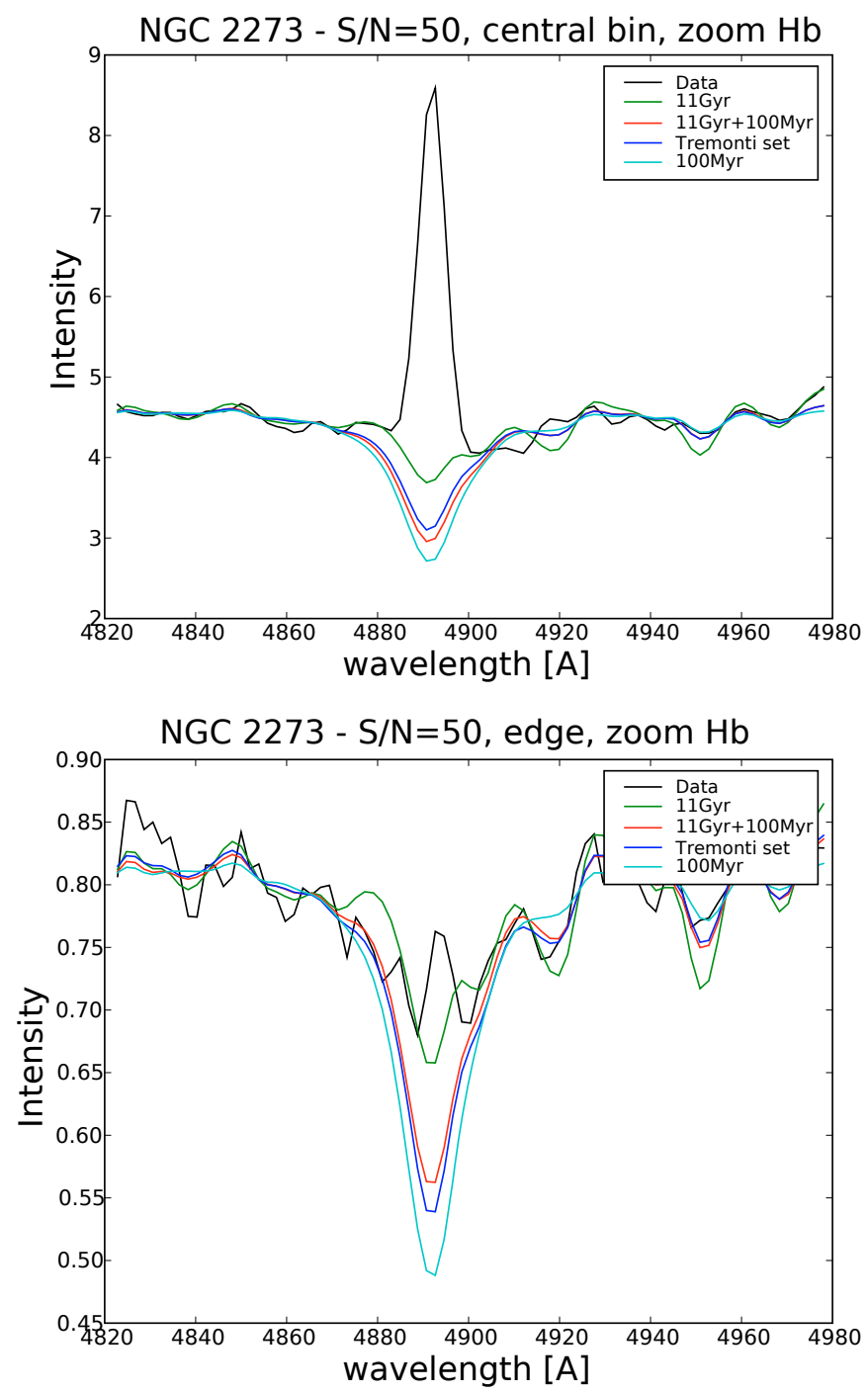

Fig. 1. NGC 2273, comparison of SSP fits in the central bin and a bin close to the FOV edge - a young stellar population (100 Myr, cyan), an old SSP (11 Gyr, green), an optimal fit resulting from the combination of two SSPs: 11 Gyr and $100 \mathrm{Myr}$ (red), and an optimal template based on the Tremonti set of SSPs (blue). Spectra binned by Voronoi tessellation at $S / N=50$.

intensity ratios, velocities, and line widths. We restricted the number of free parameters by fixing the ratios of the line intensities within the doublets to comply with the rules of atomic physics:

$0.5<[\mathrm{N} \mathrm{I}] \lambda 5198 /[\mathrm{N}$ I $] \lambda 5200<1.5$

[O III $] \lambda 5007 /[\mathrm{O}$ III] $\lambda 4959=2.88$;

[O I] $\lambda 6300 /$ [O I] $\lambda 6364=3.0$;

$[\mathrm{N}$ II $] \lambda 6583 /[\mathrm{N}$ II] $\lambda 6548=2.96$;

$0.35<[$ S II $] \lambda 6717 /[$ S II $] \lambda 6731<1.5$.

To constrain line parameters more effectively in the automated fitting process, especially for the weaker or blended lines, the emission lines were associated to form systems of assumed common kinematics. The mean LOS velocity and LOS velocity dispersion were thus determined for each of the systems, rather than the individual lines. The definition of our kinematic systems was physically and experimentally motivated. The kinematical properties of $\mathrm{O}^{++}$ions are known to differ from those of the 
low-ionisation species, due to the possibly different processes responsible for the ion formation. The first ionisation potential of elements such as oxygen, nitrogen, and sulphur is almost identical to that of hydrogen, and the ions can therefore be formed in the same conditions. And indeed, the observations show that kinematic properties of the neutral and low-ionisation species are usually similar to those of hydrogen. With this justification, we associated the weak and blended $[\mathrm{NI}]$ doublet with $\mathrm{H} \beta$ in the same system, while [O III] was treated independently. All of the MR2 lines ( $\mathrm{H} \alpha,[\mathrm{O} \mathrm{I}],[\mathrm{N}$ II], and [S II]) were considered in a common system (where possible) to assure a good quality fit to lines that are rather weak (mainly $[\mathrm{N} \mathrm{I}]$, [O I $]$, and $[\mathrm{S} \mathrm{II}]$ ) or blended ([S II] doublet lines, [N II] with $\mathrm{H} \alpha$ ).

We corrected the measured FWHM for the instrumental dispersion, which was estimated by fitting telluric lines to be (7 \pm 0.5$) \AA$. A Gaussian approximation to the line profiles was used, hence with a quadratic subtraction of the measured total and instrumental dispersions. The resulting dispersion contains an error of $0.6 \AA$ due to wavelength calibration. The differences encountered between the resultant velocity dispersion measured in $\mathrm{H} \alpha$ and in $\mathrm{H} \beta$ can be almost entirely attributed to their different data quality, far higher for $\mathrm{H} \alpha$. Where available, $\mathrm{H} \alpha$ results should therefore be considered more reliable.

\subsection{Error computation}

Uncertainties in the measurements of the mean LOS velocity, which are a function of the signal-to-noise ratio, were studied for OASIS data in detail by Ferruit et al. (2004), who fitted repeatedly artificial emission-line spectra with added noise in 500 Monte Carlo realisations for each $S / N$ value (see also McDermid et al. 2006). Their results were applied in the way recommended by the authors in the present paper. We show the corresponding error bars in one-dimensional plots of mean LOS velocities in Figs. 3-25. The effects of fitting single Gaussian functions, and of observed line-profile asymmetries were not included in this treatment. Equally, errors introduced by the subtraction of the stellar component, especially in the hydrogen lines, were not included in the error bars. An error of $16 \mathrm{~km} \mathrm{~s}^{-1}$ due to calibration was added to the computed error in the mean LOS velocity.

The random errors in line flux determination were estimated with the assumption of Gaussian line profiles, and were computed as the product of noise in the vicinity of the lines, and the line FWHM. This procedure does not include possible systematic errors due to the stellar template determination, which are difficult to quantify, as discussed in Appendix B3 of Emsellem et al. (2004). We plot error bars in the scatter plots of the derived quantities such as the line ratios and the one-dimensional cuts of electron densities, for all of which Gaussian error propagation was applied. For electron densities, the errors were computed assuming further that the [S II] line shifts and FWHMs corresponded to those of $\mathrm{H} \alpha$, i.e., we did not consider the effects of line blending.

\section{Results for individual objects}

\subsection{General comments}

We present colour-coded maps of the line emission intensity, the mean line-of-sight velocity, and the LOS velocity dispersion in the detected emission lines for each of the Seyfert 2 galaxies in our sample. Thanks to our stellar-population modelling, the gas velocity maps can be compared with those of stars in the objects where the stellar data quality was sufficient for reliable modelling of the absorption-line profiles. The stellar modelling also yielded maps of the mass fractions of the young and old stellar populations.

Further information about the objects can be inferred from the ratios of the emission-line intensities. We derive electron density from the [S II] line ratio, as described in Osterbrock (1989), by assuming a temperature of $10000 \mathrm{~K}$ (our data do not contain a direct temperature diagnostic). We estimate the dust distribution from the Balmer line ratio. The $\mathrm{H} \alpha / \mathrm{H} \beta$ should have a fixed intrinsic value depending on the physical conditions in the environment where the recombination lines are formed. We assumed the intrinsic value of 3.1, which is typical of NLRs (Osterbrock 1989). By assuming any excess of the measured ratios to be due to dust along the line of sight, we computed the interstellar reddening $E(B-V)$.

The ratios of forbidden-line to recombination-line intensities are a measure of the ionisation and excitation state of the gas, of its density, temperature and metallicity, as well as the nature of the ionising source. We plot maps for each of the standard diagnostic ratios $[\mathrm{O} \mathrm{III}] \lambda 5007 / \mathrm{H} \beta$ (excitation map), [O I] $\lambda 6300 / \mathrm{H} \alpha$, $[\mathrm{N}$ II $] \lambda 6583 / \mathrm{H} \alpha$, and ([S II $] \lambda 6717+[\mathrm{S} \mathrm{II}] \lambda 6731) / \mathrm{H} \alpha$. More information is conveyed by the spectral-diagnostic diagrams defined by Baldwin et al. (1981); and Veilleux \& Osterbrock (1987), but in our case, these diagrams are plotted for spatially resolved data for each observed galaxy. The diagrams provide further possibilities for future detailed modelling of the ionisation structure, similar to that performed e.g., by Bennert et al. (2006b), or Allen et al. (1999) for long-slit spectroscopic data.

In our diagnostic diagrams, we compare the different existing classification schemes for emission objects commonly used in the literature, including the recent development (Kewley et al. 2001; Kauffmann et al. 2003; Stasińska et al. 2006; Kewley et al. 2006), as well as the traditional schemes, some of which were based on a single pair of lines and were reviewed by Ho et al. (1997). However, we note that the models correspond to global spectra, and one must be careful when interpreting the spatially resolved diagrams (see Stasińska 2009), which require detailed ionisation modelling for a proper understanding. Therefore, the plotted curves that correspond to the afore mentioned models play an informative role only.

All the plots presented in Figs. 2-25 show the results of single-Gaussian modelling of the emission lines. This simplification is clearly insufficient for a precise reconstruction of many spectra, where two or more components are present in each emission line, as specified below for the individual objects. The single-component fitting obviously limits the interpretation of the presented data, although the results provide a guide to the location of important structural and kinematic features in the objects. The applied modelling approach simulates the effects of lower resolution spectral data with unresolved multiple components. The resulting intensity in the lines corresponds to the integrated intensity of all components or to the intensity in the strongest component, depending on the line shape and the fit. Similarly, the fitted line-of-sight velocities trace either the motion of the strongest component at a given location or a "weighted average" of the components. The velocitydispersion maps serve as efficient indicators of locations of the multi-component spectra. While it would be impossible to inspect all spectra individually due to the enormous amounts of data, the large-dispersion areas of the FWHM maps trace the regions of complex emission-line profiles, often in unexpected locations, not necessarily at the galactic centres. 
We display profiles of the mean LOS velocity and electron density along the major axes of emission, and scatter plots of radial variations in the diagnostic line ratios as complementary information. Most of the data presented were clipped at $S / N=5$, with the exception of the weak lines [N I] and [OI], for which only at a few spatial pixels in the centre the signal-to-noise ratio exceeded the value of two, and therefore we applied clipping at $S / N=1$ to plot the two-dimensional maps. The surface brightness is in the units of $10^{-19} \mathrm{~J} \mathrm{~s}^{-1} \operatorname{arcsec}^{-2} \mathrm{~m}^{-2}$, the mean LOS velocities, and LOS velocity dispersions in $\mathrm{km} \mathrm{s}^{-1}$. The velocities were corrected for heliocentric motion using the rvcor routine of IRAF, and plotted relative to the systemic velocity of each galaxy, which was taken from NED (see Table 1).

We compared the measured fluxes of emission lines with those determined by Falcke et al. (1998). The authors provide the fluxes in [O III] and $\mathrm{H} \alpha+[\mathrm{N} \mathrm{II}]$ obtained from HST observations of Mrk 34, Mrk 348, and NGC 4388, using circular apertures of two different sizes for each object. We simulated the apertures by summing the spectra over circular regions centred on $\left[0^{\prime \prime}, 0^{\prime \prime}\right]$ and of the same radii as in the cited paper. The emission-line fluxes were measured for spectra that had a stellar component modelled by a combination of two SSPs (11 Gyr and $100 \mathrm{Myr}$ ) from Bruzual \& Charlot (2003), as described in Sect. 3.1, and with no emission-line model applied (to avoid introducing further sources of error). Results of the comparison are summarised in Table 3.

Agreement between the two data sets is closer for larger apertures, as expected. The flux that we measure in regions of radii smaller than $1^{\prime \prime}$ is affected too much by the spatial PSF (dominated by $\sim 1^{\prime \prime}$ seeing), which is an order of magnitude higher than that in the HST observations and redistributes much of the observed flux outside the selected area. Most of the fluxes that we obtained for apertures of sizes $\geq 1^{\prime \prime}$ are $10-25 \%$ lower than previously published values. Possible reasons for the discrepancy include flux-calibration errors, errors acquired during data reduction or due to an imprecise determination of the stellar-continuum level. However, it is impossible to draw conclusions about systematic differences based on only three cases.

The following subsections are devoted to the description of the individual objects, the observed features, set into the larger multi-wavelength context found in the literature. We note that we will not be restating the wavelengths of the emission lines that were listed in Sect. 3.2, except the cases where confusion would be possible.

\subsection{Individual objects}

\section{Mrk 34}

Mrk 34 is known to be a luminous FIR source with a powerful water maser (Henkel et al. 2005). Optical line emission was found to be spatially correlated with radio continuum emission, $\mathrm{PA} \sim 150^{\circ}-165^{\circ}$ (Nagar \& Wilson 1999; Falcke et al. 1998; Ulvestad \& Wilson 1984a).

Mrk 34 is the most distant object in our OASIS sample and in this case our FOV corresponds to the central $\sim 5 \mathrm{kpc}$. Because of the high redshift of the galaxy, the [S II] doublet is located outside the observed spectral range, and we are therefore unable to infer all of the spectral-diagnostic ratios, and the electron density. The observed emission in all of the observed lines has a similar morphology, extended along the direction of $\mathrm{PA} \sim 140^{\circ}-150^{\circ}$ (Fig. 2). The excitation map $[\mathrm{O} \mathrm{III}] / \mathrm{H} \beta$ has the same major axis as the [O III] emission, and more of a cone-like shape. The other diagnostic line ratios, however, have low values
Table 3. Comparison of our emission fluxes with those of Falcke et al. (1998). OASIS values correspond to fluxes corrected for absorption using a combination of SSPs as described in Sect. 3.1. Units are $10^{-19} \mathrm{~W} \mathrm{~m}^{-2}$.

\begin{tabular}{ll|ll|ll}
\hline \hline Galaxy & Aperture & \multicolumn{2}{|c|}{ OASIS data } & \multicolumn{2}{c}{ Falcke et al. (1998) } \\
& radius & {$[\mathrm{O} \mathrm{III}]$} & $\mathrm{H} \alpha+[\mathrm{N} \mathrm{II}]$ & {$[\mathrm{O} \mathrm{III}]$} & $\mathrm{H} \alpha+[\mathrm{N} \mathrm{II}]$ \\
\hline Mrk 34 & $2^{\prime \prime}$ & 4800 & 3400 & 6100 & 3600 \\
& $0.5^{\prime \prime}$ & 500 & 300 & 1300 & 700 \\
\hline Mrk 348 & $1.5^{\prime \prime}$ & 3000 & 2500 & 4100 & 2000 \\
& $0.4^{\prime \prime}$ & 1500 & 1000 & 3200 & 1500 \\
\hline NGC 4388 & $6^{\prime \prime}$ & 9800 & - & 11700 & - \\
& $1^{\prime \prime}$ & 3200 & - & 3900 & - \\
\hline
\end{tabular}

in the direction of the cones, while the maximum values are distributed along the minor axis (Fig. 2). Reddening computed from the $\mathrm{H} \alpha / \mathrm{H} \beta$ ratio possesses a maximum in the southern part of the FOV, $\sim 2 \mathrm{kpc}$ from the central maximum. The spatially resolved diagnostic diagrams indicate that the emission corresponds to a strong Seyfert regime.

The map of the mean LOS velocities in $\mathrm{H} \alpha$ (Fig. 3) provides an example of twisted S-shape isocontours. They are not evident in $[\mathrm{O} \mathrm{III}]$ and $\mathrm{H} \beta$, which may partially be caused by the smaller FOV in MR1. No galactic bar or other signature of nonaxisymmetric potential has been reported so far, and the S-shape might be the first piece of kinematical evidence of departures from axial symmetry. The low quality of the stellar data did not allow comparison of the stellar and gas velocities. The object is characterised by multi-component emission-line profiles in a region of elongated shape perpendicular to the emission major axis, as visible in the velocity dispersion map.

\section{Mrk 622}

The radio emission in this galaxy at the $20 \mathrm{~cm}$ wavelength is extended along PA $=0^{\circ}$ (Nagar et al. 1999). No prominent axis in either the optical emission or the excitation map was detected on the scale of $5^{\prime \prime}$ by Mulchaey et al. (1996a), while Schmitt et al. (2003a) reported elongation in [O III] emission at $\mathrm{PA}=55^{\circ}$ in the inner $1^{\prime \prime}$, perpendicular to the host-galaxy major axis. Shuder \& Osterbrock (1981) discovered multi-component [O III] profiles of total $F W H M=(1050 \pm 50) \mathrm{km} \mathrm{s}^{-1}$, in contrast to the nearly-Gaussian shapes of the $\mathrm{H} \alpha$, [N II], and [O II] lines of $F W H M=350 \pm 75 \mathrm{~km} \mathrm{~s}^{-1}$. They concluded that two emissionline regions of different ionisation are present, with estimated temperatures of $\sim 11000 \mathrm{~K}$ in [O III] and $\sim 6000 \mathrm{~K}$ in $\mathrm{H} \alpha$, [N II], and [O II].

The galaxy is among the most distant in our sample, which has an effect on the spatial resolution that we attain. The maps of line emission intensities do not show any particular directionality, except for the $\mathrm{H} \alpha$ and [N II] emission, which has hints of extending both to the east and the south (Fig. 4), in agreement with the optical and radio observations of Schmitt et al. (2003a); and Nagar et al. (1999). The [O III] lines are highly asymmetric and their multiple components are detected throughout the field of view (Fig. 6), with a separation between the components as large as $600 \mathrm{~km} \mathrm{~s}^{-1}$, not present in the other lines, confirming the results of Shuder \& Osterbrock (1981). Single-Gaussian modelling of [O III] thus results in large-FWHM fits, as seen in Fig. 5.

The one-component fits have a strong impact on the interpretation of the velocity field of this object. The relative intensity of the two line components in [O III] varies across the FOV, with the blue component becoming stronger in the east and the red component in the west. The fitted one-component Gaussian model 

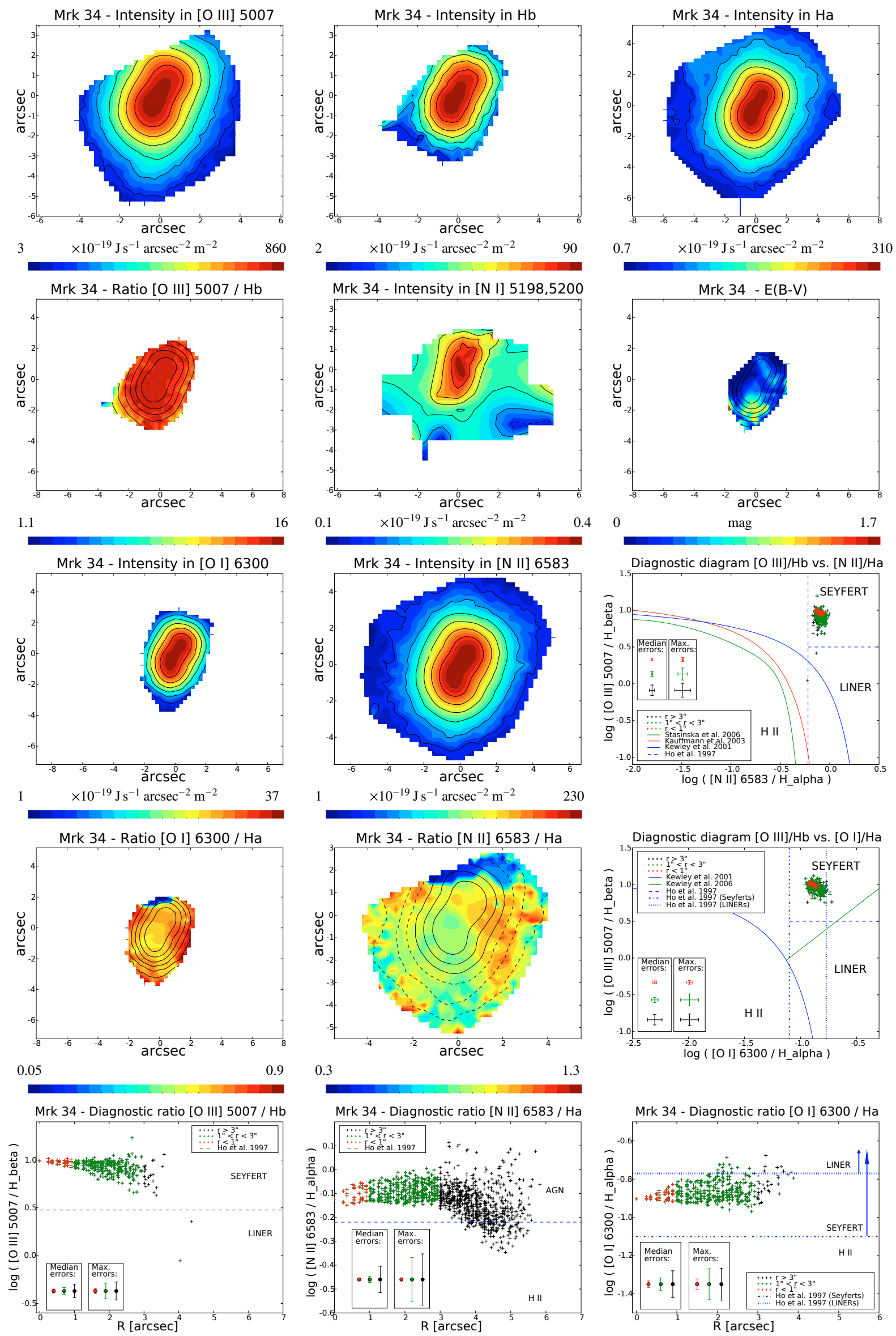

Fig. 2. Mrk 34. See detailed caption before this graphic section. Surface brightness is in $10^{-19} \mathrm{~J} \mathrm{~s}^{-1} \operatorname{arcsec}^{-2} \mathrm{~m}^{-2}$, extinction in mag in all figures. 


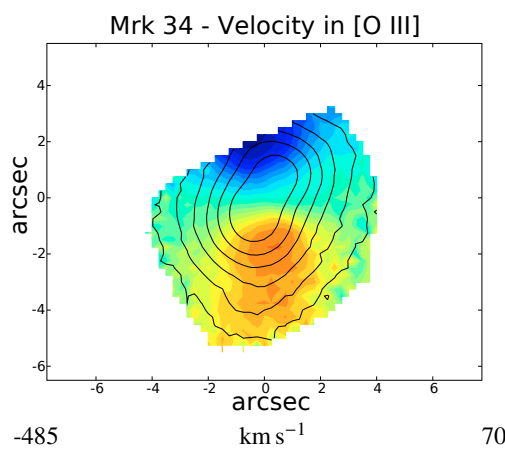

Mrk 34 - FWHM of [O III]

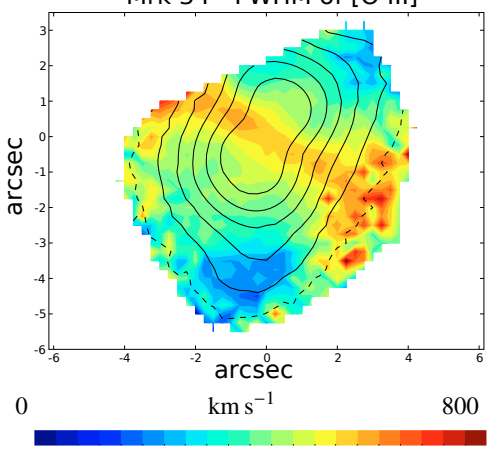

Mrk 34 Velocity $\mathrm{PA}=0.0$
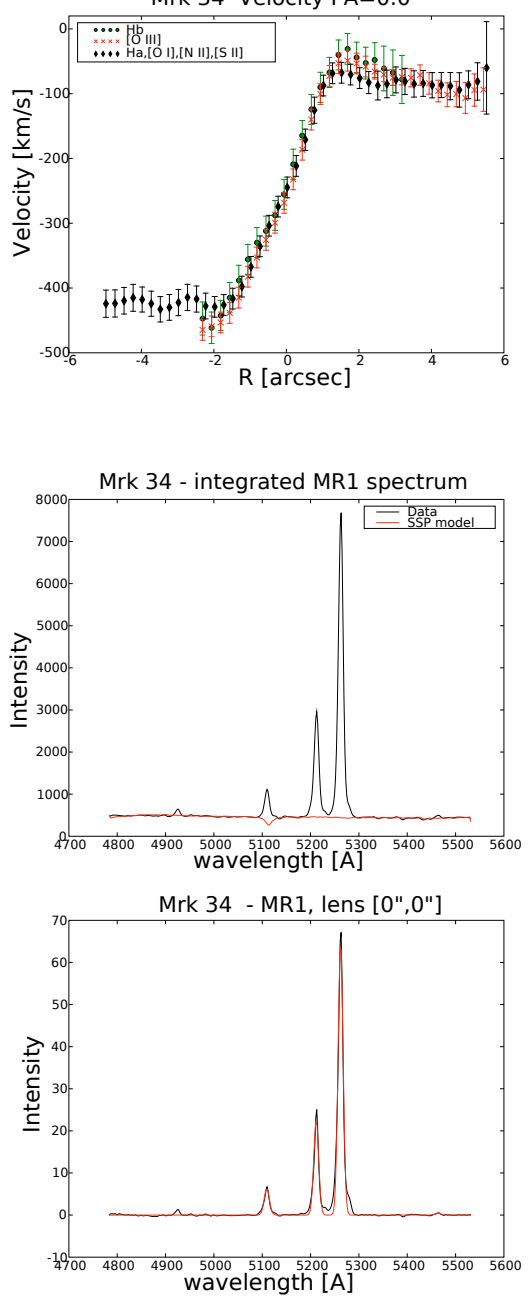
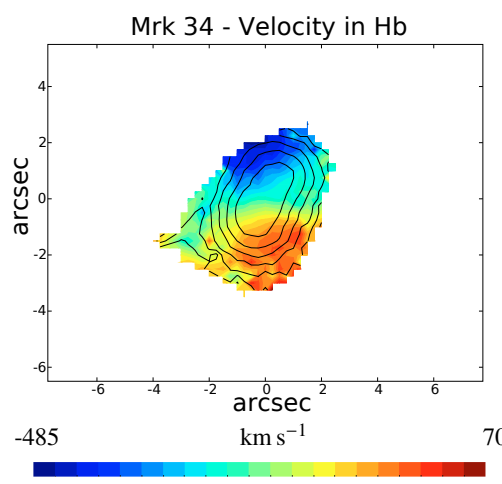

Mrk 34 - FWHM of $\mathrm{Hb}$

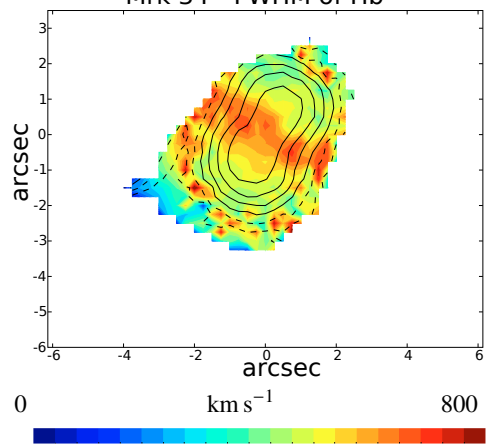

SSP mass Young/Total, $\mathrm{S} / \mathrm{N}=50$

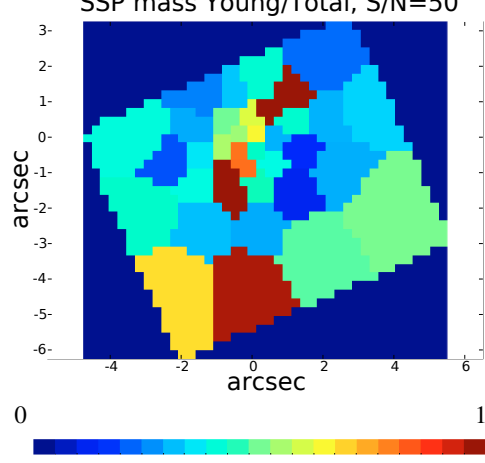

Mrk 34 - integrated MR1 spectrum

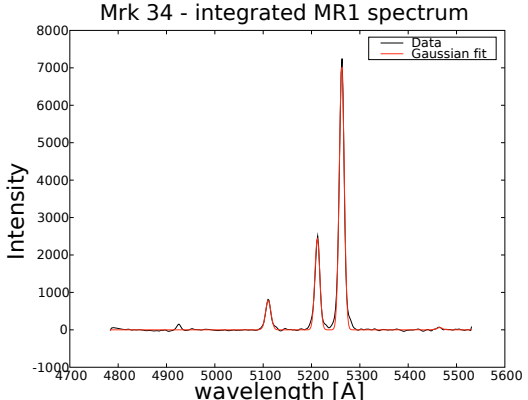

Mrk 34 - MR2, lens [0",0"]

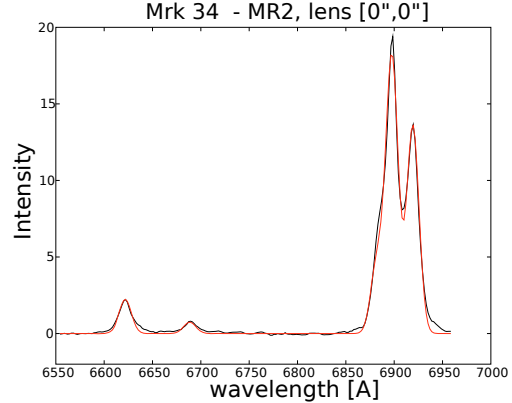

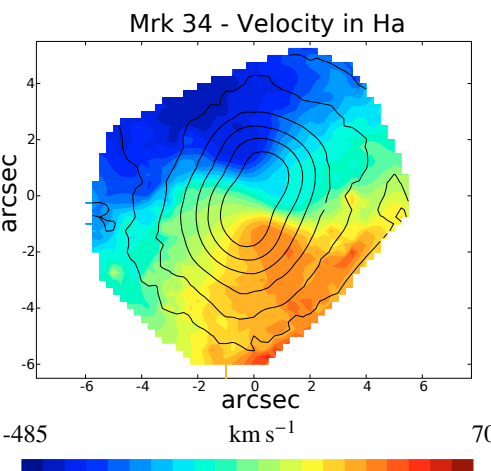

Mrk 34 - FWHM of $\mathrm{Ha}$

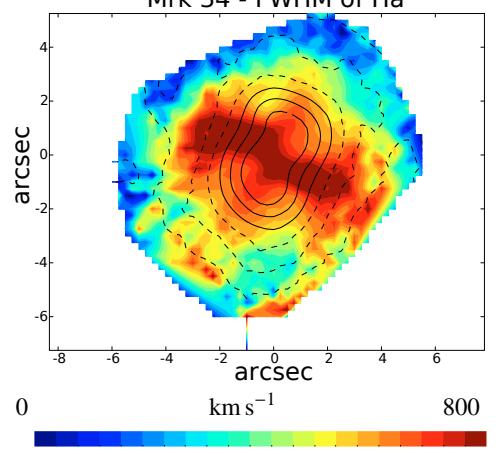

Stellar velocities $\mathrm{S} / \mathrm{N}=50$

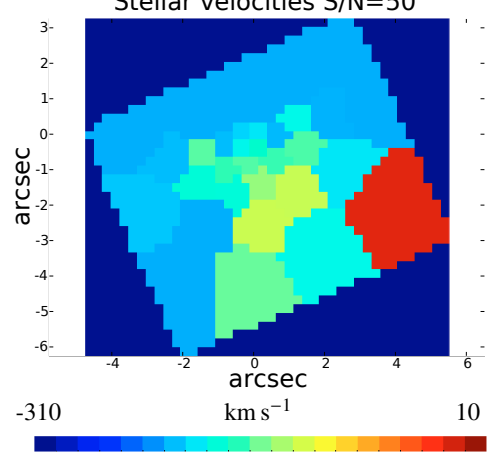

Mrk 34 - integrated MR2 spectrum
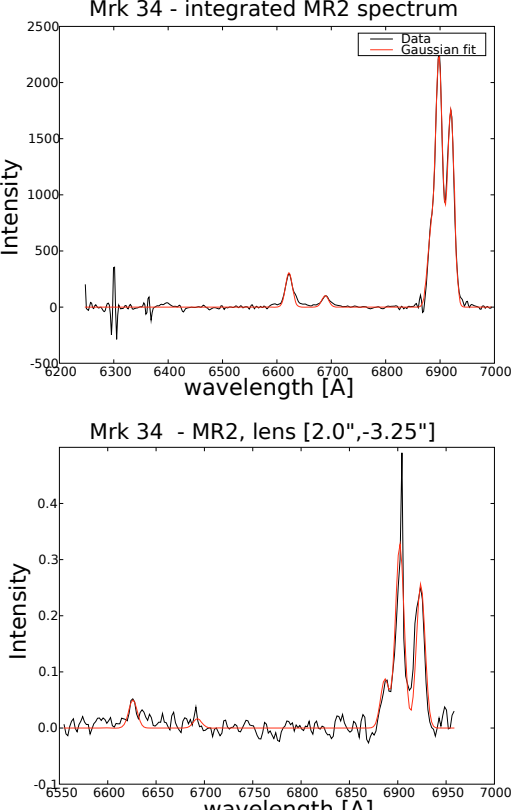

Fig. 3. Mrk 34. Mean LOS velocities and $F W H M$ are in units of $\mathrm{km} \mathrm{s}^{-1}$ in all subsequent figures. 


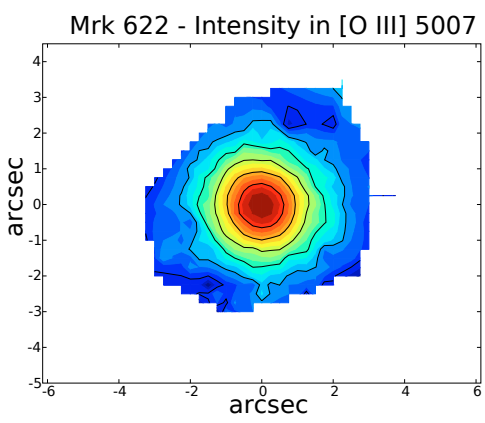

1.2

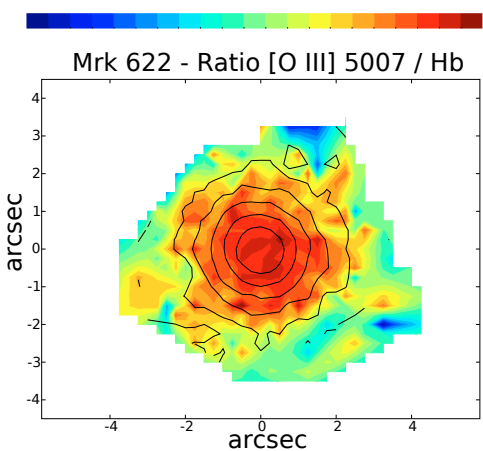

0.7
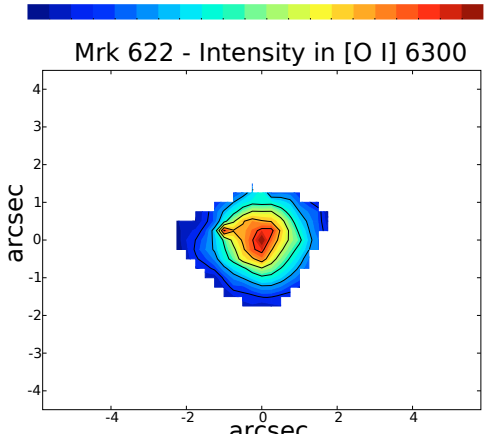

1.9

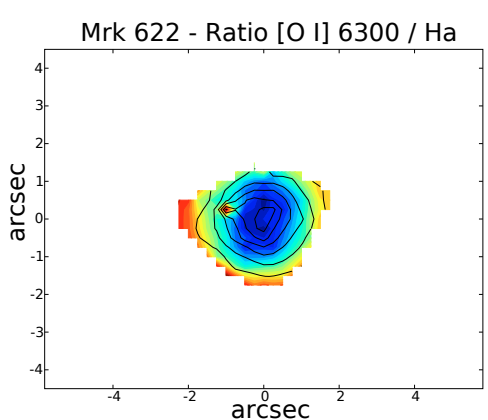

0.06

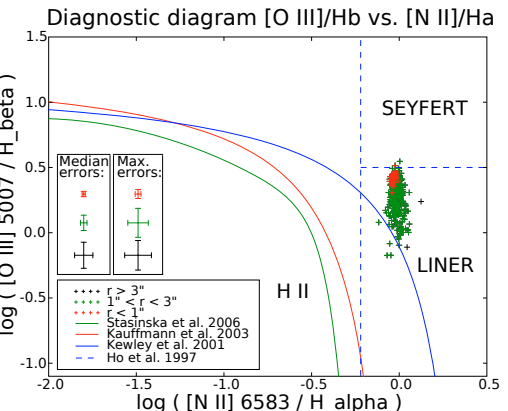

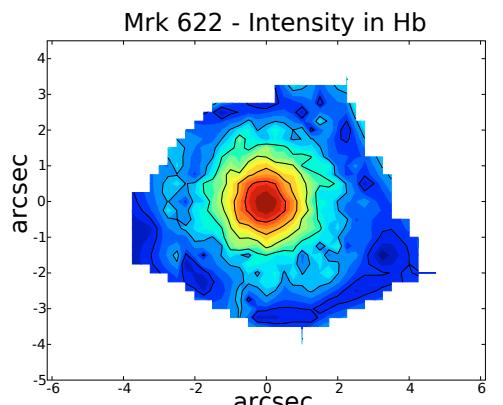

1.3

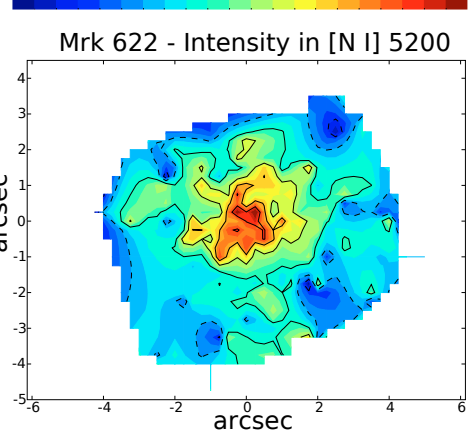

0.03

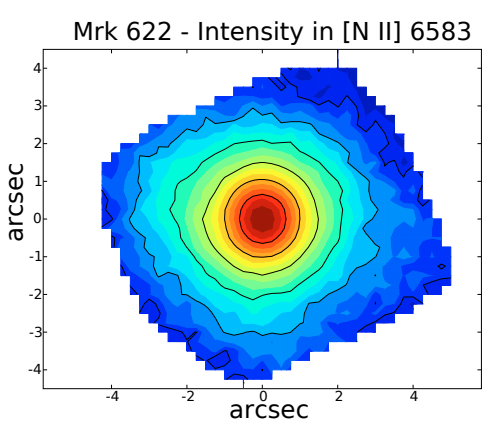

1.1

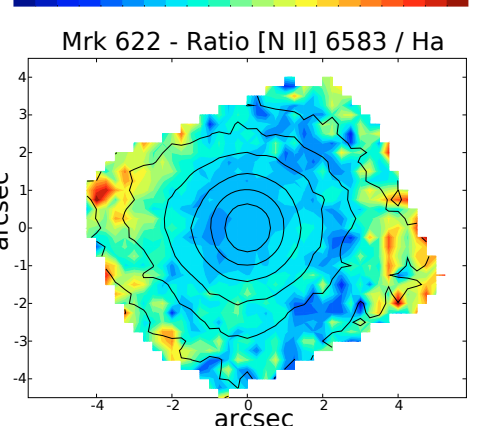

0.7

Diagnostic diagram [O III]/Hb vs. [S II]/Ha

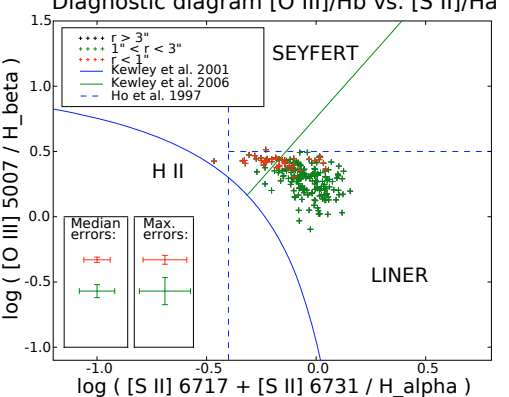

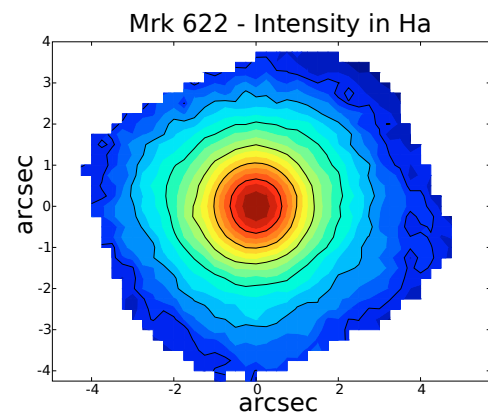

1.2

280

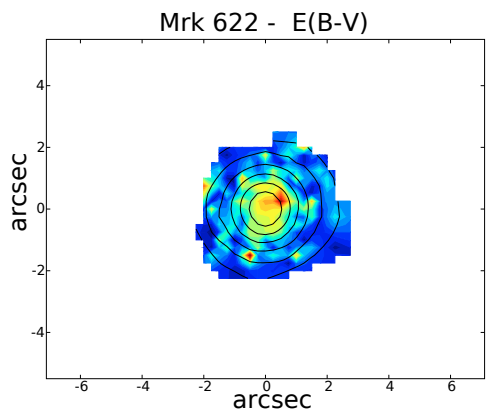

0

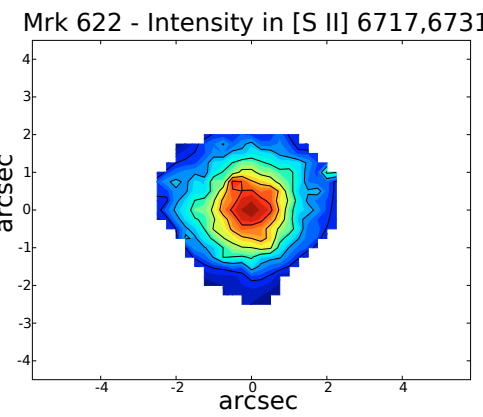

3.5

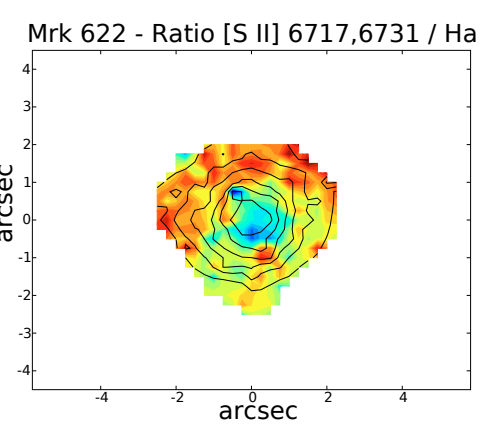

0.3

1.5

Diagnostic diagram [O III]/Hb vs. [O I]/Ha

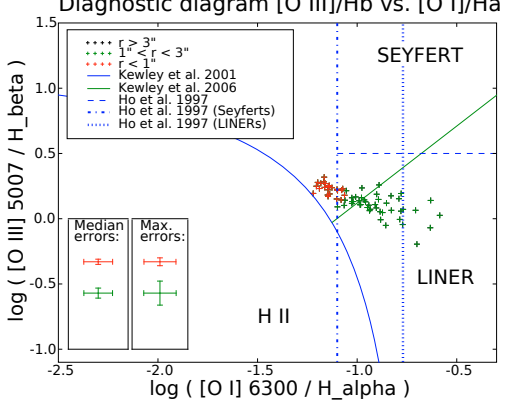

Fig. 4. Mrk 622. 

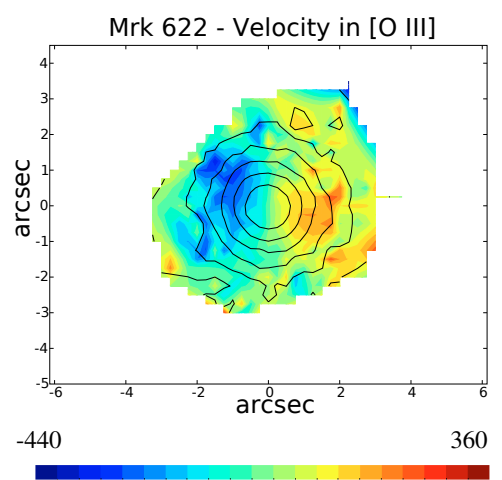

Mrk 622 - FWHM of [O III]
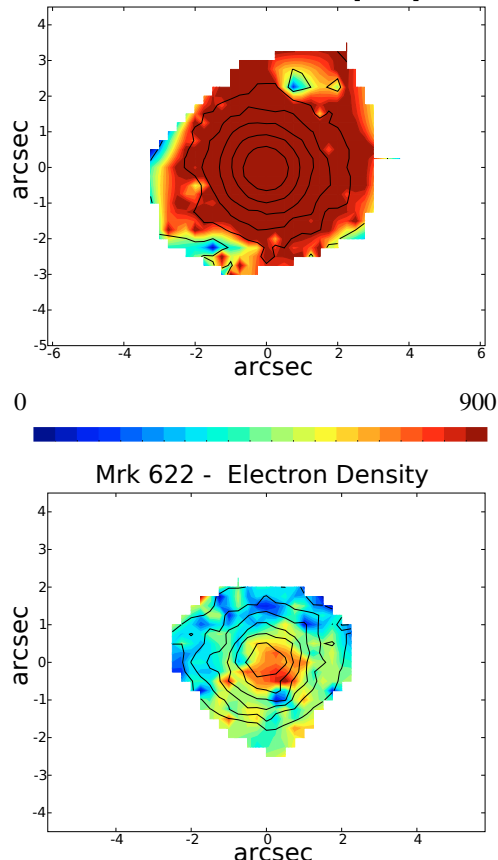

100

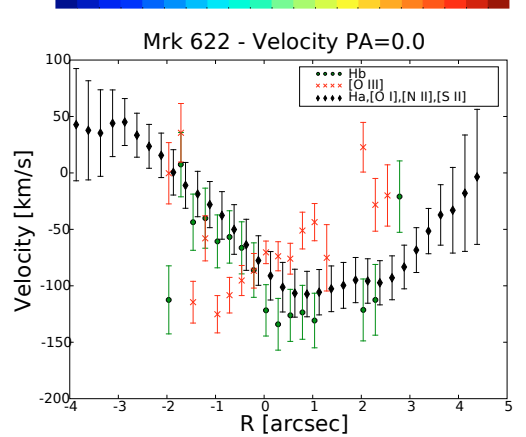

Mrk 622 - Diagnostic ratio [N II] $6583 / \mathrm{Ha}$

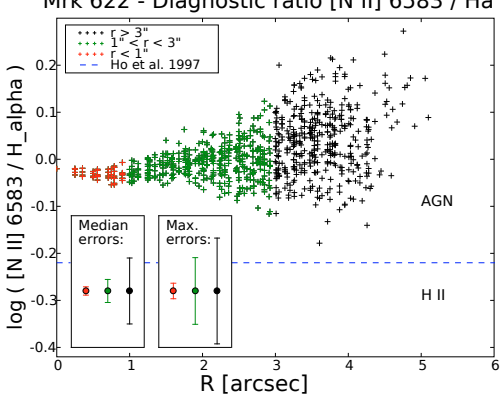

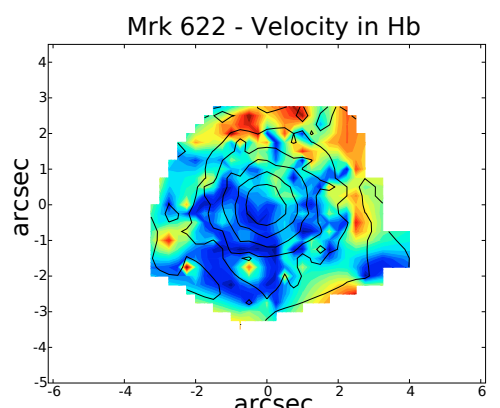
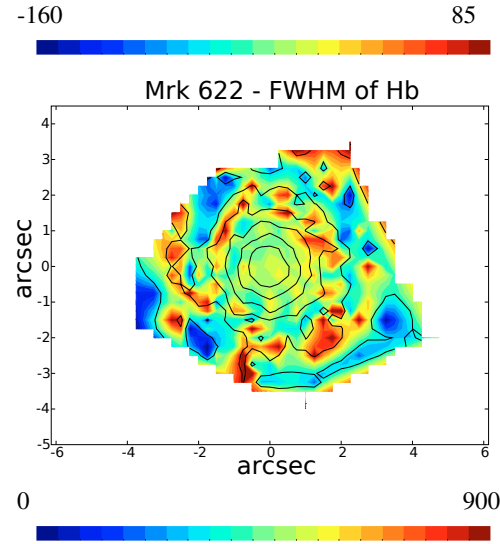

SSP mass Young/Total, $\mathrm{S} / \mathrm{N}=50$
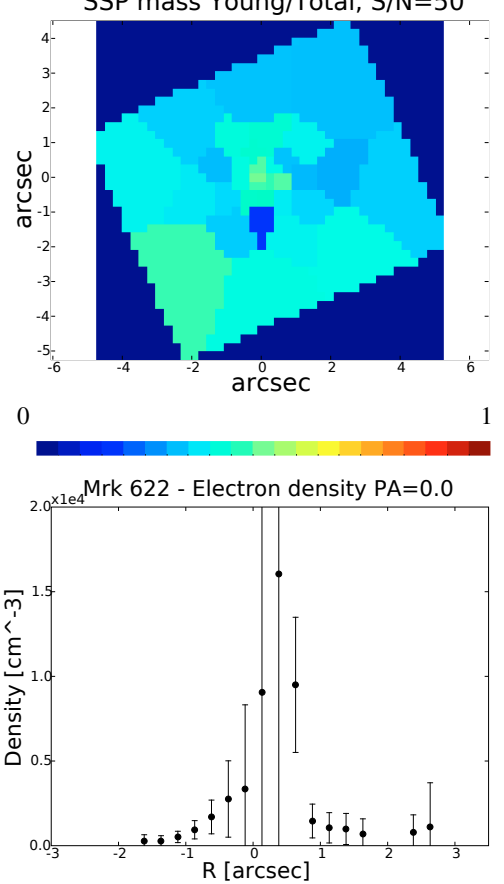

Mrk 622 - Diagnostic ratio [S II] $6717,6731 / \mathrm{Ha}$

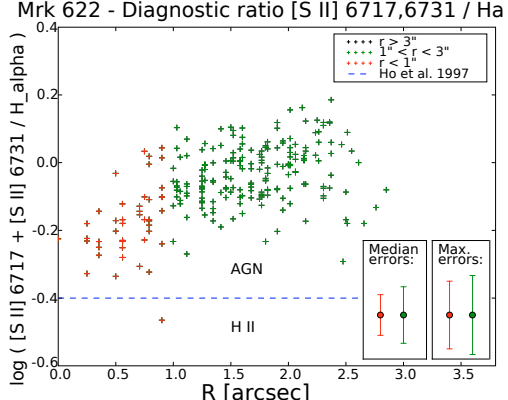

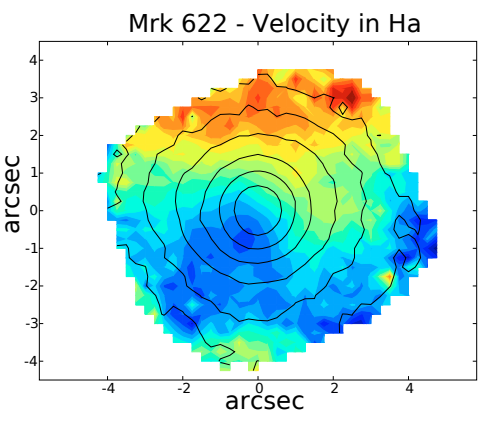

$-160$

85
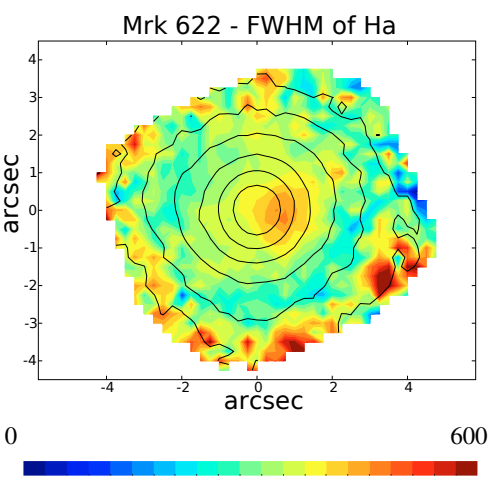

Stellar velocities $\mathrm{S} / \mathrm{N}=50$

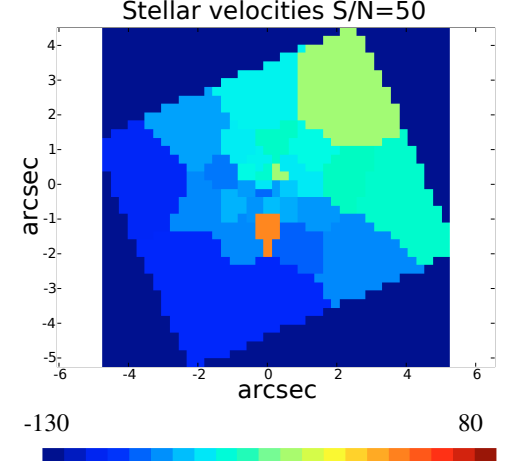

Mrk 622 - Diagnostic ratio [O III] 5007 / Hb

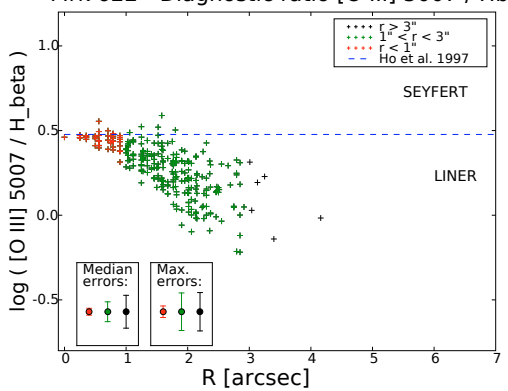

Mrk 622 - Diagnostic ratio [O I] $6300 / \mathrm{Ha}$

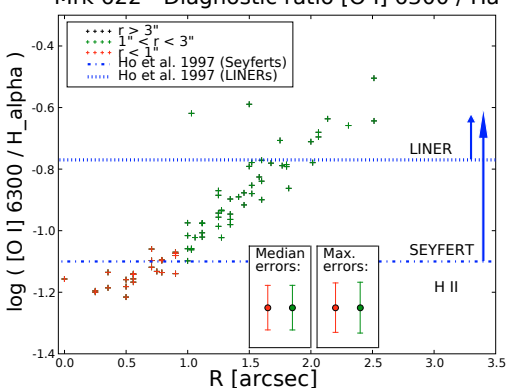

Fig. 5. Mrk 622. 
of the line profile is always biased towards the stronger component. As a result, our velocity map in [O III] is not a good approximation of the true motion and a detailed multi-component modelling of line profiles is necessary. We assume that this is the major reason for the dissimilarities between the hydrogen and $[\mathrm{O} \mathrm{III}]$ kinematics presented in Fig. 5. While the apparent kinematic axis of [O III] lies along $\mathrm{PA} \sim 90^{\circ}$, that of $\mathrm{H} \alpha$ (together with the low-ionisation lines) is perpendicular to it, at $\mathrm{PA} \sim 180^{\circ}$, measured in the outer parts of the $\mathrm{H} \alpha$ map, the central regions being characterised by S-shape isovelocity contours. The stellar velocity field, as far as can be deduced from our data, has a kinematic axis at PA $\sim 120^{\circ}$, and it is impossible to decide whether the twisted contours are present due to the low data quality.

The $\mathrm{H} \beta$ data are of low quality and their derived velocities do not agree with those measured from $\mathrm{H} \alpha$. We note that velocity colour scales in Fig. 5 were not homogenised to the same limits, due to the problematic fitting results. Two-component line profile modelling is necessary for [O III] lines, as the ambiguous interpretation of present results demonstrates.

The electron density, the interstellar reddening, and the gas excitation all reach their maximum values at the galactic centre. In contrast, the diagnostic ratios of line intensities, except for $[\mathrm{O} \mathrm{III}] / \mathrm{H} \beta$, reach their minima in the central region. Interestingly, the spatially resolved diagnostic diagrams indicate low excitation across the entire FOV (Fig. 4). The observed spectra possess strong $\mathrm{H} \beta$ absorption wings, which affect both the selection of the stellar population model and the predicted absorption in $\mathrm{H} \beta$, which may be overestimated.

\section{Mrk 1066}

While being classified as a marginal Seyfert by Osterbrock \& Dahari (1983), a high-excitation component of the spectral lines was found by Bower et al. (1995). Radio emission was observed at PA $\sim 135^{\circ}$ by Haniff et al. (1988); and Ulvestad \& Wilson (1989), and the narrow-band images indicated an alignment of the optical [O III] emission with the radio jet (Bower et al. 1995; Mulchaey et al. 1996a). The direction of both types of emission then agrees approximately with the orientation of the galactic bar of PA 143 (Mazzarella \& Boroson 1993; Bower et al. 1995). According to Bower et al. (1995), the velocities in $\mathrm{H} \alpha+[\mathrm{N} \mathrm{II}]$ are consistent with rotation, probably in the galactic disc, while [O III] shows little or no rotation, which they interpret as an outflow. No signs of hidden BLR were inferred from spectropolarimetry (Miller \& Goodrich 1990). No central dust lane in high-resolution HST images, nor other evidence of a torus (such as excess reddening at the centre) was found by Bower et al. (1995). Zhang \& Wang (2006) attributed the non-detection of a hidden BLR to orientation effects and/or the low mass of the central black hole.

The emission that we observe in all the lines is slightly extended along $\mathrm{PA} \sim 130^{\circ}-140^{\circ}$, i.e., parallel to the galactic bar. A prominent emission feature located in the east is seen in the hydrogen lines and partially in [N II] and [S II], while absent in [O III], and is associated with high interstellar reddening, low excitation, and a small velocity dispersion (Figs. 7 and 8). We conclude that it is probably a region that has not been ionised by the AGN. Excitation is highest in the central parts and extends asymmetrically to the north-west side along the major axis of [O III] emission, as well as along the minor axis to both sides from the centre. Interestingly, the interstellar reddening extends asymmetrically along the major axis in the opposite direction, to the south-east (Fig. 7). The maps of diagnostic ratios
$[\mathrm{O} \mathrm{I}] / \mathrm{H} \alpha$ and $[\mathrm{N} \mathrm{II}] / \mathrm{H} \alpha$ exhibit surprising distributions of high values within an extended region along the minor axis (Fig. 7). The diagnostic diagrams are consistent with the interpretation of the object as a marginal Seyfert (Fig. 7). With a few exceptions, all of the spectra are characterised by an $[\mathrm{O} \mathrm{III}] / \mathrm{H} \beta$ intensity ratio that is below the empirical Seyfert limit, including the central parts. However, a proper disentangling of the asymmetric lines may confirm the high-excitation component (Bower et al. 1995). The electron density reaches peak in the central region, the maximum being off-centred by $\sim 200$ pc (Figs. 8 and 9).

The $\mathrm{H} \alpha$ velocity map (Fig. 8) displays a clear S-shaped pattern. Velocities in [O III] exhibit important differences from the hydrogen data, seen mainly in the one-dimensional cuts. We found no multi-component line profiles for this object (Fig. 9), even though we observe a large- $F W H M$ region extending along the minor axis, which is usually associated with line splits in other objects of the sample. On the other hand, most lines have blue wings across the entire FOV.

\section{NGC 262 (Mrk 348)}

The host-galaxy is orientated almost face-on and has a close companion, NGC 266. The nuclear region has been a popular target of observations across the entire electromagnetic spectrum, because of the difficulties to interpret orientations of individual components observed in the different spectral regions within a simple model (Antón et al. 2002). Capetti et al. (1996) reported a prominent central dust lane of scale height $50 \mathrm{pc}$, perpendicular to the optical [O III] emission in an HST image. Evidence of a torus or related structure was found in IR images by Simpson et al. (1996). A polarised broad $\mathrm{H} \alpha$ component (Miller \& Goodrich 1990) and a hard X-ray emission (Awaki et al. 1991) inferred a hidden Seyfert 1 nucleus. The radio emission is in the form of a triple radio source of spatial extent $0.2^{\prime \prime}$ along PA $=168^{\circ}$ (Neff \& de Bruyn 1983), variable on the scale of months. Megamaser emission detected in the central few milliarcseconds (Falcke et al. 2000; Xanthopoulos \& Richards 2001; Peck et al. 2003) was interpreted as an interaction between the radio jet (situated close to the plane of the sky) and a molecular cloud. Optical [O III] emission is well aligned with the radio on sub-arcsecond scales (Falcke et al. 1998; Schmitt et al. 2003a), while on the arcsecond scales it is extended along PA $\sim 185^{\circ}$ (Schmitt et al. 2003a), with a secondary blob of emission $\sim 0.9^{\prime \prime}$ south of the nucleus. A hint of ionisation cones was found by Simpson et al. (1996) and Mulchaey et al. (1996a) in the ionisation map, but the evidence is marginal according to Antón et al. (2002). NIR emission map obtained with an IFU by Sosa-Brito et al. (2001) does not have any prominent direction on the scale of $3^{\prime \prime}$, although contours in the inner parts seem to have a slight directionality along $\mathrm{PA} \sim 10^{\circ}$ and $\mathrm{PA} \sim 90^{\circ}$. A ring of $\mathrm{H}$ II emission on the scale of $\sim 1 \mathrm{kpc}$ was reported by Antón et al. (2002) and Mulchaey et al. (1996a). The galaxy possesses a giant $\mathrm{H}$ I envelope on the scales of hundreds of kpc (Heckman et al. 1982).

The face-on orientation of the galaxy is confirmed in our data by the low stellar LOS velocities (Fig. 11), the minimum and maximum values that we obtained from our fits are separated by $70 \mathrm{~km} \mathrm{~s}^{-1}$ only. We estimate the kinematic stellar axis to be at PA $\sim 70^{\circ}$. This Seyfert galaxy has one of the lowest contributions from young stars of the entire sample. Mass fractions of young stars above several percent have been identified in the central $\sim 250$ pc only.

The emission maps of most of the emission lines are slightly extended along $\mathrm{PA} \sim 10^{\circ}$ (Fig. 10), which is consistent with 

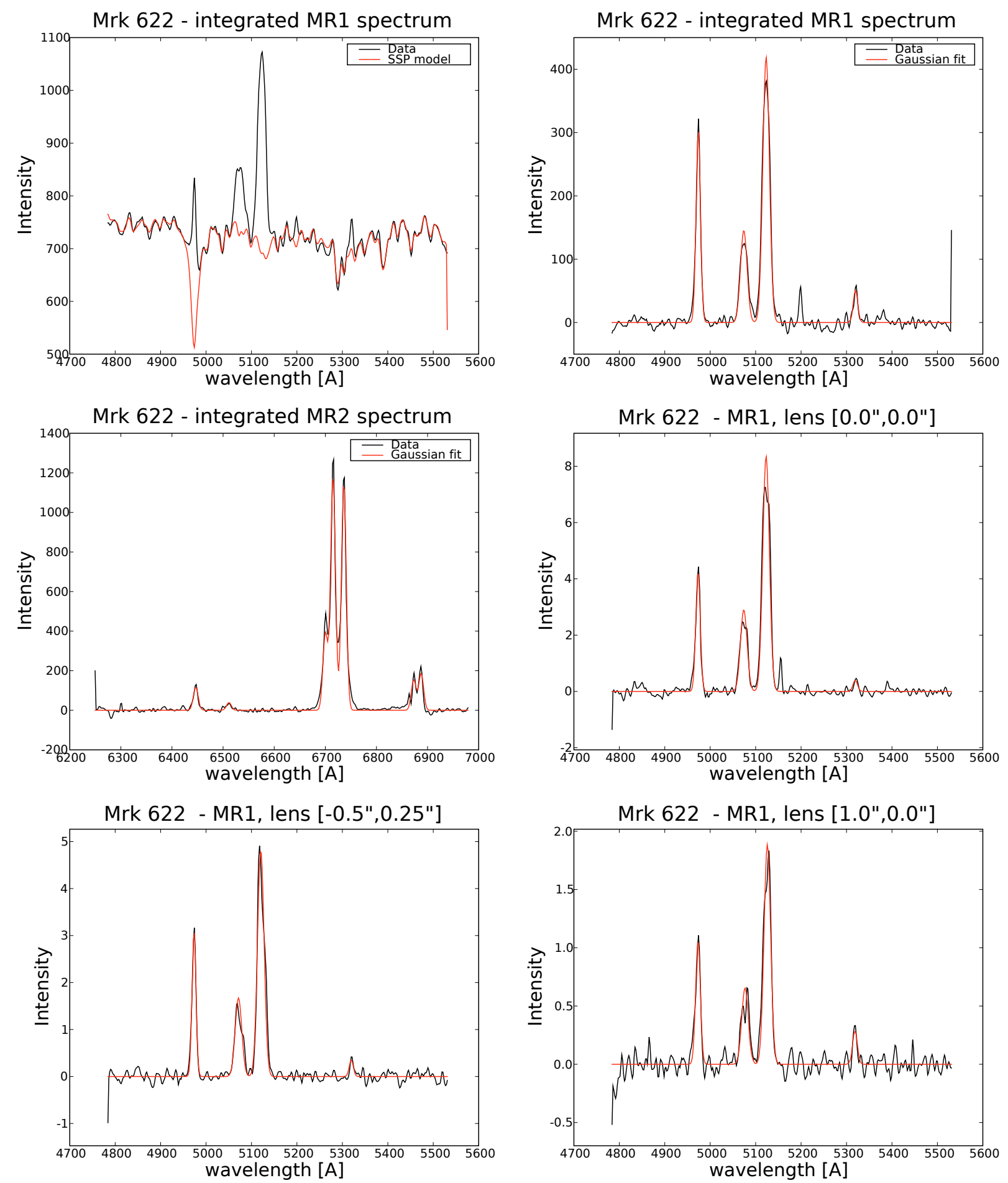

Fig. 6. Mrk 622.

Sosa-Brito et al. (2001), and differs by approximately $20^{\circ}$ from the radio jet reported in the literature. We partially resolve the secondary emission maximum known from the literature, $\sim 1^{\prime \prime}$ south-west of the centre. The gas excitation is high, and all the regions are high in the Seyfert regime according to the spatially resolved diagnostic diagram (Fig. 10). The regions of highest excitation are situated along the major axis of emission, within a $\mathrm{PA}$ range of $\sim 5^{\circ}-30^{\circ}$. The $[\mathrm{O} \mathrm{III}] / \mathrm{H} \beta$ ratio decreases steeply with radial distance, as seen in the corresponding graph of Fig. 11. We note that the ratios of low-ionisation lines to $\mathrm{H} \alpha$ show prominent minima south-west of the galactic centre, in the region of the receding velocity maximum (see paragraph below). In addition, $[\mathrm{S} \mathrm{II}] / \mathrm{H} \alpha$ has a minimum at the centre, surrounded by a patchy ring of high $[\mathrm{S} \mathrm{II}] / \mathrm{H} \alpha$ on scales of $\sim 600 \mathrm{pc}$, which corresponds to a ring of low electron densities derived from the [S II] ratio (Figs. 10 and 11).

The gas velocities (Fig. 11) are the most surprising detection. We observe two spots of velocity maxima of opposite sign, aligned at $\mathrm{PA} \sim 25^{\circ}$, i.e., approximately parallel to the major axis of optical emission. The two spots of $>100 \mathrm{~km} \mathrm{~s}^{-1}$, present 


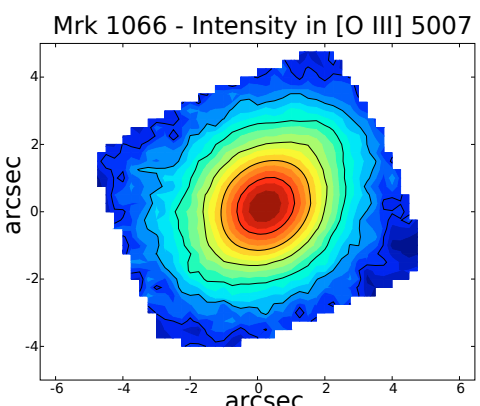

1.5

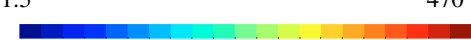

Mrk 1066 - Ratio [O III] 5007 / Hb

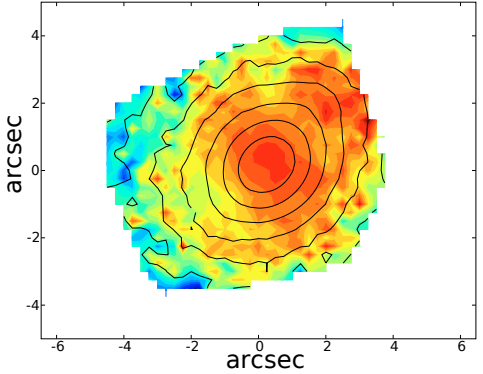

0.4

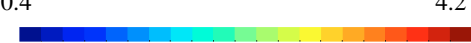

Mrk 1066 - Intensity in [O I] 6300

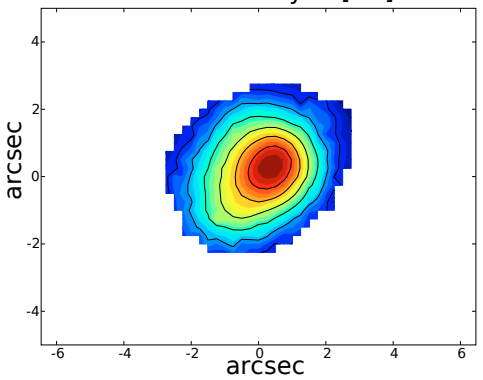

1.5

110

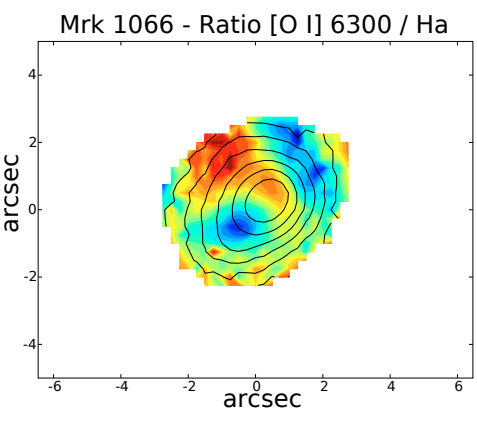

0.05

0.2

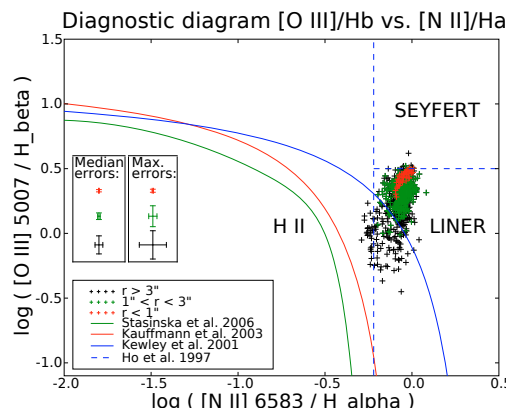

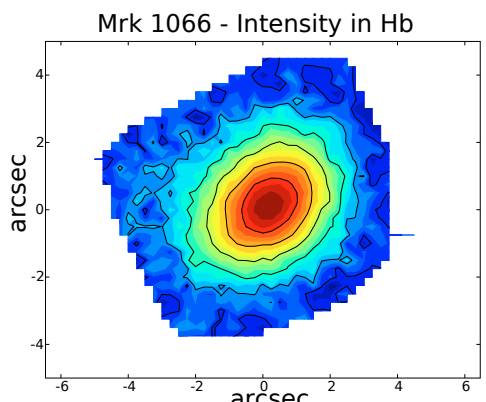

1.5

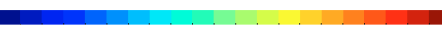

Mrk 1066 - Intensity in [N I] 5200

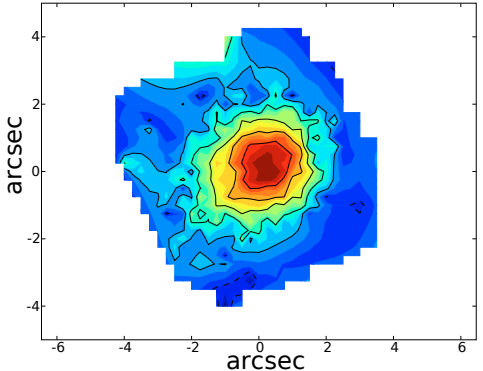

0.05

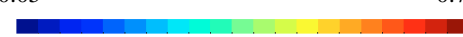

Mrk 1066 - Intensity in [N II] 6583

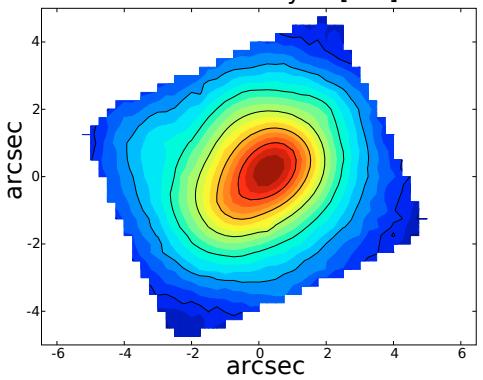

0.2
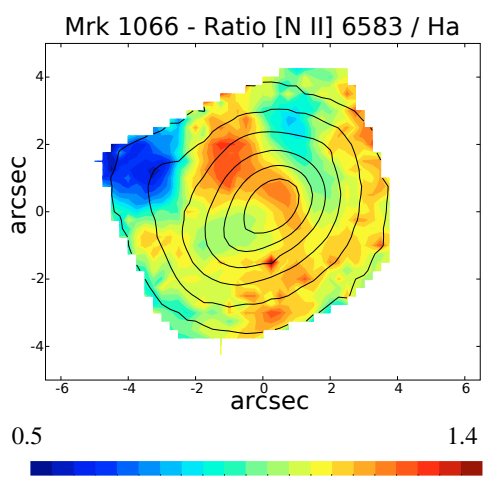

Diagnostic diagram [O III]/Hb vs. [S II]/Ha

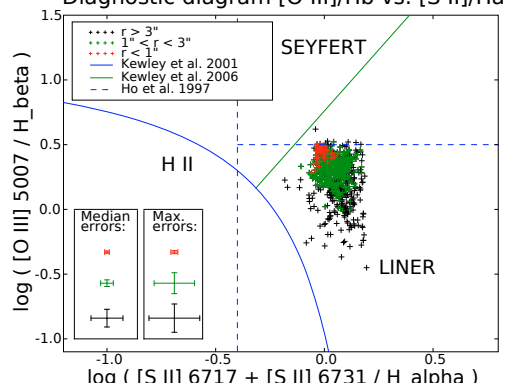

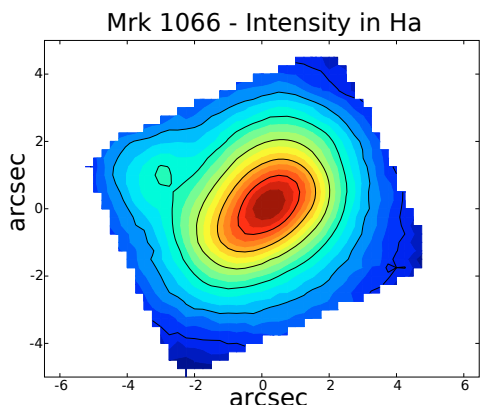

2

1300

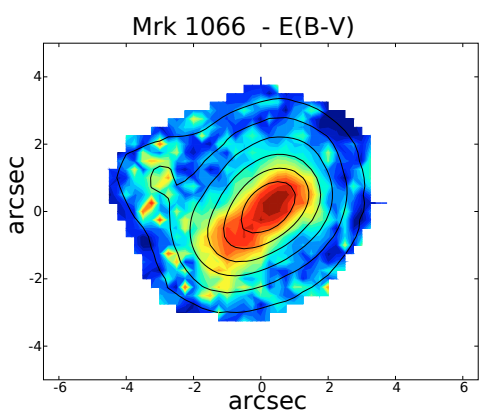

0

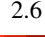

Mrk 1066 - Intensity in [S II] 6717,6731

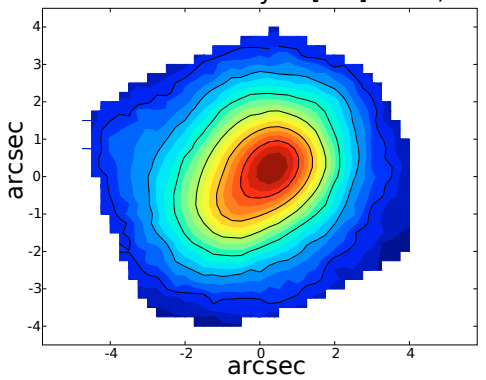

2.5

540

Mrk 1066 - Ratio [S II] 6717,6731 / Ha
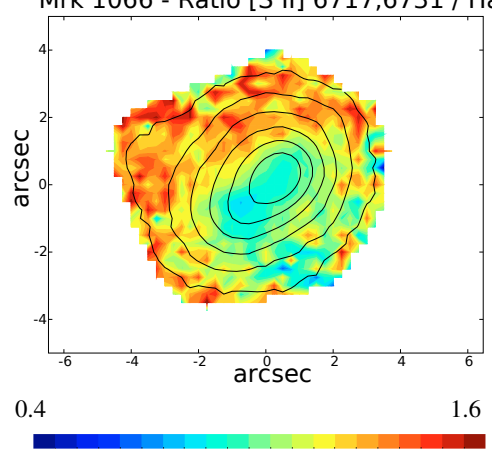

Diagnostic diagram [O III]/Hb vs. [O I] $/ \mathrm{Ha}$

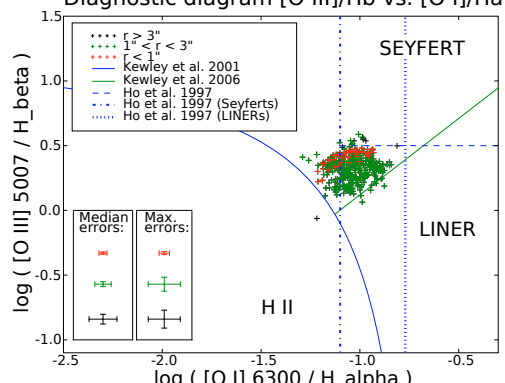

Fig. 7. Mrk 1066. 

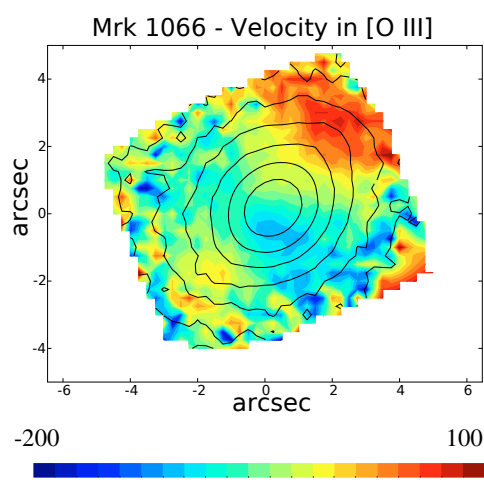

Mrk 1066 - FWHM of [O III]

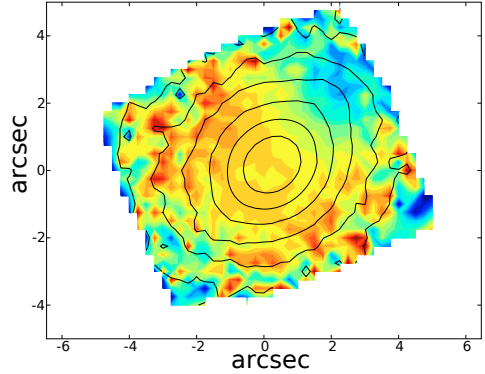

0

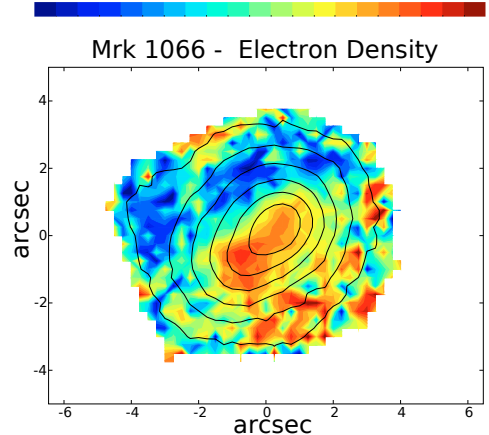

100

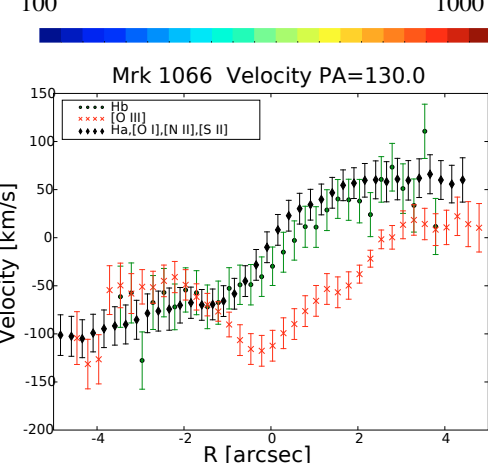

Mrk 1066 - Diagnostic ratio [N II] 6583 / Ha

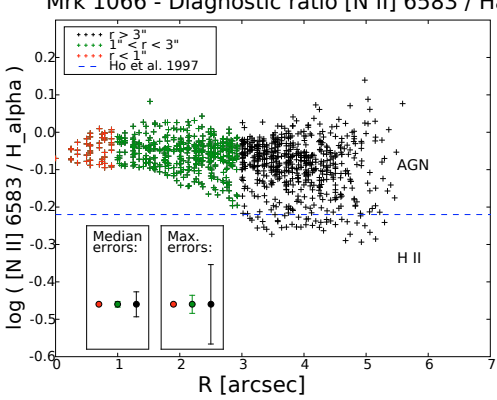

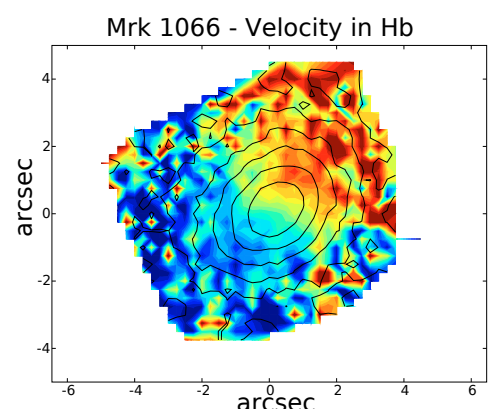

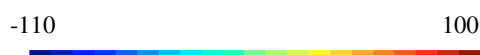

Mrk 1066 - FWHM of $\mathrm{Hb}$

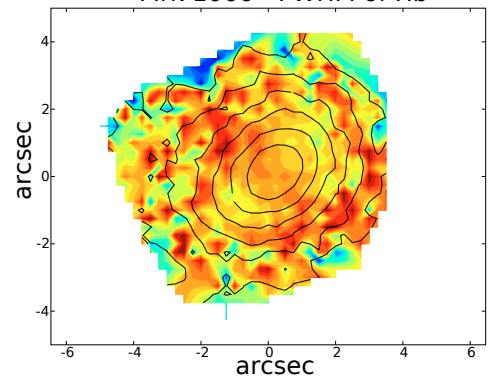

700

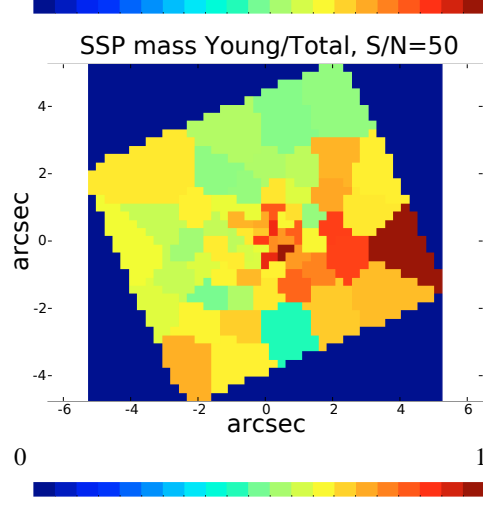

Mrk 1066 - Electron density PA $=130.0$

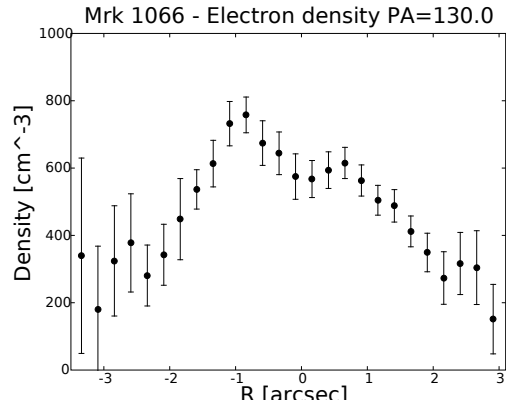

Mrk 1066 - Diagnostic ratio [S II] 6717,6731 / Ha

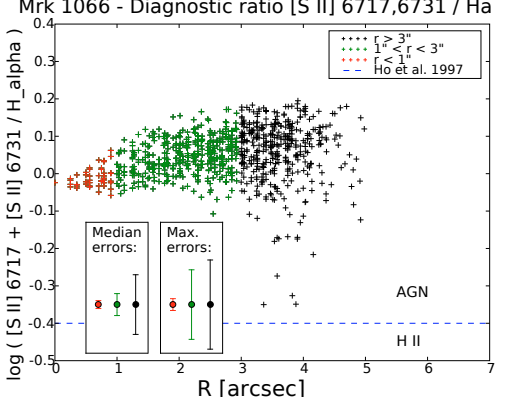

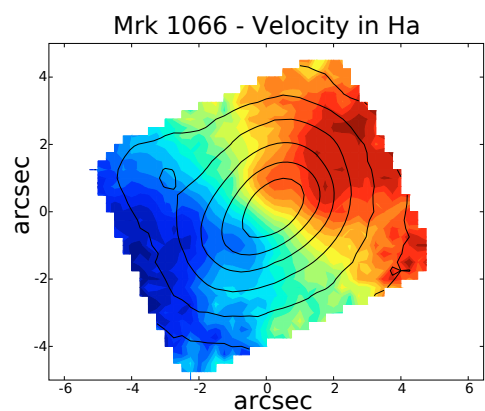

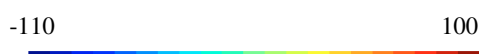

Mrk 1066 - FWHM of Ha

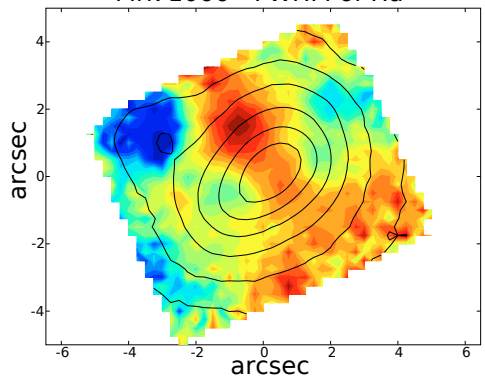

400

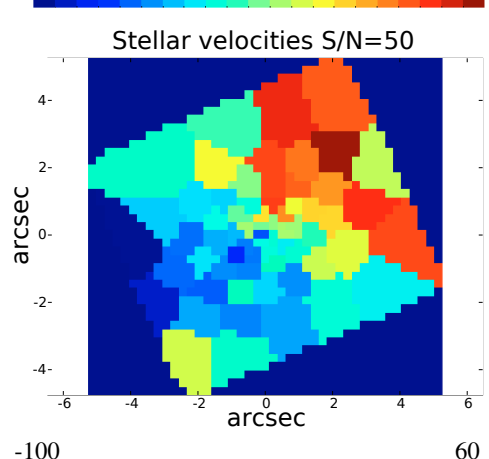

Mrk 1066 - Diagnostic ratio [O III] 5007 / Hb

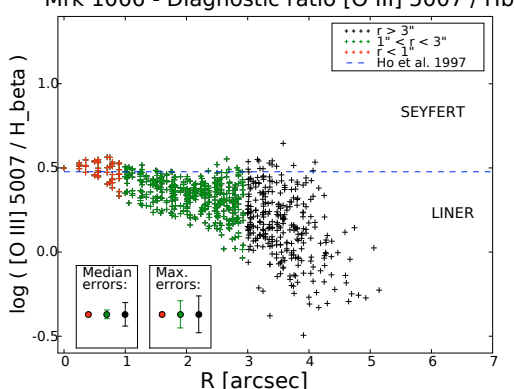

Mrk 1066 - Diagnostic ratio [O I] $6300 / \mathrm{Ha}$

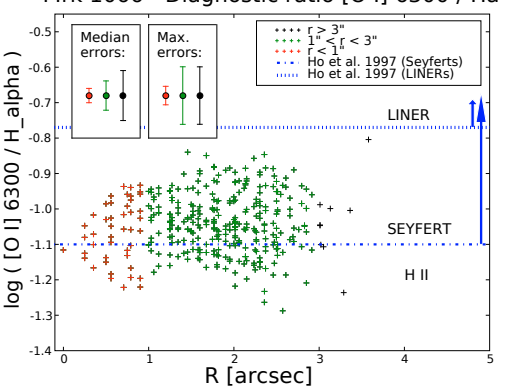

Fig. 8. Mrk 1066. 

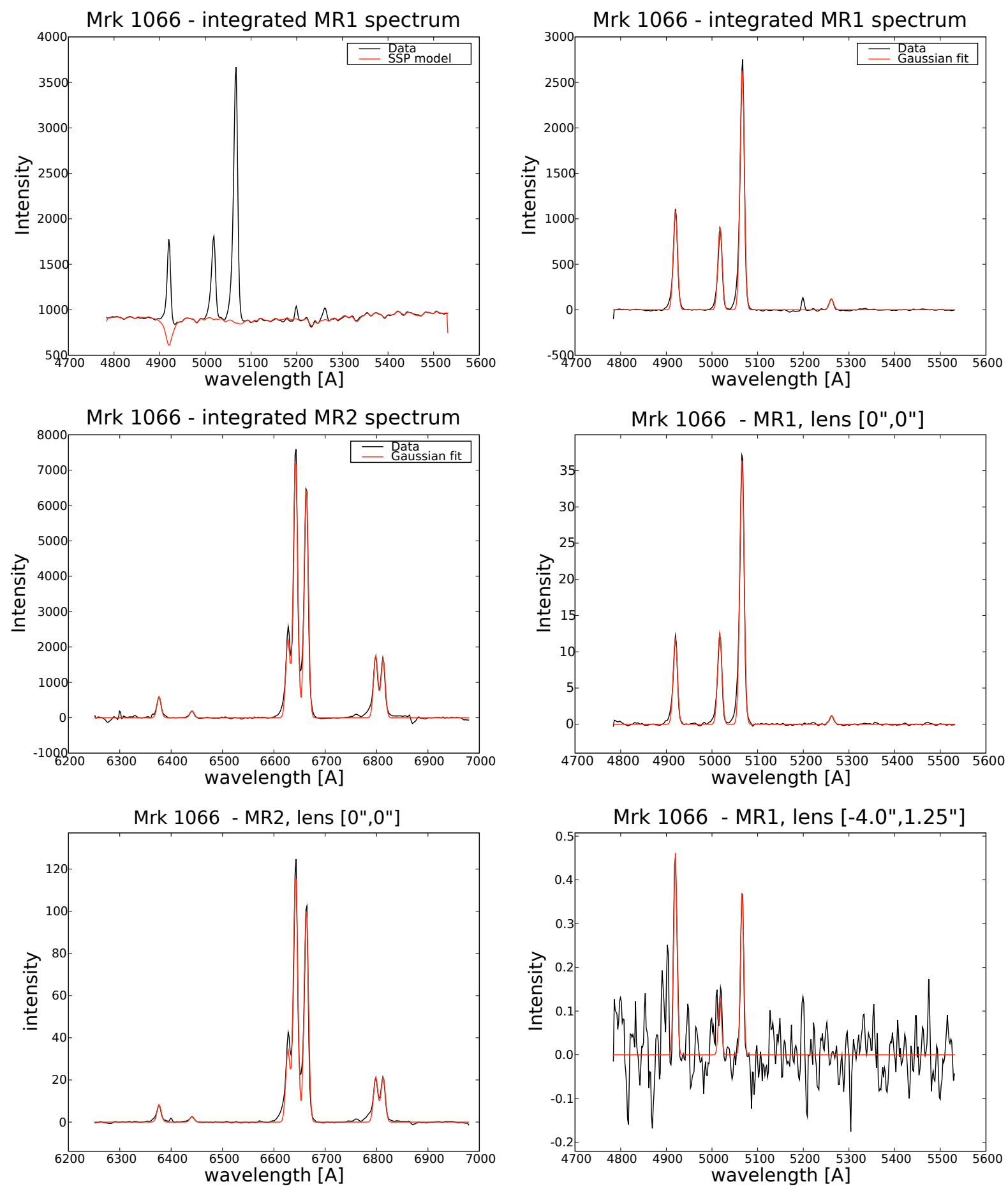

Fig. 9. Mrk 1066.

in [O III] as well as in low-ionisation lines, about $200 \mathrm{pc}$ in size, are placed symmetrically $\sim 300 \mathrm{pc}$ from the emission maximum. We assume that the observation corresponds to a rotating ring, inclined with respect to the galactic disc. However, there is a clear asymmetry in the radial profile of the two velocity peaks (Fig. 11).

The maxima of the LOS velocity dispersion in $\mathrm{H} \alpha$ are located at the positions of the velocity peaks, which is not the case for [O III]. The low-ionisation emission lines also have significantly asymmetric profiles across the FOV, unlike [O III] where mostly blue wings are present (Fig. 12). The low-ionisation lines are characterised by prominent blueshifted and redshifted components shifted by $300-400 \mathrm{~km} \mathrm{~s}^{-1}$ from the main component. The red asymmetries were found mainly in the southern part of the FOV. The vicinity of the south-western spot of maximal velocities is characterised by rapid spatial variations in the line shapes and switching between red and blue additional components. A careful disentangling of the line profiles is necessary before further conclusions can be made.

We detect another systematic motion in [O III], at the edge of the FOV, of radii $\sim 1 \mathrm{kpc}$, where we observe velocities with a polarity that is reversed with respect to the nuclear ring. We verified 


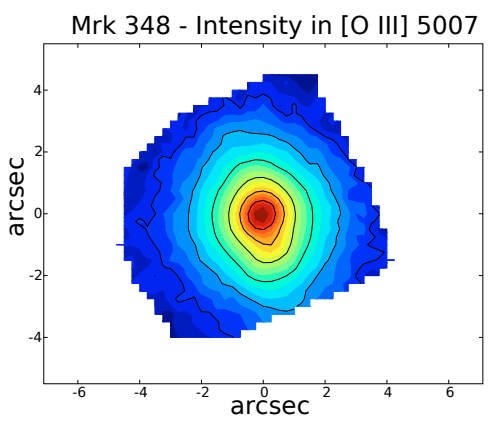

1.5

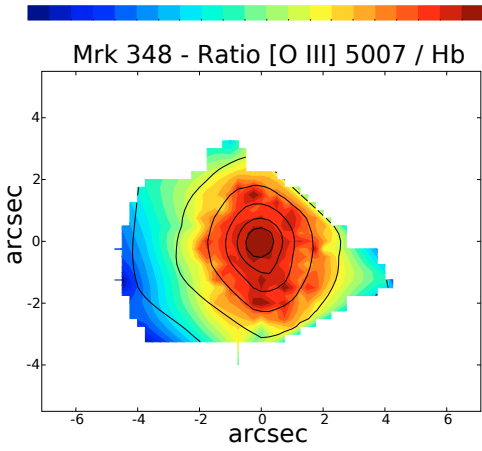

0.5
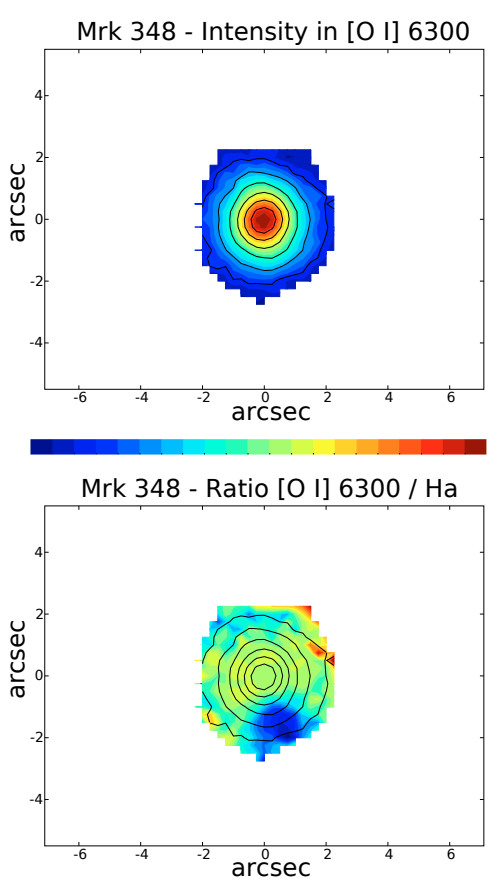

0.2

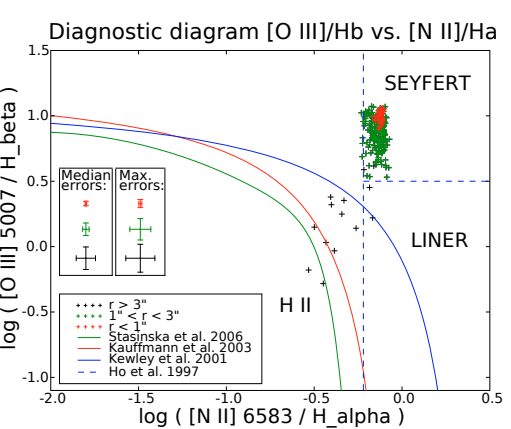

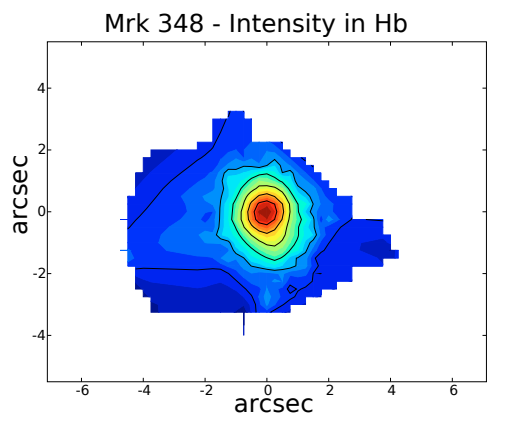

1.6
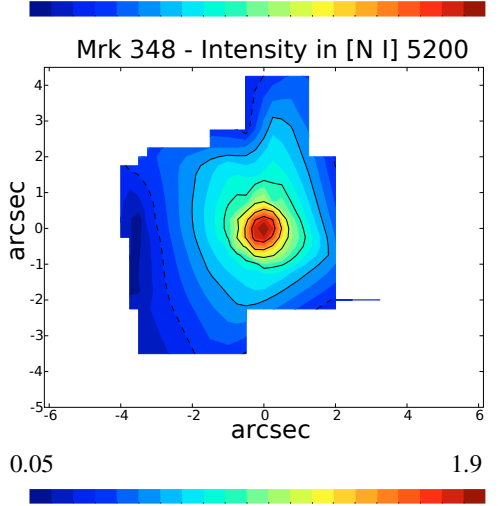

Mrk 348 - Intensity in [N II] 6583

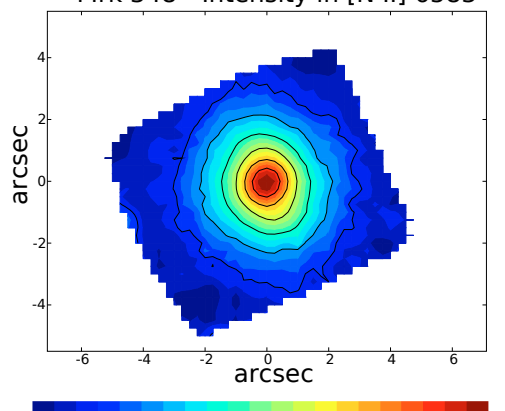

Mrk 348 - Ratio [N II] 6583 / Ha

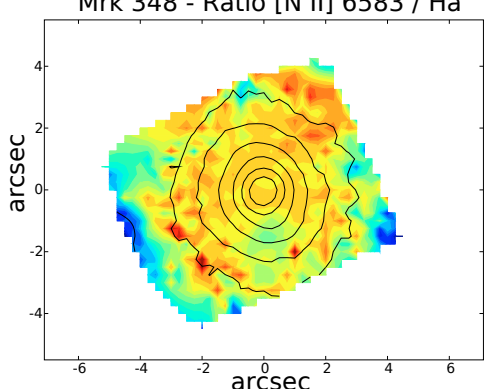

0.3

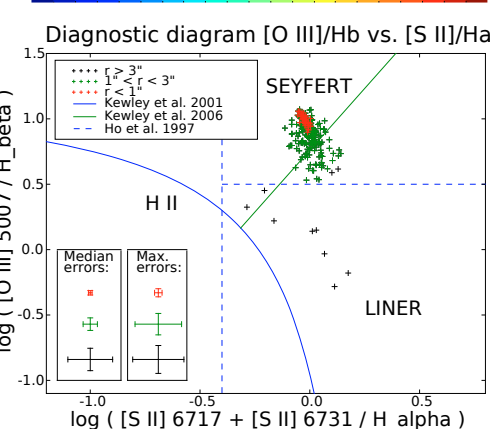

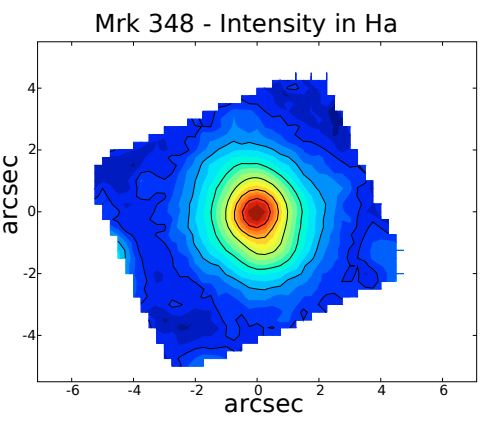

1.3 1240

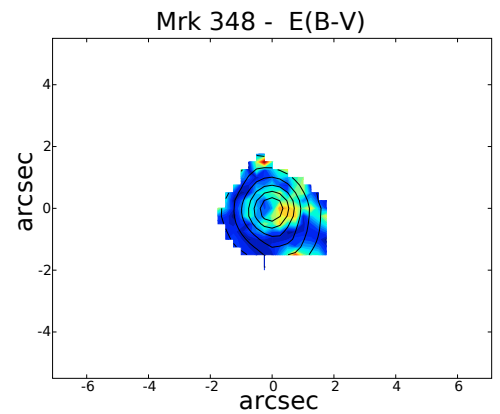

0
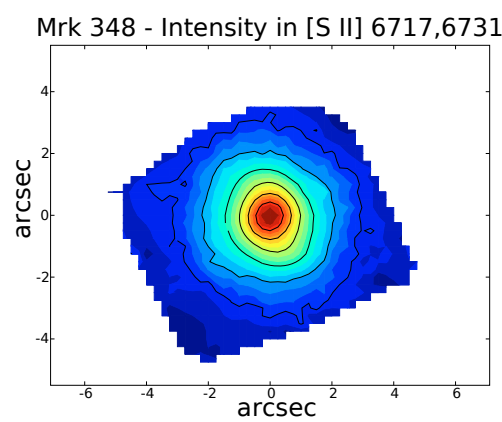

Mrk 348 - Ratio [S II] 6717,6731 / Ha

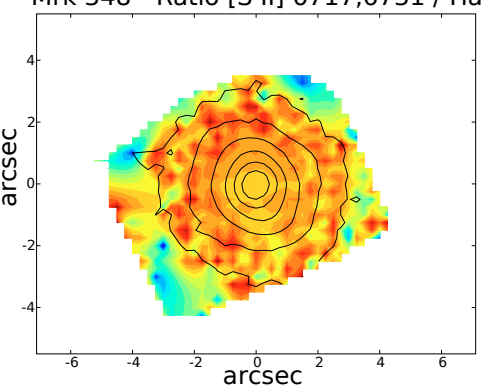

0.3

1.5

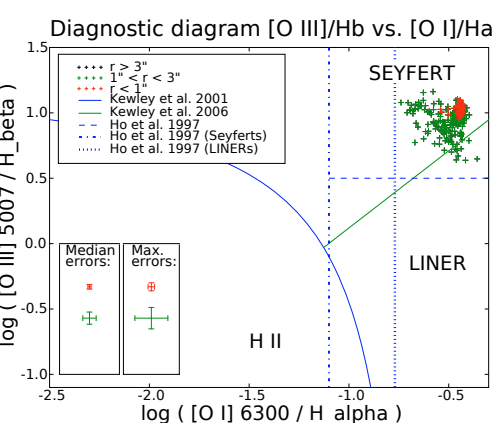

Fig. 10. NGC 262 (Mrk 348). 


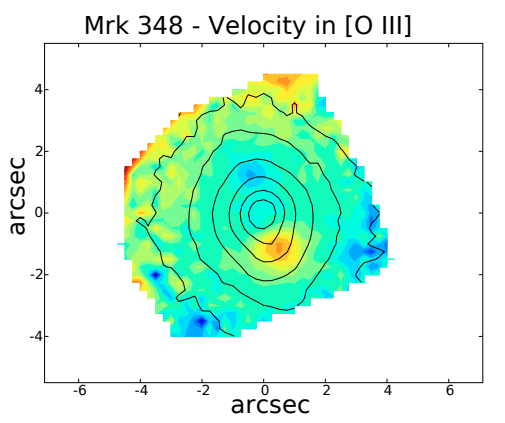

$-180$
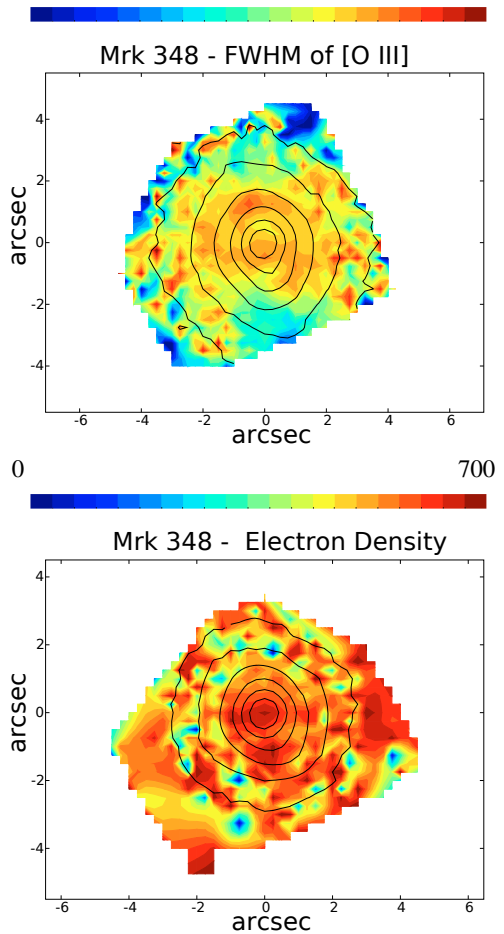

100
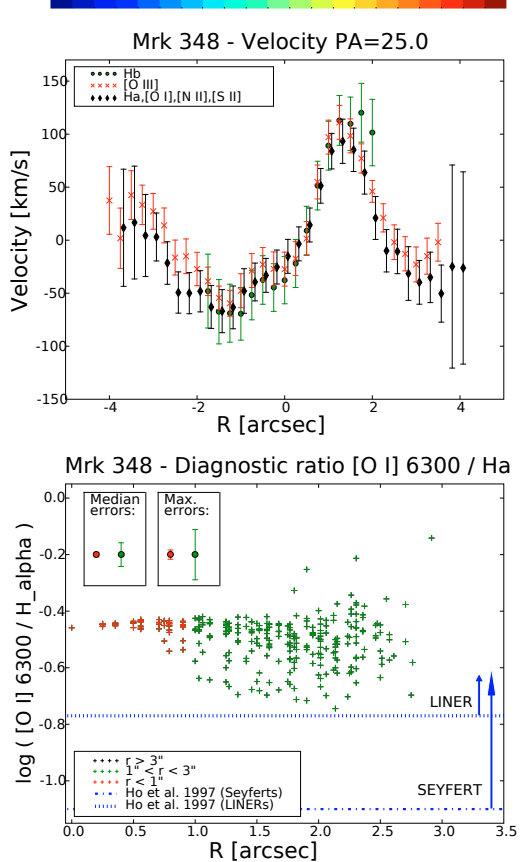

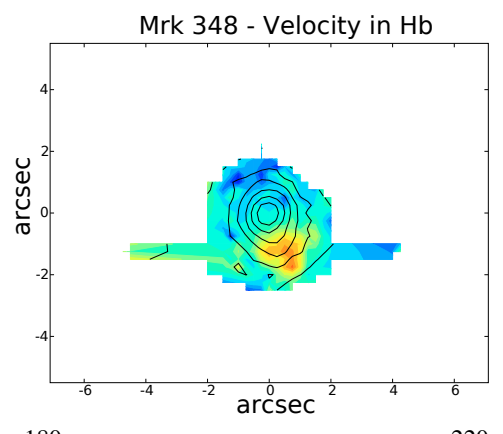

$-180$

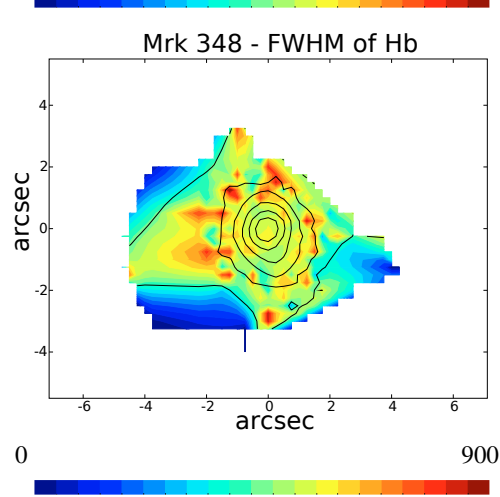

SSP mass Young/Total, $\mathrm{S} / \mathrm{N}=50$

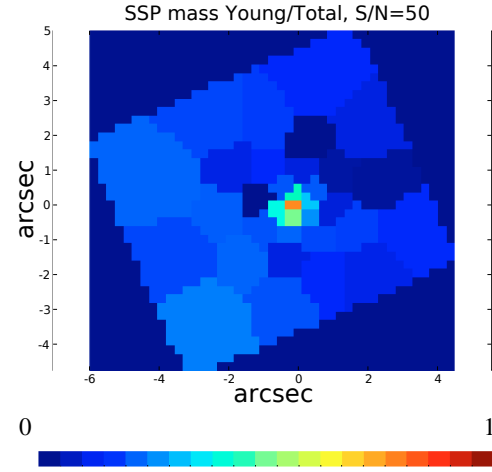

Mrk 348- Electron density $\mathrm{PA}=25.0$

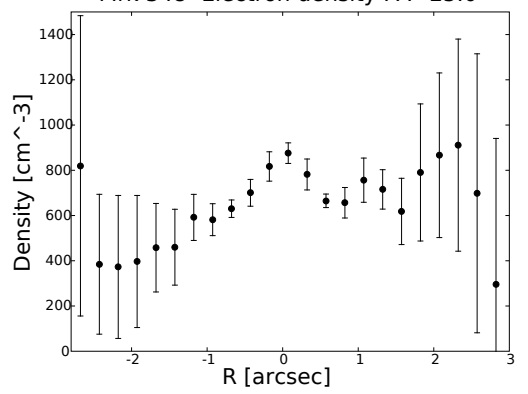

Mrk 348 - Diagnostic ratio [N II] $6583 / \mathrm{Ha}$

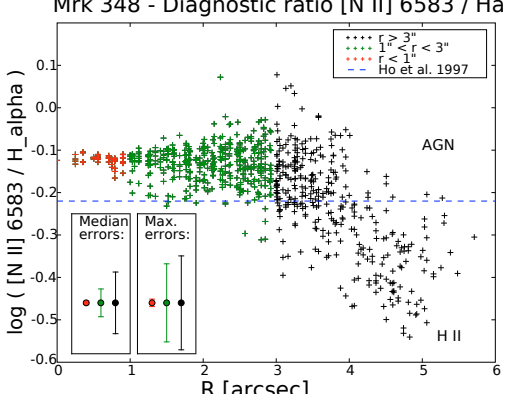

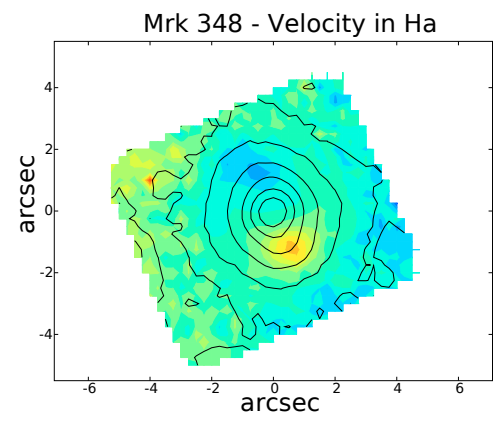

$-180$ 220
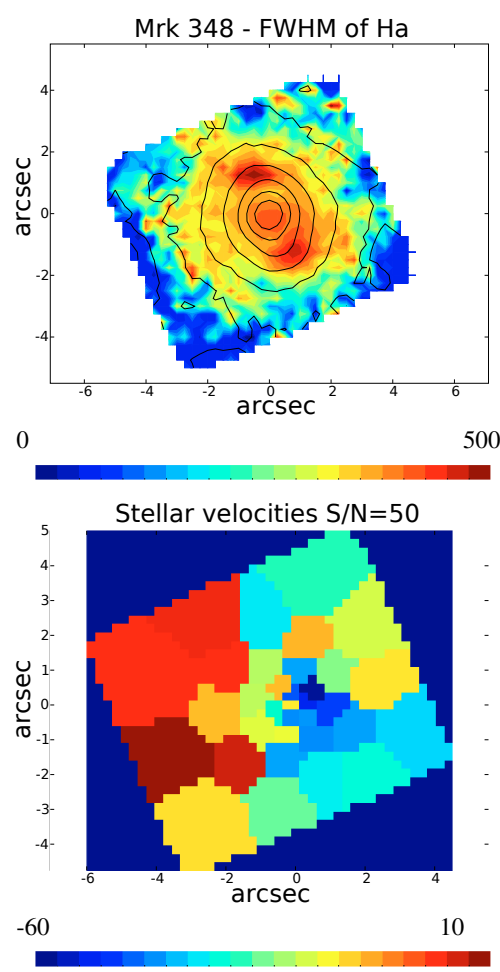

Mrk 348 - Diagnostic ratio [O III] 5007 / Hb

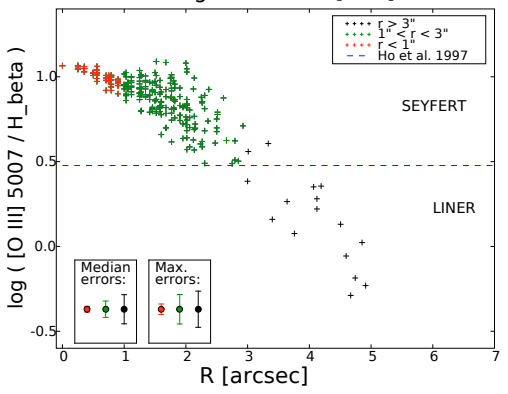

Mrk 348 - Diagnostic ratio [S II] 6717,6731/ Ha

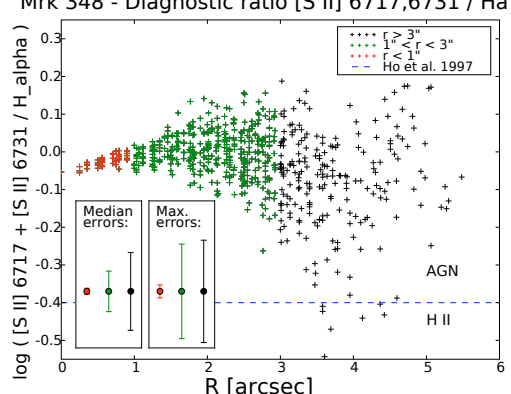

Fig. 11. NGC 262 (Mrk 348). 
that it is not the effect of noise and suggest that it might correspond to the ring identified by Antón et al. (2002) and Mulchaey et al. (1996a).

\section{NGC 449 (Mrk 1)}

The galaxy is well known to possess a prominent water maser in its nucleus (Braatz et al. 1994). No evidence of a hidden Seyfert 1 nucleus was found, in either polarised light (Kay 1994) or in the infrared (Veilleux et al. 1997). Mulchaey et al. (1996a) reported [O III] emission extending to $\sim 3 \mathrm{kpc}$, co-aligned with both the galaxy major axis, $\mathrm{PA}=83^{\circ}$ and $20 \mathrm{~cm}$ radio emission on the scale of $\sim 30$ pc (Kukula et al. 1999). Stellar-population synthesis modelling of long-slit data of $3 \AA$ resolution performed by Raimann et al. (2003) revealed mostly intermediate-age stellar populations $(\sim 1 \mathrm{Gyr})$ in the central kpc and an increasingly dominant old population ( $\sim 10 \mathrm{Gyr})$ outside this radius.

Our OASIS data for this object were obtained as a mosaic of three partially overlapping fields. We confirm strongly collimated emission in [O III], H II, and [N II] extending to radii of at least $\sim 3 \mathrm{kpc}$ (Fig. 13). In the other observed lines, the $S / N$ is insufficient to obtain reliable data to these distances. We found a misalignment between the emission in [O III] $\left(\mathrm{PA} \sim 65^{\circ}\right)$ and the low-ionisation species $\left(\mathrm{PA} \sim 85^{\circ}\right.$ ). The velocity map in $\mathrm{H} \alpha$ suggests circular rotation. As in most of the targets of the OASIS sample, we found a region of large $F W H M$, stretching along the minor axis (Fig. 14). The region is characterised by multi-component line profiles, usually in the form of red or blue wings in [O III] and split line profiles in low-ionisation species (Fig. 15). Outside the central $\sim 600 \mathrm{pc}$, the emission lines are narrow.

Our stellar data were not of a high quality for this object. The maximum contribution of young stars was fitted in the nuclear region. The map of stellar velocities could not be interpreted reliably.

\section{NGC 2273 (Mrk 620)}

NGC 2273 is a barred galaxy (van Driel \& Buta 1991), with a star-forming nuclear ring (Ferruit et al. 2000). Deviations from axial symmetry were confirmed by GMOS IFU observation of the stellar kinematics in the central regions (Barbosa et al. 2006). Radio emission extends along PA $\sim 20^{\circ}$ at the $20 \mathrm{~cm}$ wavelength, and two components along PA $\sim 90^{\circ}$ are evident at the $6 \mathrm{~cm}$ wavelength in the central 1" (Ulvestad \& Wilson 1984b; Ho \& Ulvestad 2001). HST observations resolved a jet-like [O III] structure extending $2^{\prime \prime}$ east of the nucleus, at PA $\sim 90^{\circ}$, aligned with the double radio source (Ferruit et al. 2000). Mulchaey et al. (1996a) reported extended [O III] emission reaching to $\sim 6.5^{\prime \prime}$ at $\mathrm{PA}=134^{\circ} \pm 10^{\circ}$. Moiseev et al. (2004) identified important differences between the velocities in [O III] and the Balmer lines.

Our data for this object are limited to a single spectral domain (MR1). While the [O III] emission does not exhibit any prominent axis (only a slight elongation is visible along $\left.\mathrm{PA} \sim 95^{\circ}\right), \mathrm{H} \beta$ emission seems to follow the direction of $\mathrm{PA} \sim 20^{\circ}$. These differences in behaviour are emphasised in the $[\mathrm{O}$ III $] / \mathrm{H} \beta$ map, where extended cone-like features appear along $\mathrm{PA} \sim 100^{\circ}$ (Fig. 16). The velocity field in [O III] lines shows S-shaped isocontours (Fig. 16), which might indicate deviations of the gravitational potential from axisymmetry. The data quality in $\mathrm{H} \beta$ is insufficient to confirm or exclude a similar velocity pattern in hydrogen. However, one-dimensional cuts of the velocity fields show important systematic differences between LOS velocities in [O III] and $\mathrm{H} \beta$. The stellar velocity field is well measured for this galaxy and shows rotation in a direction similar to gas, with the kinematic axis at the outer parts of the FOV approximately parallel to the $\mathrm{H} \beta$ photometric major axis. No asymmetric or multi-component emission-line profiles have been found, apart from weak, blue wings (Fig. 16).

\section{NGC 2992}

The host galaxy disc is highly inclined at $i \sim 70^{\circ}$, which makes it suitable for extraplanar emission studies. The galaxy has a companion NGC 2993, which is located $\sim 3^{\prime}$ south-east, connected with a tidal bridge, and whose tidal forces might have an important effect on the kinematics. Radio observations at $20 \mathrm{~cm}$ found a $25^{\prime \prime}$ structure along the major axis of the galaxy, and a $90^{\prime \prime}$ one-sided extension at PA $\sim 100^{\circ}-130^{\circ}$, close to the galaxy minor axis (Ward et al. 1980). The $6 \mathrm{~cm}$ radio emission is concentrated in the central parts, forming a "figure-eight" structure at $\mathrm{PA}=160^{\circ}($ Ulvestad \& Wilson $1984 \mathrm{~b}$; Wehrle \& Morris 1988). Soft X-rays are detected up to scales of $35^{\prime \prime}-45^{\prime \prime}$ along the galaxy's minor axis, and are co-spatial with the $20 \mathrm{~cm}$ radio emission. The [O III] emission forms two sharp-cut cones with a projected opening angle of $120^{\circ}$ (Márquez et al. 1998), their axes aligned with the galactic minor axis $\left(\mathrm{PA}=120^{\circ}\right)$ and approximately with the radio structure. A disturbed dust lane was identified at $\mathrm{PA}=15^{\circ}$ by Márquez et al. (1998) and at $\mathrm{PA}=30^{\circ}$ by Veilleux et al. (2001). Multi-component optical emission lines were detected, and the velocity field was suggested to be a superposition of rotation and an outflow (Márquez et al. 1998; Allen et al. 1999; Veilleux et al. 2001).

The FOV in OASIS observations enables only one of the emission cones to be imaged, located south-east of the dust lane. The emission structure that we observe (Fig. 17) is elongated at $\mathrm{PA} \sim 30^{\circ}$ in all of the available emission lines, parallel to the dust lane observed by Veilleux et al. (2001). The emission bends in the southern part towards PA $\sim 210^{\circ}$, especially in [O III], and to $\mathrm{PA} \sim 45^{\circ}$ in the north of the FOV. [O III] has a significant emission extension in the eastern part of the FOV, associated with high receding velocities, while the low-ionisation lines exhibit a blob of emission to the south-west, associated with high extinction.

The maximum of the emission in all lines coincides with the maxima in the electron density, the interstellar reddening, and the velocity dispersion (with the exception of [O III] where the FWHM maximum is shifted by $\sim 1^{\prime \prime}$ to the east, see Fig. 18). At the same time, the maximum emission region is characterised by local minima of diagnostic line ratios (Fig. 17).

The LOS velocity fields (Fig. 18) obtained from different emission lines are similar, characterised by receding motions in the northern part and approaching in the south and east of the FOV. Comparison with the stellar velocities (with their kinematic axis in the NE-SW direction) suggests that the gas has a rotational component aligned approximately with stars. Despite the apparent kinematic similarities, the MR2 spectra had to be considered to correspond to two kinematic systems, by fitting the $[\mathrm{S} \mathrm{II}]$ and $[\mathrm{OI}]$ kinematics independently of $\mathrm{H} \alpha$ and [N II], while the unified fit was impossible. The grouping of the emission lines into the two systems was a compromise between the well-determined positions of lines and the number of parameters needed to place enough constraints on the weak lines and the blended lines. We illustrate the blending of the $\mathrm{H} \alpha$ and [N II] lines in the spectra of Fig. 19. Outside the central 

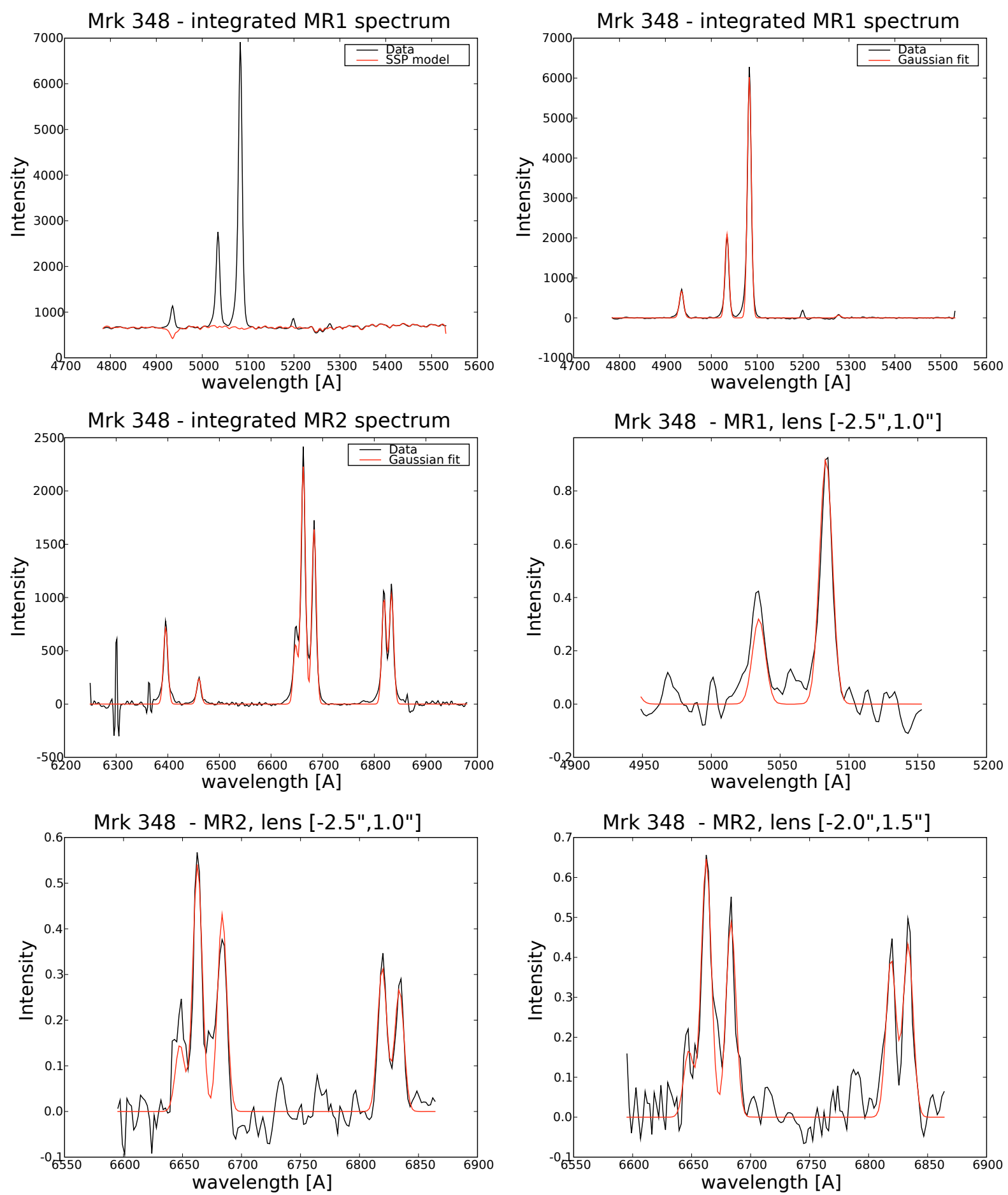

Fig. 12. NGC 262 (Mrk 348).

$\sim 2^{\prime \prime}(350 \mathrm{pc})$, all emission lines are however narrow and nonblended. The differences in the motion of the individual ionic species are depicted in the one-dimensional cut along the major axis (Fig. 18): the velocity curve in [O I] and [S II] is shallower (by $\sim 100 \mathrm{~km} \mathrm{~s}^{-1}$ ) than in the other lines, whereas the most prominent velocities are observed in [O III], especially in the northern part. The velocity curves that we obtained by onedimensional cuts along different axes are consistent with longslit spectroscopy by Márquez et al. (1998).

We detect double-component profiles of emission lines, particularly for $[\mathrm{O} \mathrm{III}]$ and $\mathrm{H} \beta$, while the other lines are too affected by either blending or noise. We show the asymmetric MR1 profiles in Fig. 19, in a combined plot of spectra from three locations. Broad wings of Balmer emission lines were detected in the central $\sim 2^{\prime \prime}(\sim 350 \mathrm{pc})$. However, their relation to BLR emission (already suggested e.g., by Ward et al. 1980; Boisson \& Durret 1986) can only be confirmed after a careful disentangling of all the line components.

Our stellar population modelling revealed spatial variations in the contribution of young stars, which is highest in the strongly emitting region close to the dust lane, while mostly old populations are present in the rest of the FOV (eastern part). 

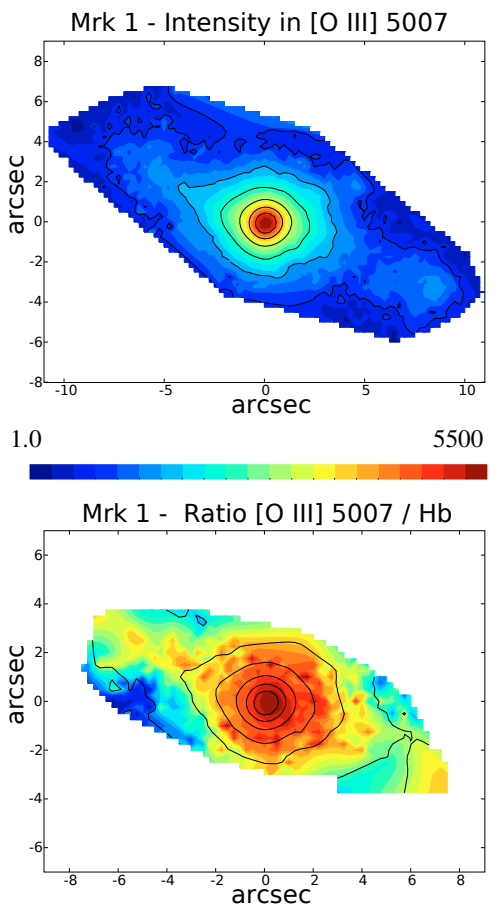

0.6

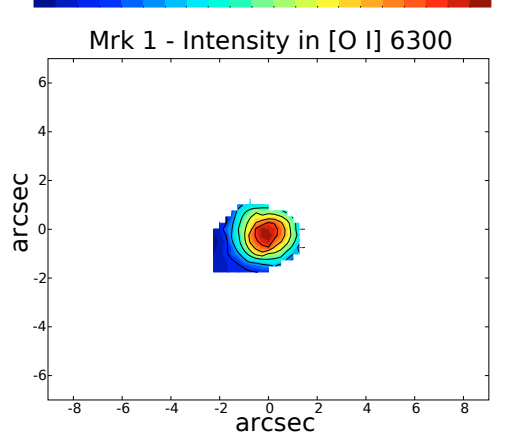

1.1

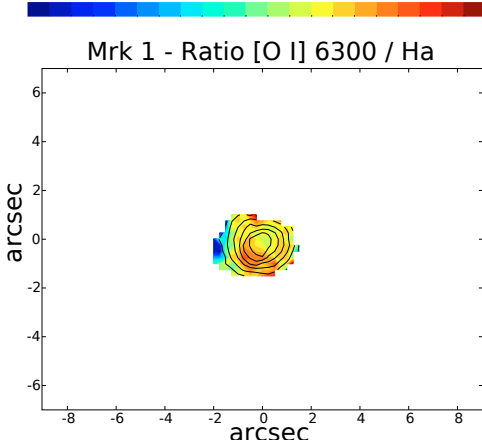

0.1

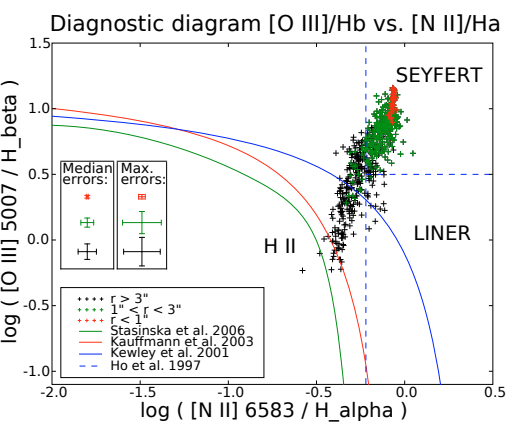

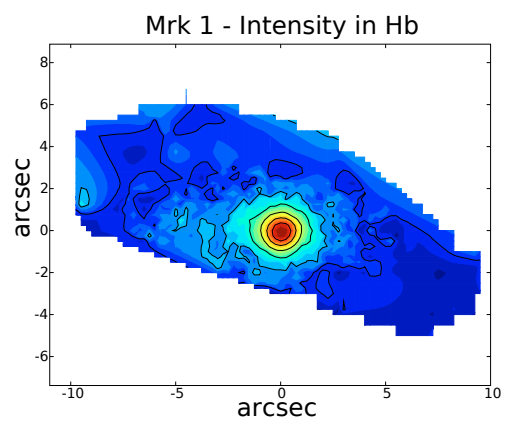

0.9
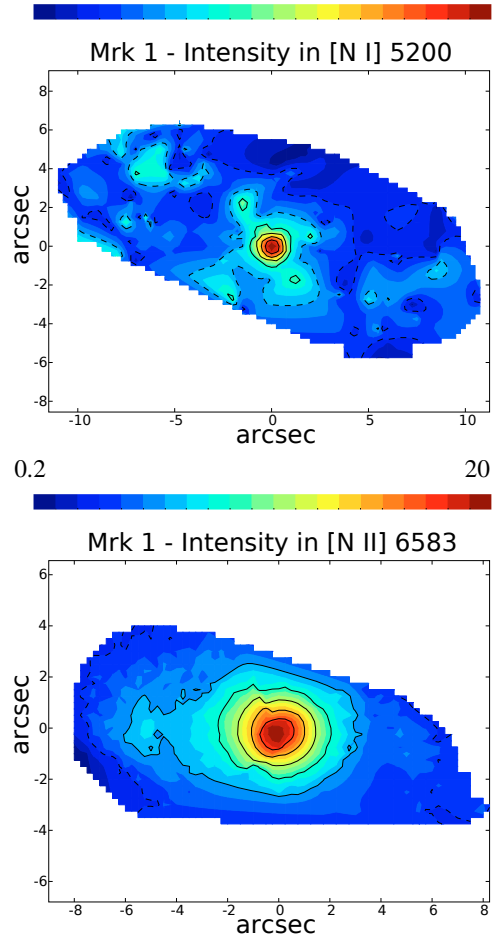

0.2

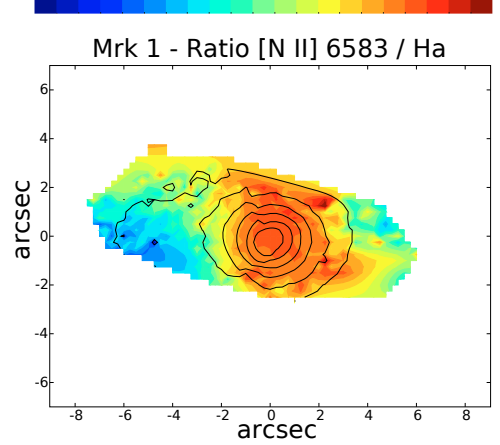

0.3

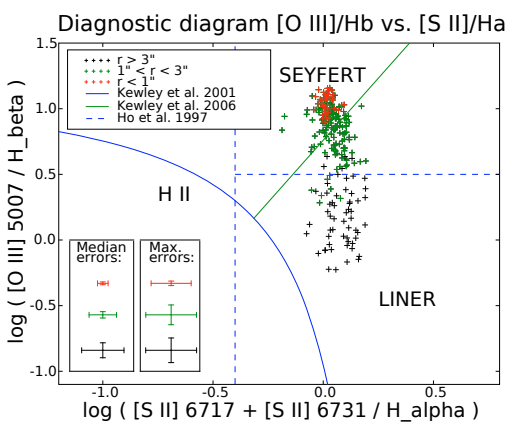

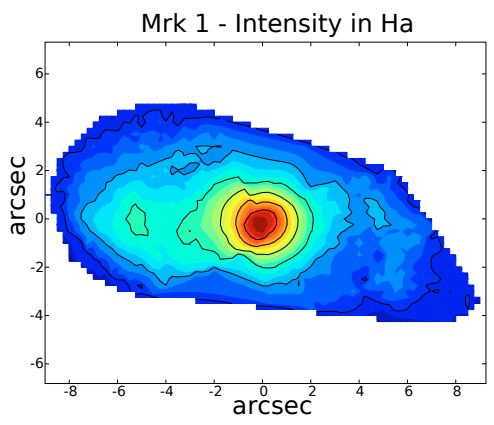

0.5

300

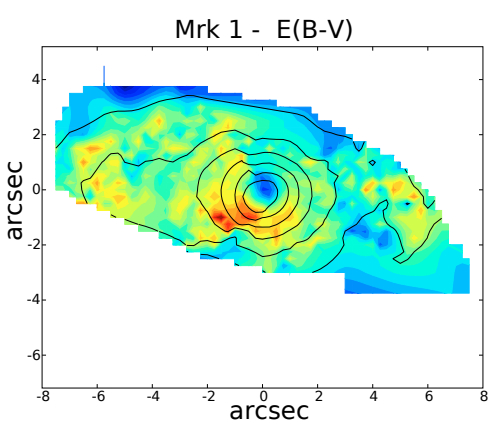

0

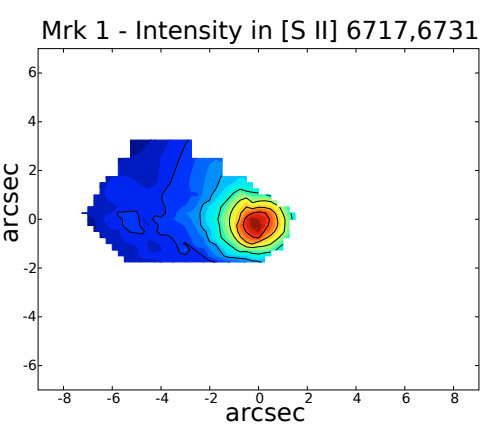

1.1

120

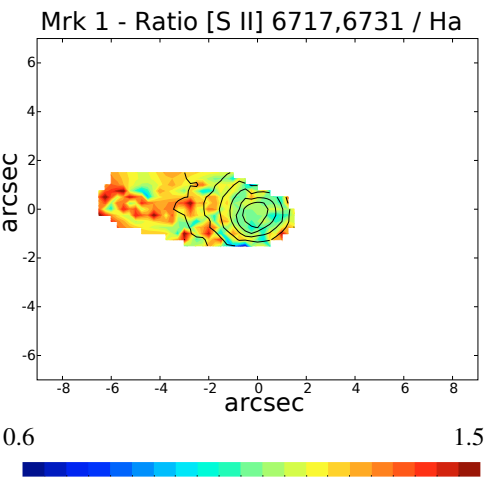

Diagnostic diagram [O III]/Hb vs. [O I]/Ha

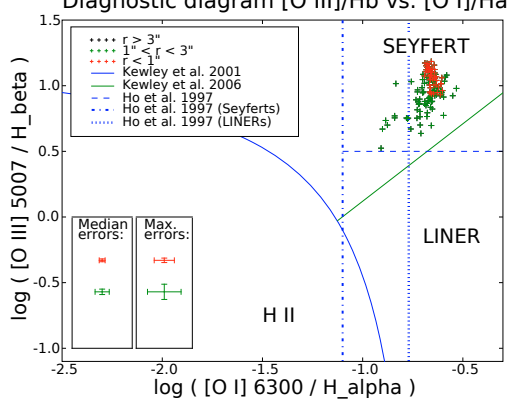

Fig. 13. NGC 449 (Mrk 1). 

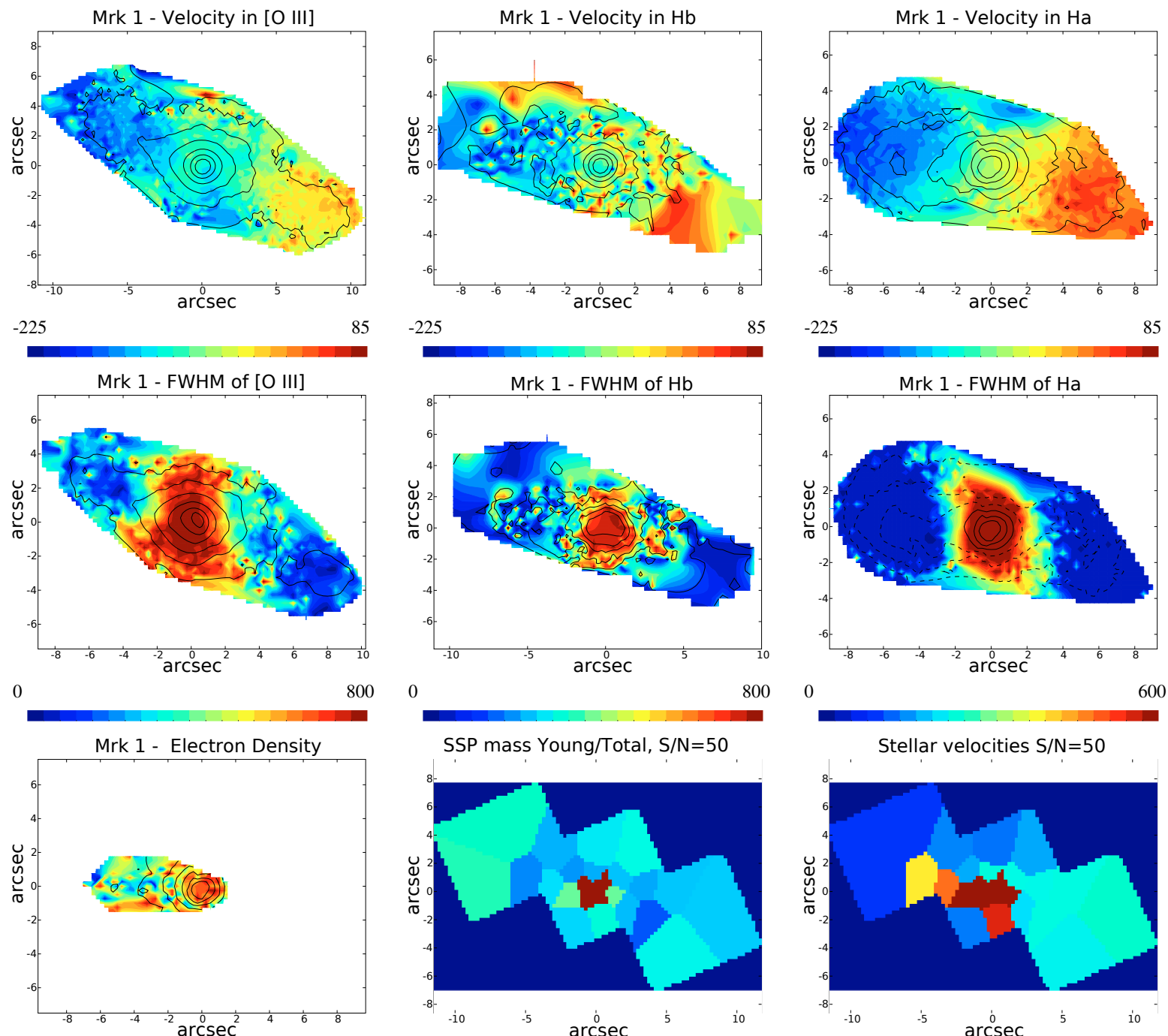

SSP mass Young/Total, $\mathrm{S} / \mathrm{N}=50$

Stellar velocities $\mathrm{S} / \mathrm{N}=50$
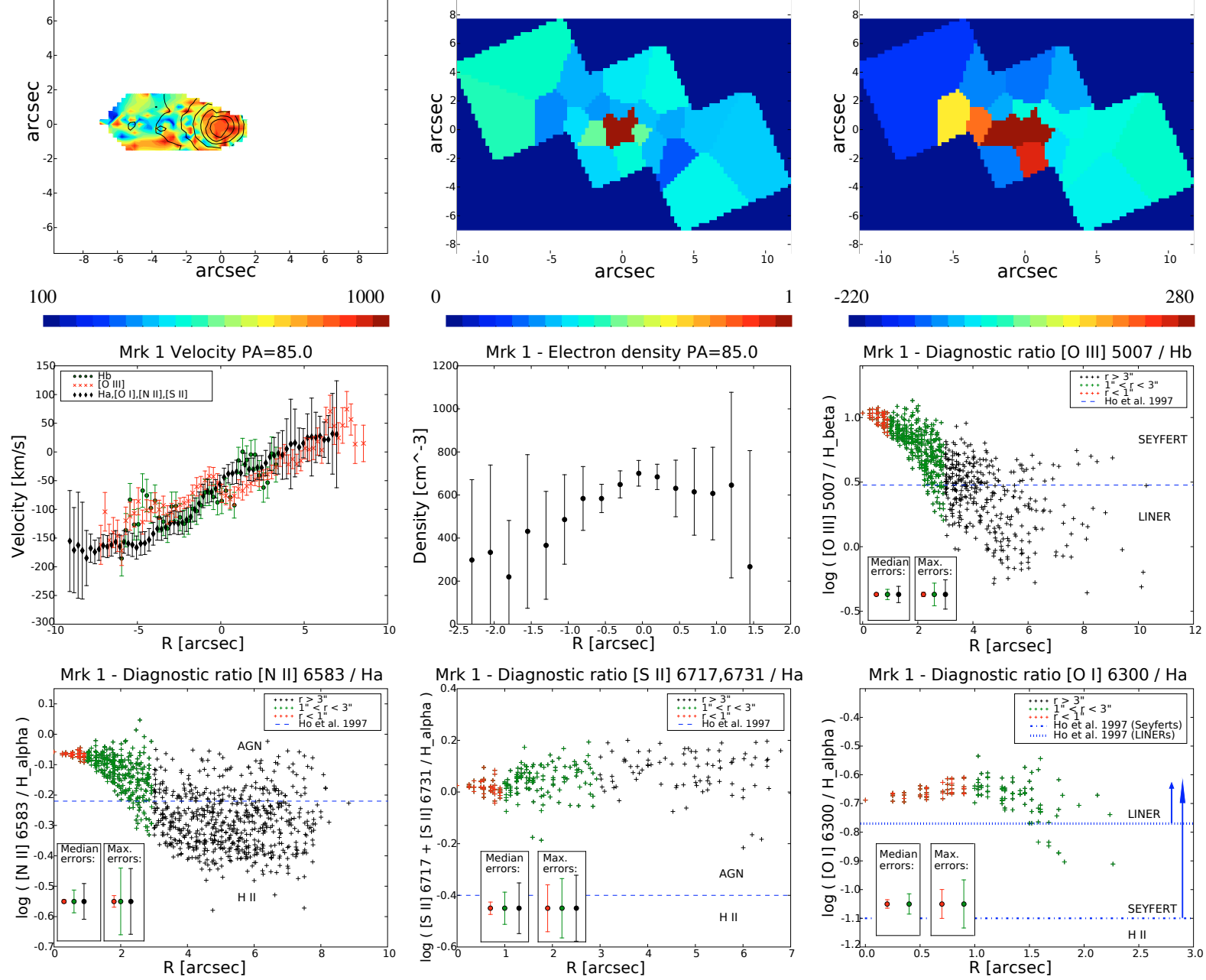

Fig. 14. NGC 449 (Mrk 1). 

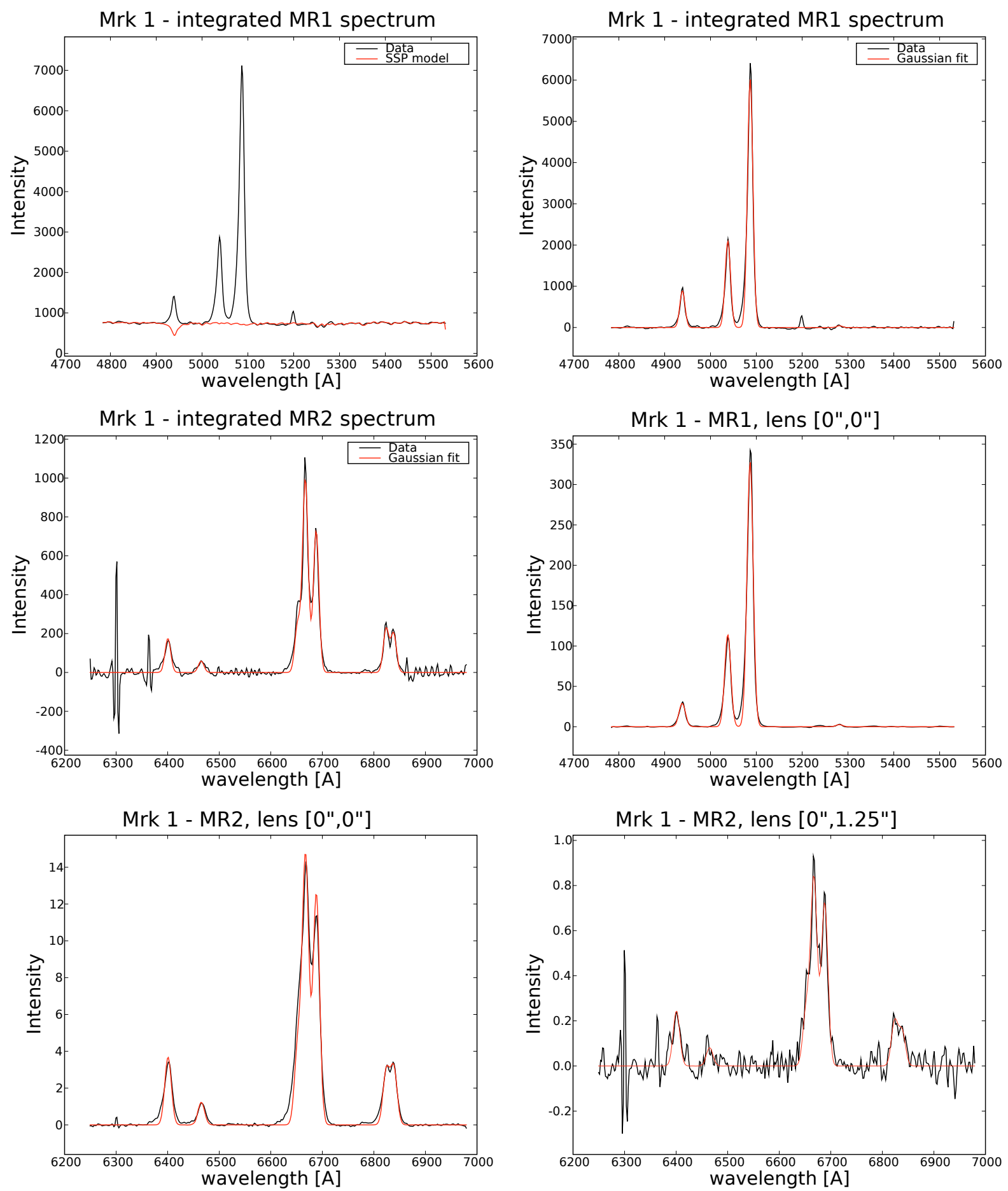

Fig. 15. NGC 449 (Mrk 1).

\section{NGC 3081}

The host galaxy is an early-type spiral with a weak large-scale bar and a nuclear bar (Buta 1990), and four well-defined resonance rings: nuclear, inner and two outer ones (Buta \& Purcell 1998). The brightest emission-line region inside $2^{\prime \prime}$ has a linear morphology, well aligned with the radio emission (Nagar et al. 1999). Slitless spectroscopy by Ruiz et al. (2005) revealed two major kinematic components in the $\left[\mathrm{O}\right.$ III] line at $\sim-250 \mathrm{~km} \mathrm{~s}^{-1}$ and $+50 \mathrm{~km} \mathrm{~s}^{-1}$.

We observed the object in one spectral domain (MR1) only. The photometric axis in both [O III] and $\mathrm{H} \beta$ stretches along the

PA $\sim 155^{\circ}-165^{\circ}$, even though it is less pronounced in [O III] (Fig. 20). Unlike in [O III], the emission in $\mathrm{H} \beta$ is elongated asymmetrically to the south-east.

The mean LOS velocity fields of [O III] and $\mathrm{H} \beta$ (Fig. 20) show similar structures, forming an S-shape in [O III]. However, important differences between [O III] and $\mathrm{H} \beta$ velocities are encountered in one-dimensional cuts of the velocity maps. The $\mathrm{S}$-shape is not reproduced in the stellar velocities, derived from high-quality absorption-line data in this object, which have a rotational pattern with a kinematic axis PA $\sim 90^{\circ}$. The anomalous values of stellar velocities detected in several bins of the map in 


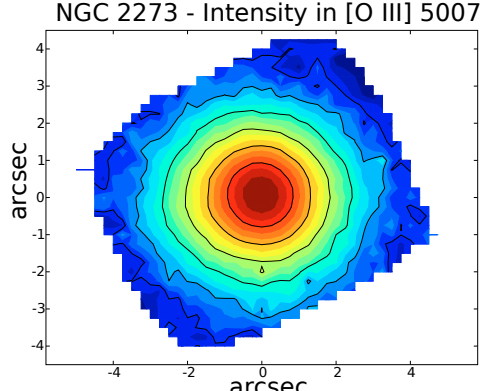

1.5

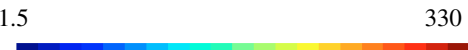

NGC 2273 - Ratio [O III] 5007 / Hb

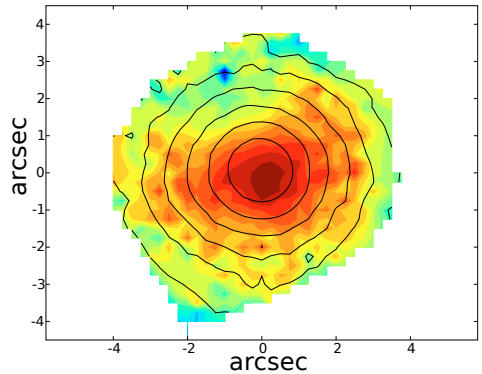

0.1

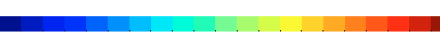

NGC 2273 - Velocity in [O III]

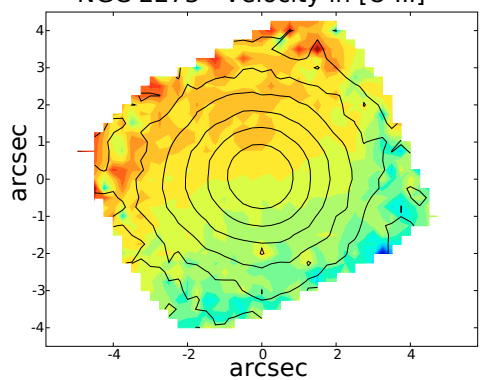

$-205$

275

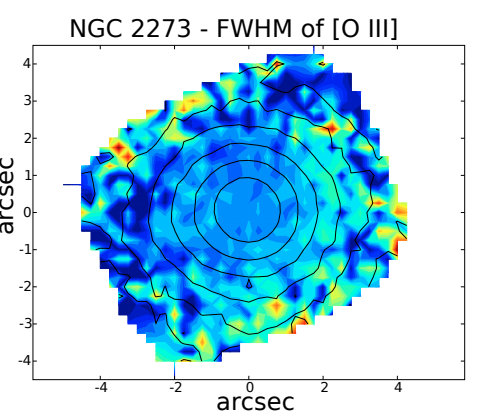

0

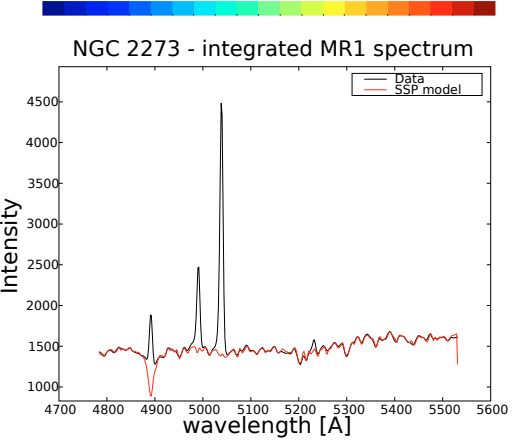

14

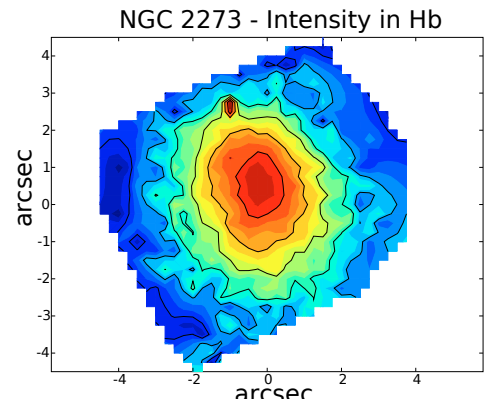

$$
1.5
$$

NGC 2273 - Diagnostic ratio [O III] 5007 / Hb
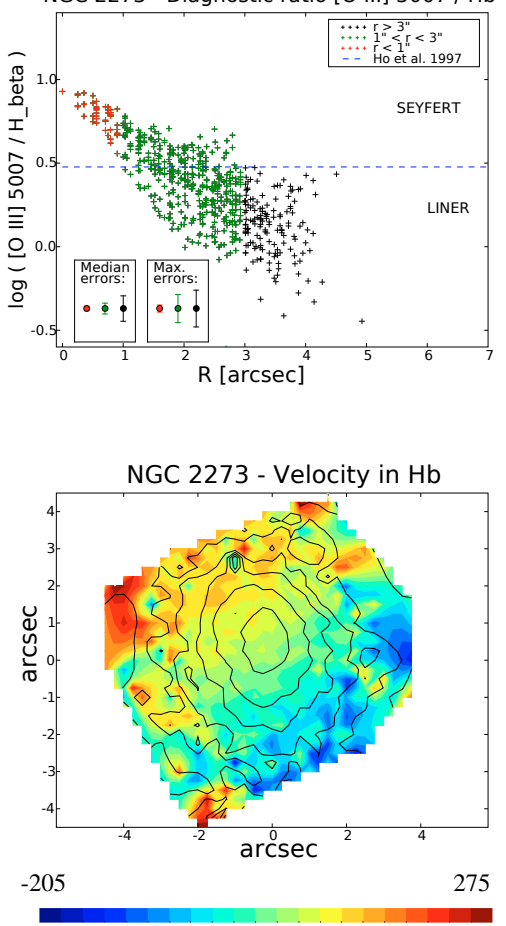

NGC $2273-\mathrm{FWHM}$ of $\mathrm{Hb}$

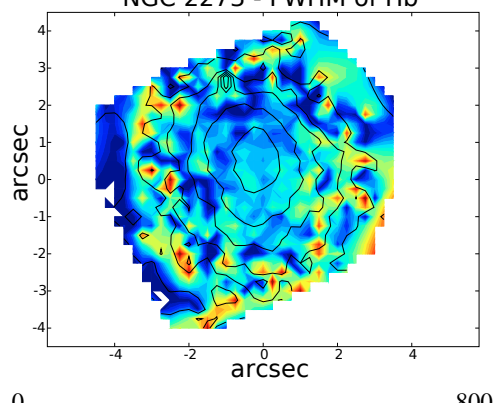

$$
0
$$
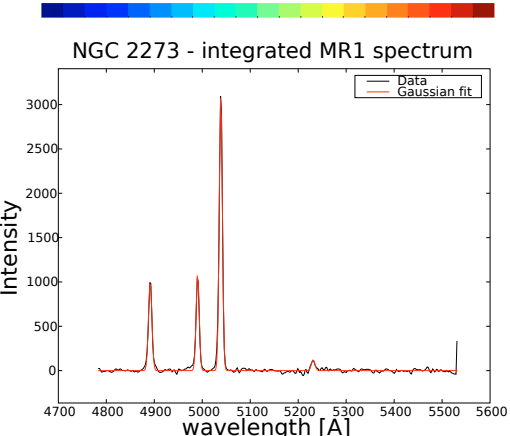
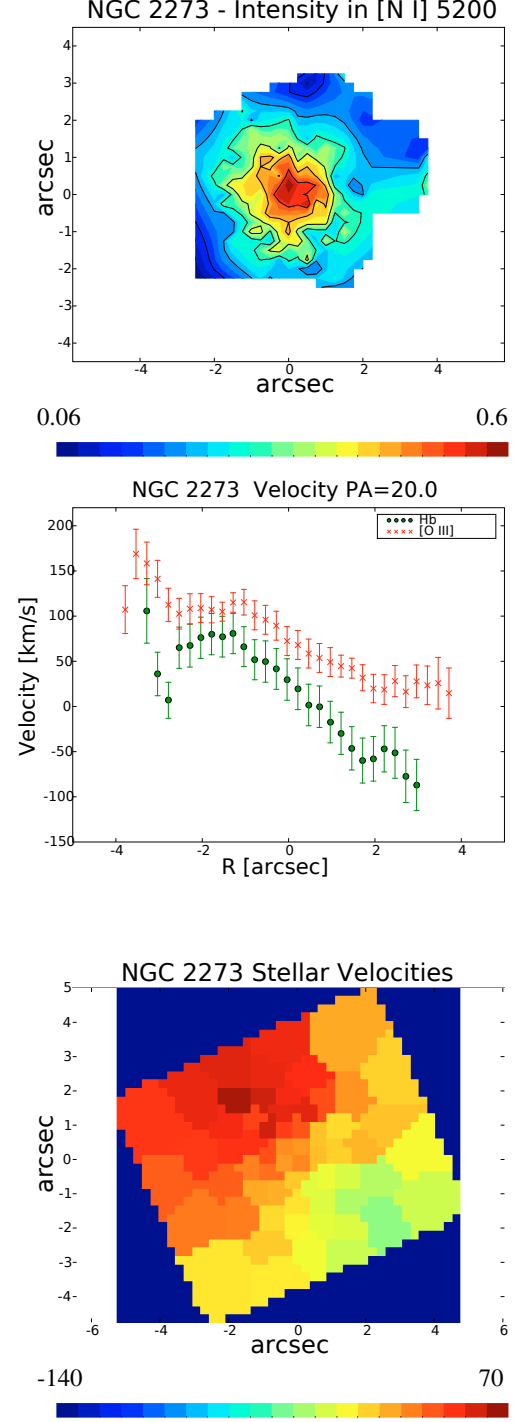

SSP mass Young/Total, $\mathrm{S} / \mathrm{N}=50$

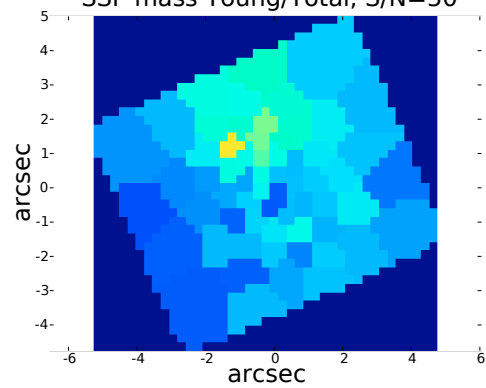

0

NGC 2273 - MR1, lens [0",0"]

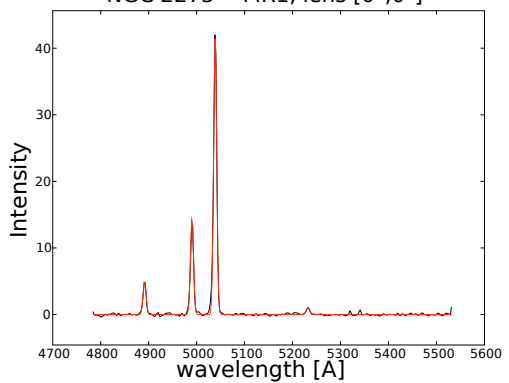

Fig. 16. NGC 2273. 

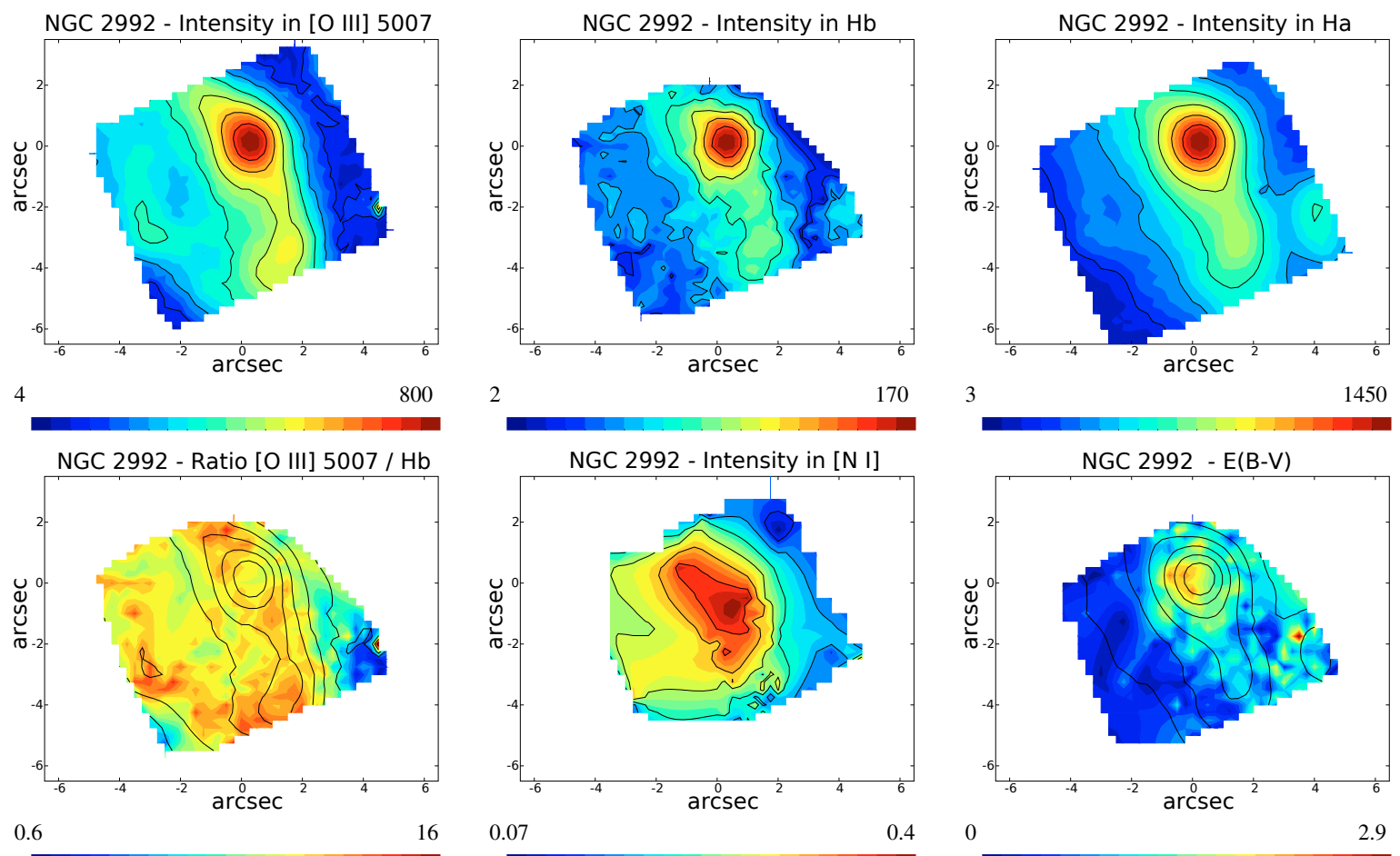

$$
0.07
$$

0.4

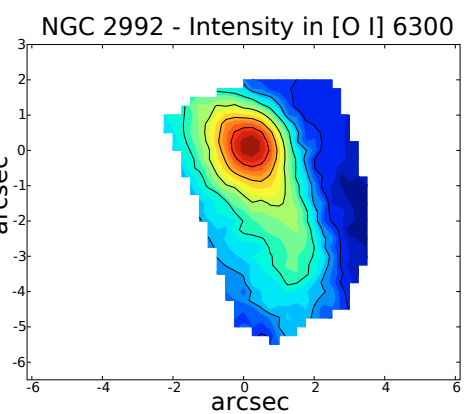

1
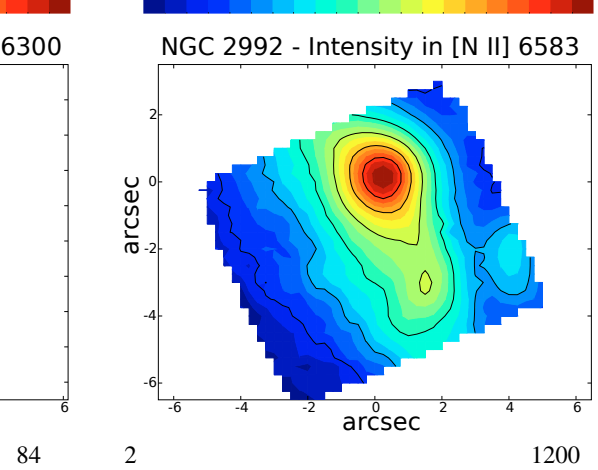

0

2.9

NGC 2992 - Intensity in [S II] 6717,6731

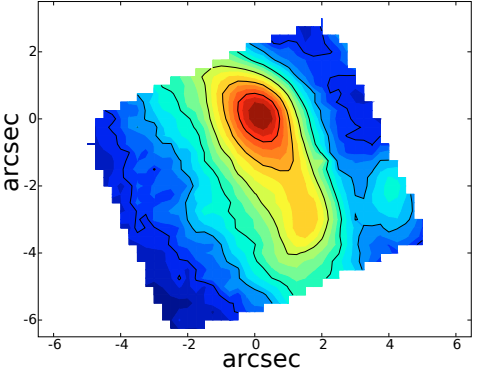

2

1200
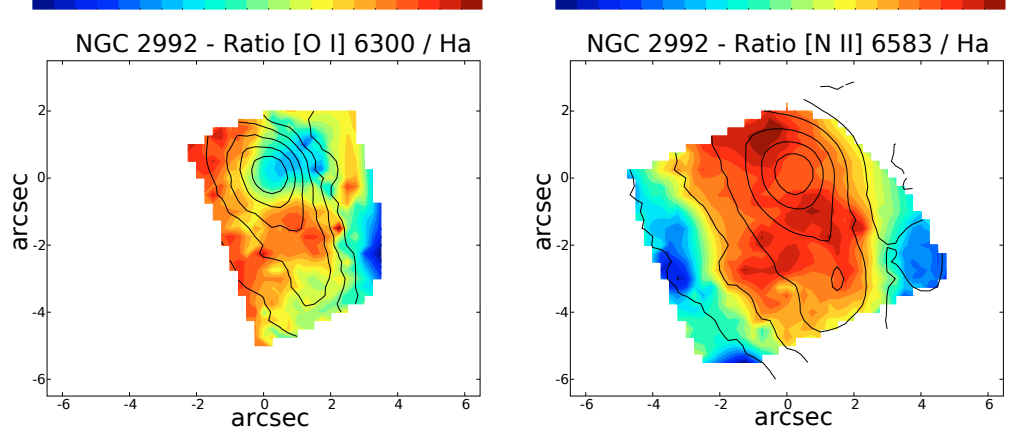

0.3
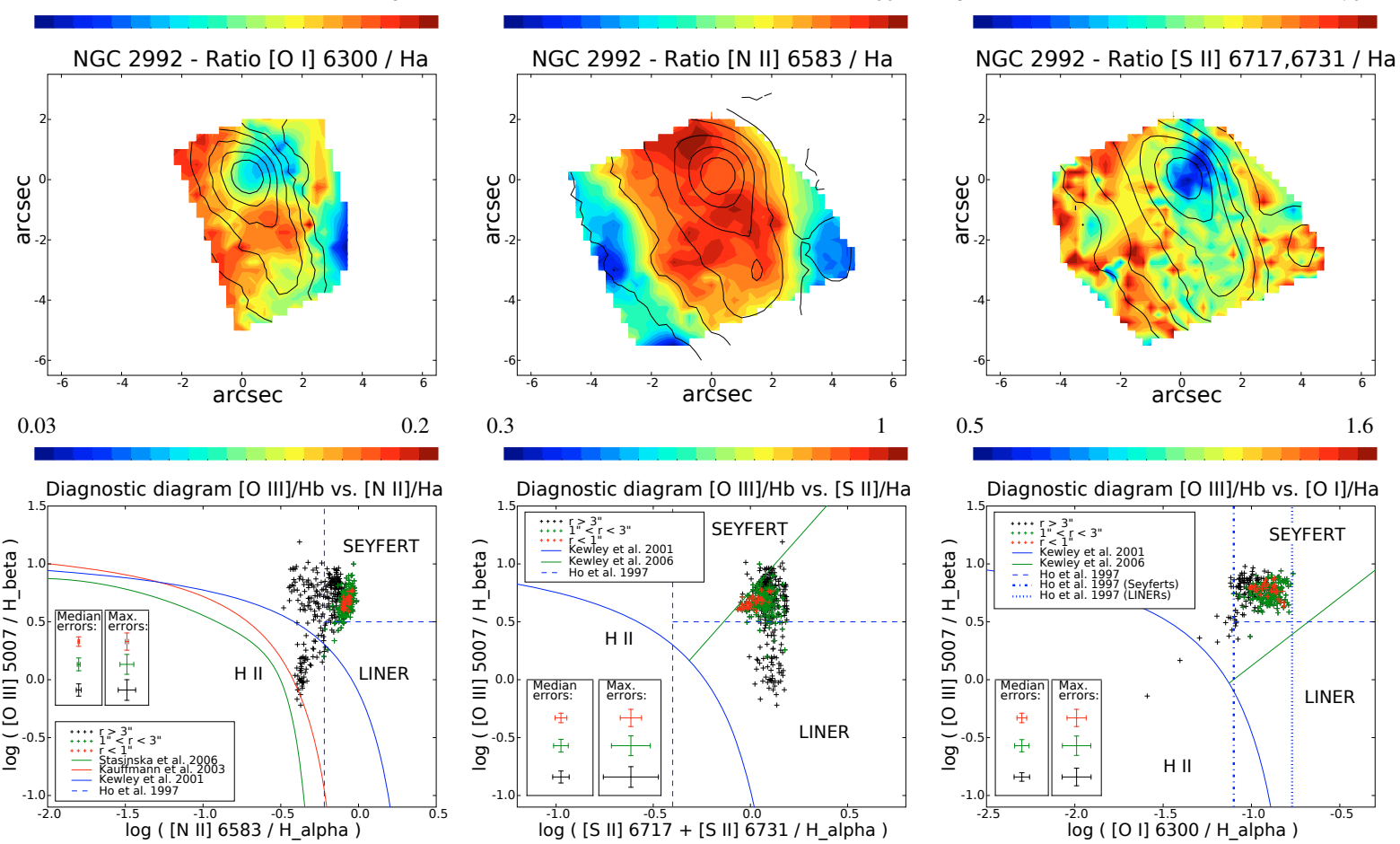

0.5

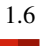

Diagnostic diagram [O III]/Hb vs. [O I]/Ha

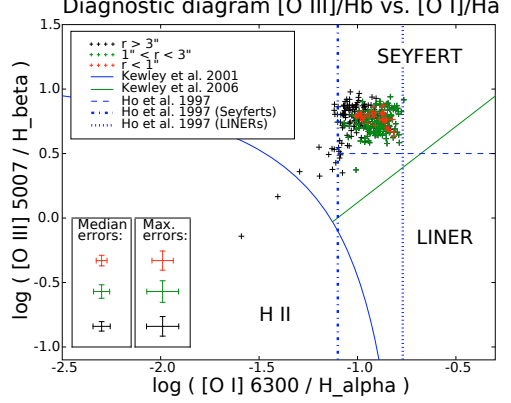

Fig. 17. NGC 2992. 

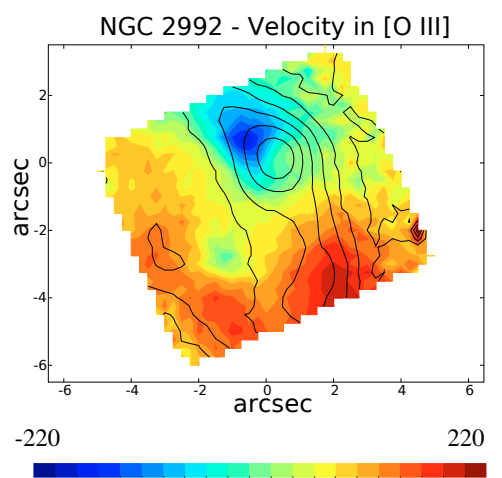

NGC 2992 - FWHM of [O III]
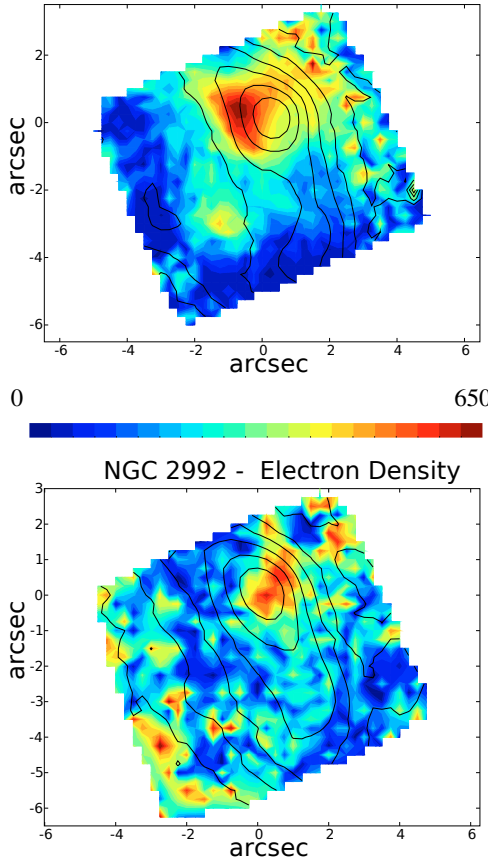

100
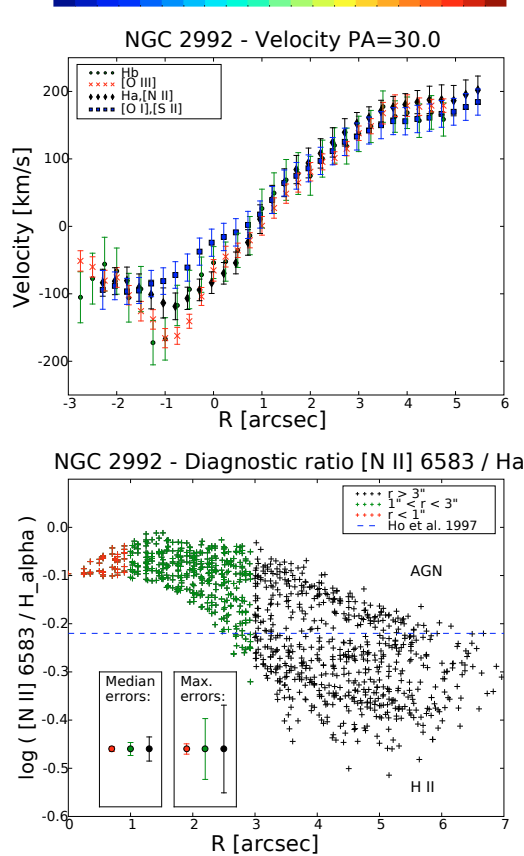
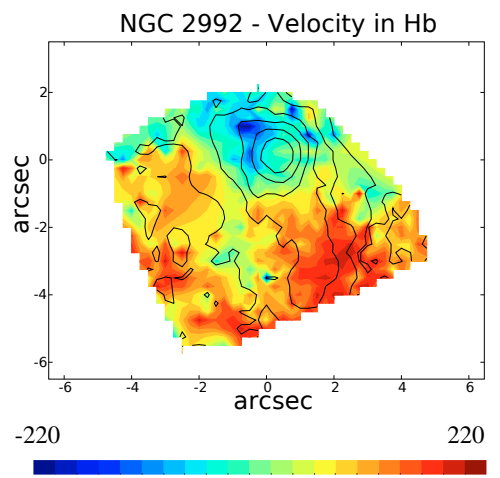

NGC 2992 - FWHM of Hb

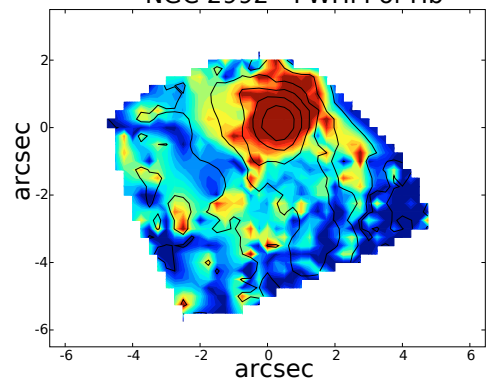

0
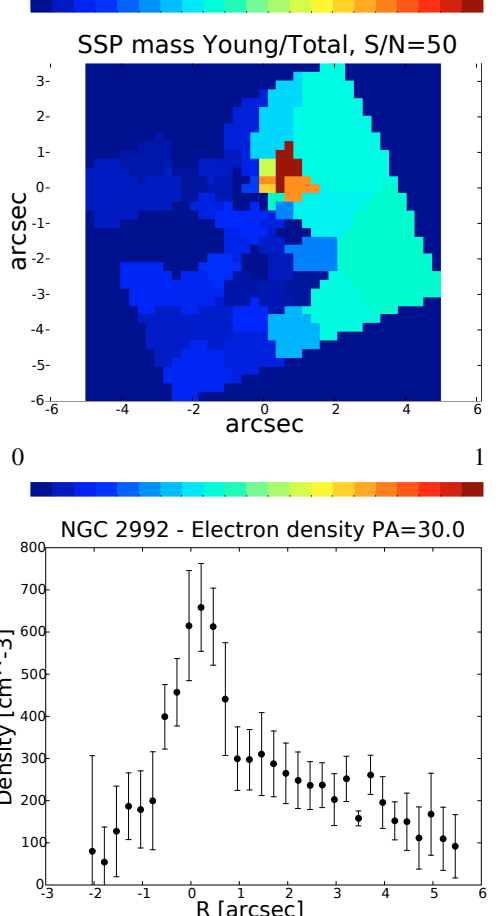

NGC 2992 - Diagnostic ratio [S II] 6717,6731 / Ha

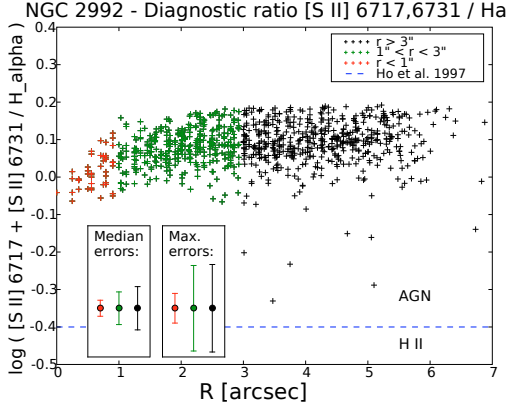

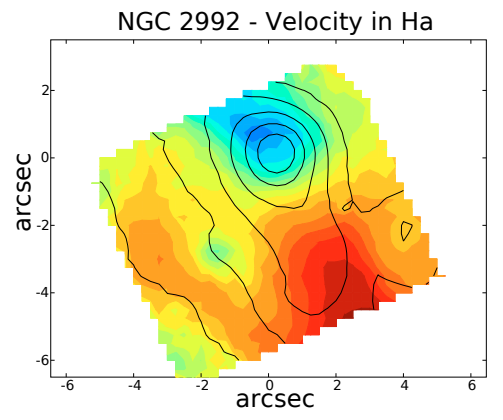
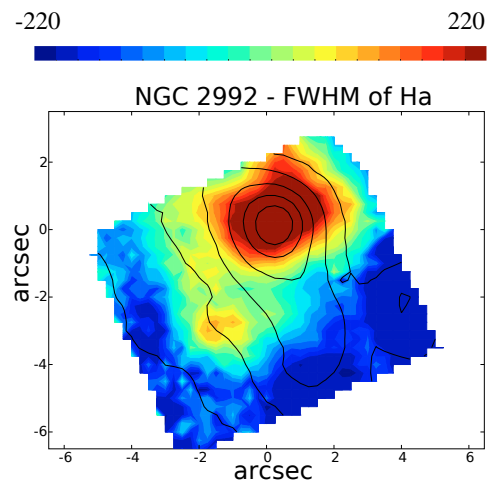

800

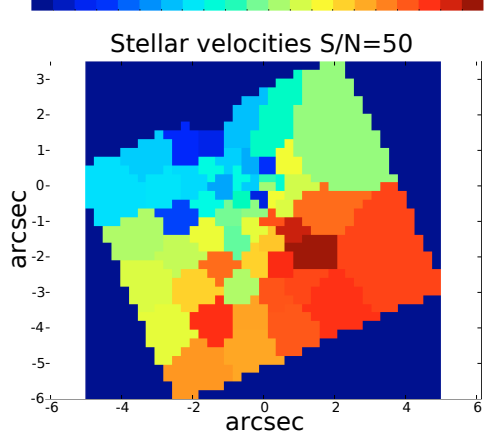

$-110$

\section{0}

NGC 2992 - Diagnostic ratio [O III] 5007 / Hb

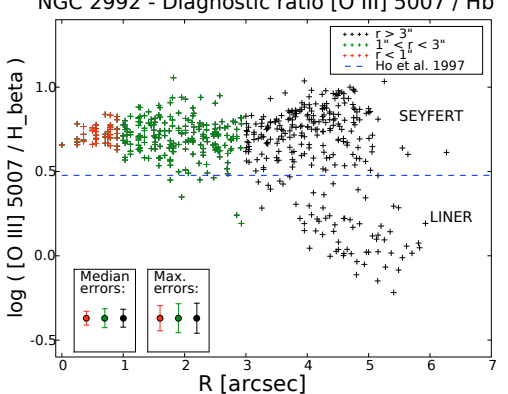

NGC 2992 - Diagnostic ratio [O I] $6300 / \mathrm{Ha}$

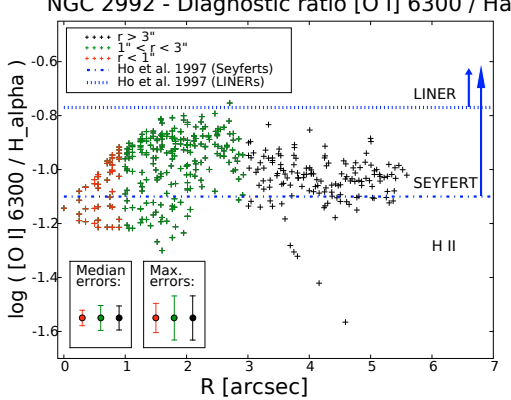

Fig. 18. NGC 2992. 

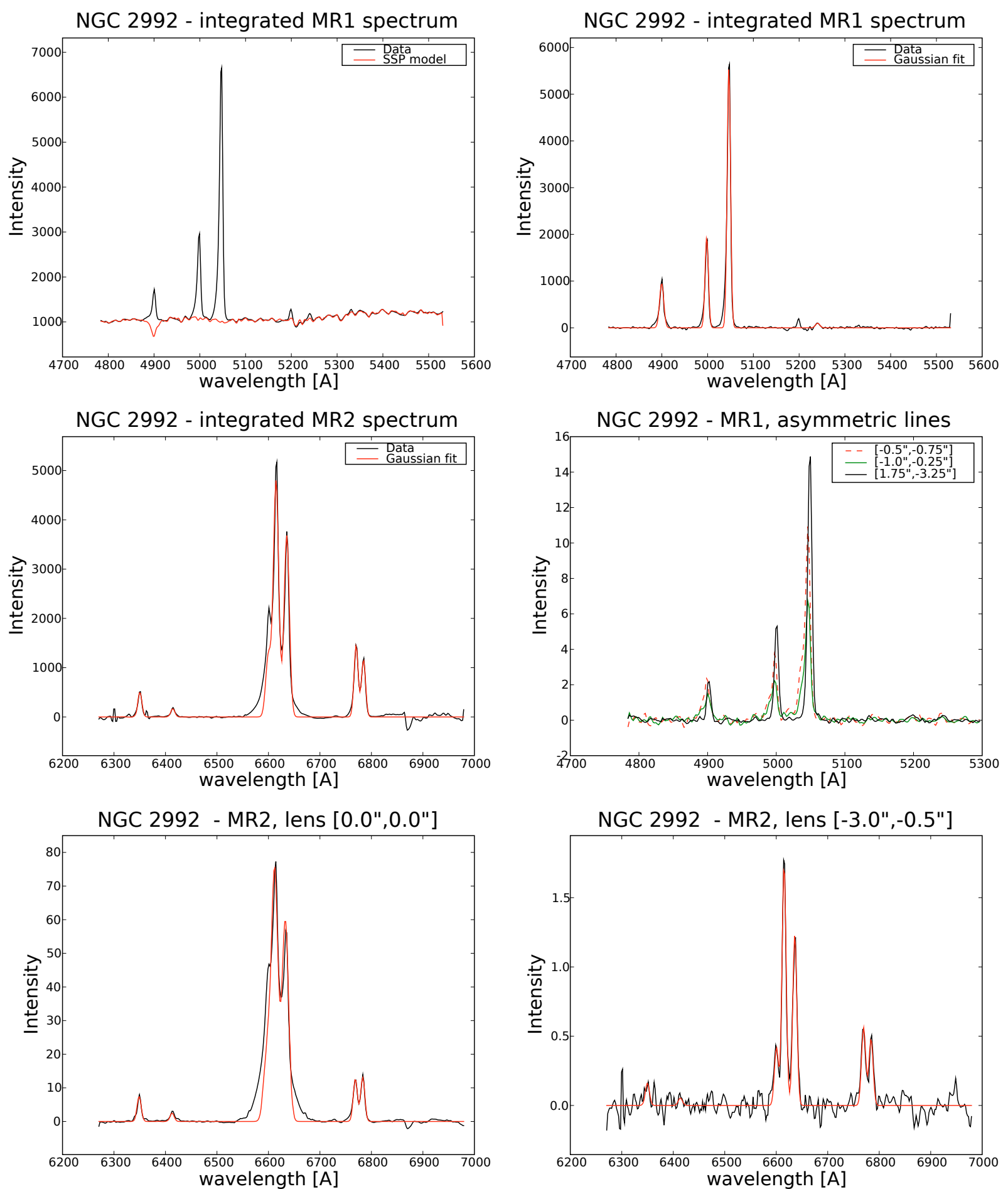

Fig. 19. NGC 2992.

Fig. 20 do not correspond to true velocities, and are results of poor fits in these particular positions. Interestingly, the northern region of receding [O III] velocities is characterised by minimum values of the line width. We found no prominent asymmetries in emission line profiles (Fig. 20).

Our stellar population modelling revealed low contributions from young stars, compared to the other Seyfert nuclei in the studied sample (Fig. 20). Nevertheless, the SSP ages form a clear spatial structure with a boundary at $\sim 800$ pc separating older populations inside this radius and younger outside.

\section{NGC 4388}

The galaxy belongs to the Virgo cluster (Phillips \& Malin 1982; Yasuda et al. 1997). The galactic disc of PA $\sim 90^{\circ}$ is inclined at $i \sim 78^{\circ}$ (Veilleux et al. 1999a), with the north rim on the near side. The nucleus is a strong X-ray source (e.g., Hanson et al. 1990; Takano \& Koyama 1991; Lebrun et al. 1992; Iwasawa et al. 1997). Radio-emission morphology suggests a collimated AGN-driven outflow reaching 200 pc south of the nucleus (e.g., Stone et al. 1988; Falcke et al. 1998). Weak broad H $\alpha$ emission 


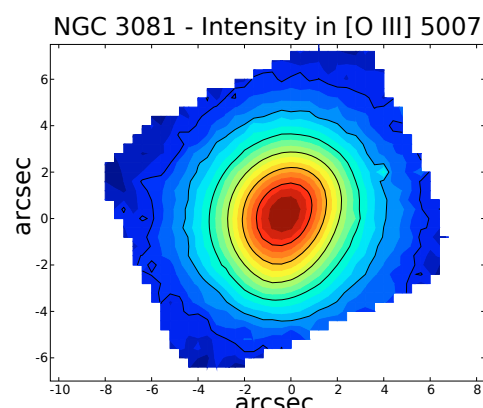

0.9

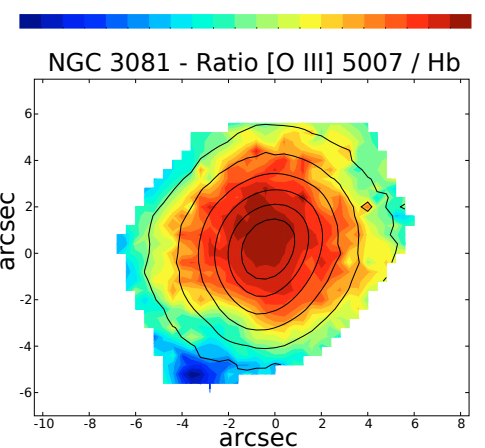

0.3

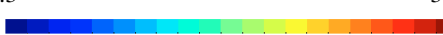

NGC 3081 - Velocity in [O III]

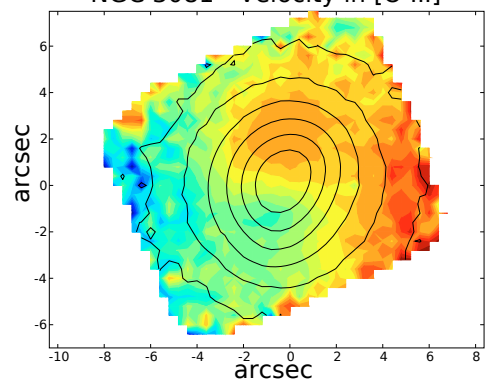

$-170$

130
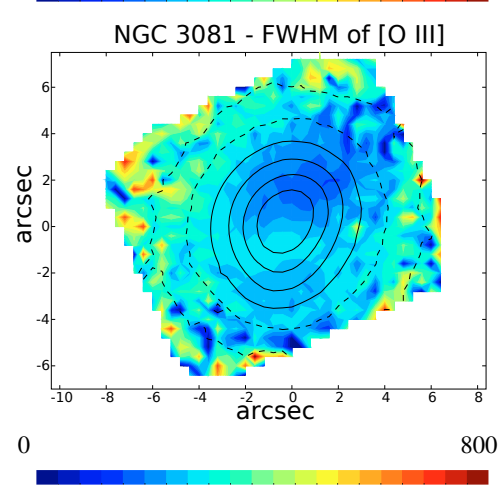

NGC 3081 - integrated MR1 spectrum

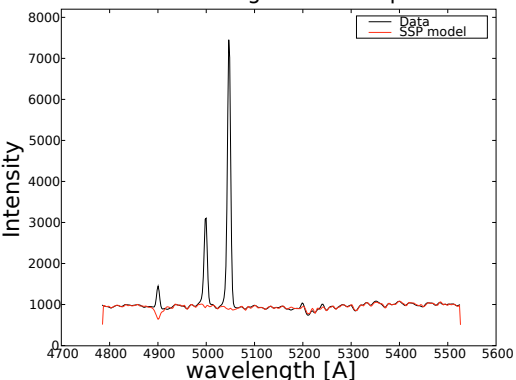

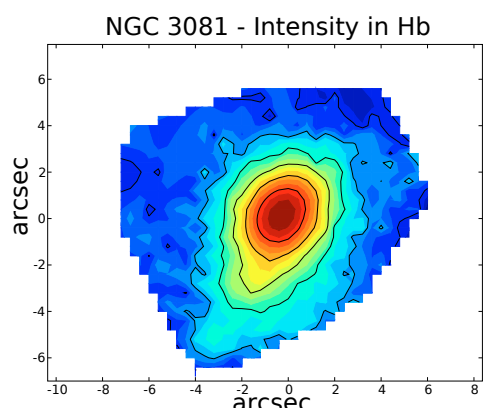

0.6

NGC 3081 - Diagnostic ratio [O III] 5007 / Hb
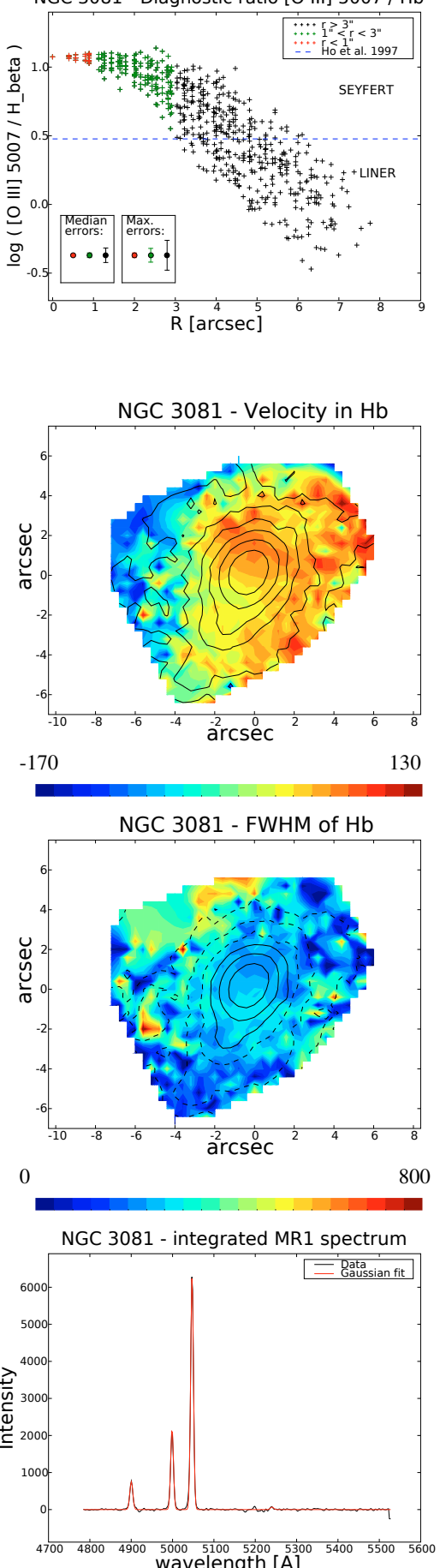
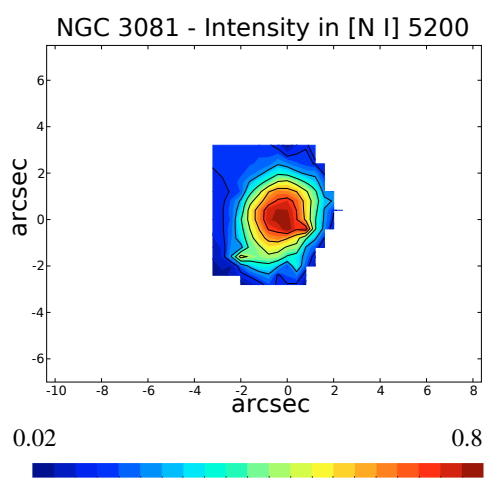

NGC 3081 Velocity PA $=160.0$

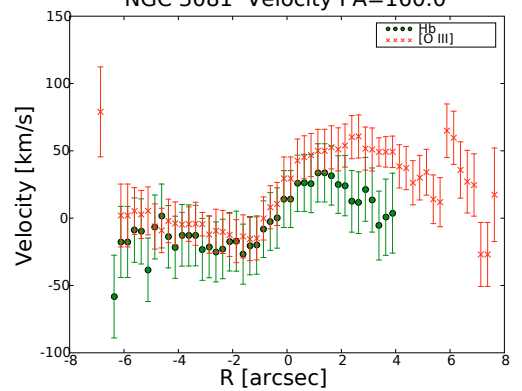

Stellar velocities $\mathrm{S} / \mathrm{N}=50$
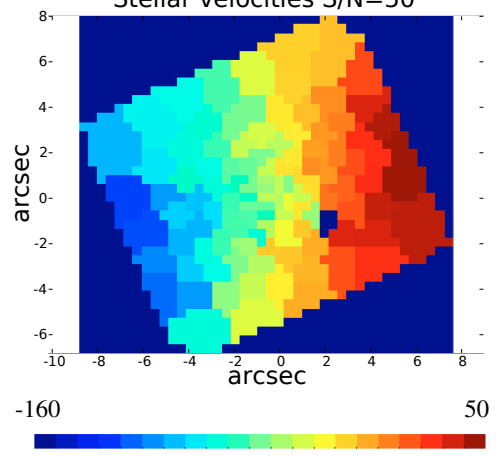

SSP mass Young/Total, $\mathrm{S} / \mathrm{N}=50$
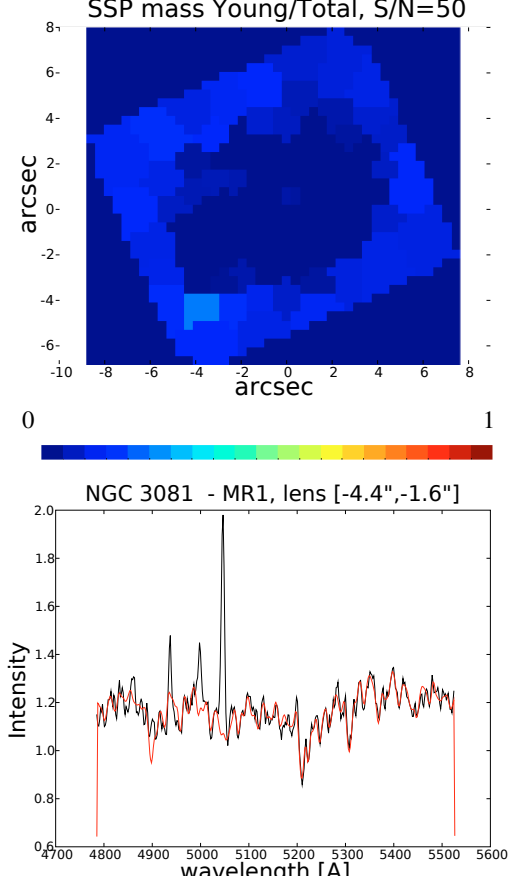

Fig. 20. NGC 3081. 
implies that a hidden type-1 nucleus is present (Filippenko \& Sargent 1985). Extended optical emission has two components of different excitation, one associated with the galactic disc and another reaching 50" (4 kpc) above the galactic plane in the form of two opposite cones (e.g., Pogge 1988; Corbin et al. 1988; Falcke et al. 1998; Veilleux et al. 1999b). While Rubin et al. (1997) argued that anomalous kinematics in the central parts of the galactic disc were indicative of presence of a discrete, rapidly rotating circumnuclear disc, Veilleux et al. (1999a,b) found that the gas kinematics were consistent with elliptical streaming in the bar potential, plus a bipolar outflow extending out of the galactic plane (and excluded other models for the extragalactic component). They argued that the bar-induced non-circular motions may account for the emission-line splitting of the maximum amplitude of $\sim 150 \mathrm{~km} \mathrm{~s}^{-1}$ detected symmetrically out to radii of $\sim 10^{\prime \prime}(\sim 1 \mathrm{kpc})$ along the disc, i.e., close to the end of the bar.

In the case of this nearby galaxy, our FOV is restrained and covers the central $\sim 400$ pc only, which is insufficient for interpreting the large-scale structures known from e.g., Veilleux et al. (1999b), but we sample the central region with the spatial scale of $\sim 80 \mathrm{pc}$, obtaining thus a highly detailed picture. The maximum of emission within our FOV is located in the south-west (Fig. 21), and the emission intensity isocontours have strongly irregular forms, pointing to the north-east PA $\sim 30^{\circ}-45^{\circ}$, i.e., a direction consistent with the extragalactic component reported by Veilleux et al. (1999b). The $\mathrm{H} \beta$ morphology differs slightly from [O III] in the norther part of the FOV, where it bends by $\sim 90^{\circ}$ at $\sim 200 \mathrm{pc}$. The map of $[\mathrm{O} \mathrm{III}] / \mathrm{H} \beta$ ratio reveals high ionisation in the region of maximum [O III] emission extending along PA $\sim 45^{\circ}$ (Fig. 21).

Due to good-quality stellar data in this object, our map of mean LOS stellar velocities exhibits a clear pattern, which is similar to that observed in $\mathrm{H} \alpha$ emission by Veilleux et al. (1999b). Because of the large FOV covered by the Fabry-Perot interferometer observations, they were able to identify a strongly $\mathrm{S}$-shaped form in the isovelocity contours in the galactic disc. For the gas velocities that we observe, our limited FOV only allows us to identify agreement between the general orientations of the central velocity features in our data and those of Veilleux et al. (1999b).

We detect two regions of high velocity dispersion, especially in [O III] maps: in the south close to the peak emission, and in the north of the FOV. These regions are characterised by anomalous emission-line profiles, the former with weak red wings, the latter with a marked additional blueshifted component separated by $\sim 800-900 \mathrm{~km} \mathrm{~s}^{-1}$ from the main emission component both in [O III] and $\mathrm{H} \beta$ (which is significantly higher than reported by Veilleux et al. 1999b). Examples of spectra and their one-component fits are presented in Fig. 21, from which it is clear that the velocity maps follow the strongest component of the lines. The additional redshifted and blueshifted components are restricted to the two regions and never outshine the main component.

The stellar population modelling shows the largest contribution of young stars ( $\sim 10 \%$ of mass) close to the point of inversion of the stellar velocities. The observed field is otherwise dominated by old stars, consistent with the findings of Storchi-Bergmann et al. (1990).

\section{NGC 5728}

The host galaxy has a bar along $\mathrm{PA}=33^{\circ}$, a nuclear bar at PA $86^{\circ}-90^{\circ}$ (Wozniak et al. 1995; Emsellem et al. 2001), and three rings. The nuclear ring has dimensions $9^{\prime \prime} \times 7^{\prime \prime}$, and the major axis at PA $=20^{\circ}$. The Seyfert core is known as one of the most spectacular examples of the biconical emission-line structure in optical observations, with the north-west cone at $\mathrm{PA}=304^{\circ}$ and the south-east cone at $\mathrm{PA}=118^{\circ}$, and opening angles $\sim 55^{\circ}-65^{\circ}$ (Pogge 1989; Wilson et al. 1993). The emission maxima in both $\mathrm{H} \alpha+\left[\mathrm{N}\right.$ II] and [O III] are located $\sim 1^{\prime \prime}$ from the cones' apex (Wilson et al. 1993).

The kinematics of NGC 5728 has been characterised by controversial conclusions, and so far none of the proposed models (radial outflow, inflow or non-axisymmetric rotation in a barred potential) has managed to account fully for all the observational results. The inflow model is supported by the observed asymmetry in the line-emission distribution and the degree of ionisation (Schommer et al. 1988; Wilson et al. 1993; Riffel et al. 2008): the shorter and less ionised north-west cone can be explained by obscuration and interaction with the galactic disc if the north-west is the far side (see Figs. 22 and 23 for a better understanding). On the other hand, HST observations revealed a sharp apex to the north-west cone, whereas the south-east cone terminates with a blunt end $0.5^{\prime \prime}$ from the core, suggesting obscuration by foreground material (Wilson et al. 1993). Together with an excellent alignment with the radio emission, this might be considered evidence of an outflow (Schommer et al. 1988).

Observed with the use of the Fabry-Perot spectrometer by Schommer et al. (1988), strongly S-shaped $\mathrm{H} \alpha$ isovelocity contours in the central $10^{\prime \prime}(2 \mathrm{kpc})$ suggested that these features originated in the bar potential. On the other hand, the bar is in a position for which models by Roberts et al. (1979) do not predict velocity field distortions, therefore Schommer et al. (1988) drew no conclusions about the importance of the bar. SPH simulations by Pérez et al. (2004), which included a bar, failed to reproduce the observed velocity field and the authors proposed that either an additional nuclear bar or radial flows are present.

The major axes of the emission structures determined from our OASIS data are aligned at PA $\sim 125^{\circ}$ (as measured from the outer contours). The emission is asymmetric in the two lobes, as reported before in the literature (see above). The northwest lobe shows an elongation at PA $\sim-40^{\circ}$ in the inner $4^{\prime \prime}$ ( $\sim 800 \mathrm{pc})$, consistent with the HST observations of Wilson et al. (1993), while the more prominent south-west lobe bends towards PA $\sim 115^{\circ}$. We partially resolved the secondary maximum of emission located $\sim 2^{\prime \prime}$ north-west of the maximum, which was reported e.g., by Wilson et al. (1993). The hydrogen emission maps show a prominent arc perpendicular to the lobes, at a distance of $\sim 5^{\prime \prime}(1 \mathrm{kpc})$ north-west of the centre. We interpret this as part of the ring reported e.g., by Wilson et al. (1993).

The gas velocity maps and their one-dimensional cuts (Fig. 23) show a strong gradient of LOS velocities along the major axis of emission, in the vicinity of the dynamic centre of the galaxy. The velocity maxima of opposite signs (approximately $\pm 300 \mathrm{~km} \mathrm{~s}^{-1}$ ) are separated by only $400 \mathrm{pc}$ and are located at the primary and secondary maxima of emission intensity. A comparison with the Fabry-Perot observations (Schommer et al. 1988) provides a different view of the velocity maps across a wider field, providing thus a larger context which is inaccessible with our limited FOV: a strong S-shape is found, which must be taken into account in the interpretation (in a future paper). The hydrogen velocity field of the outer parts of our FOV (outside the central $500 \mathrm{pc}$ ) has an orientation corresponding to the stellar motions that we measured. We suppose that the interpretation of the kinematic structure of NGC 5728 will be aided by rigorous disentangling of multiple-component emission lines that we found to be distributed within an elongated region approximately 

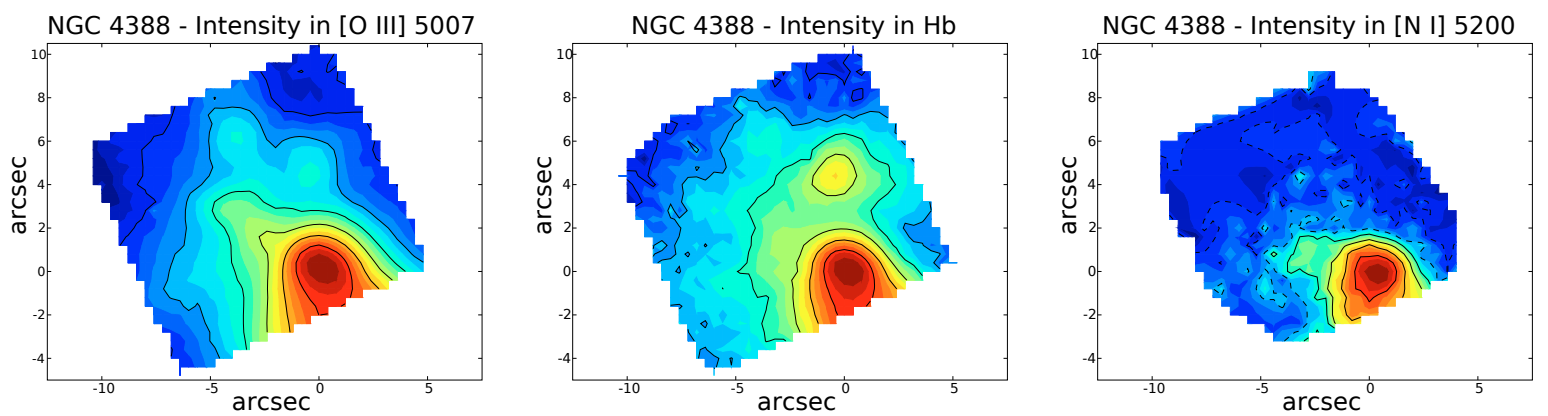

2

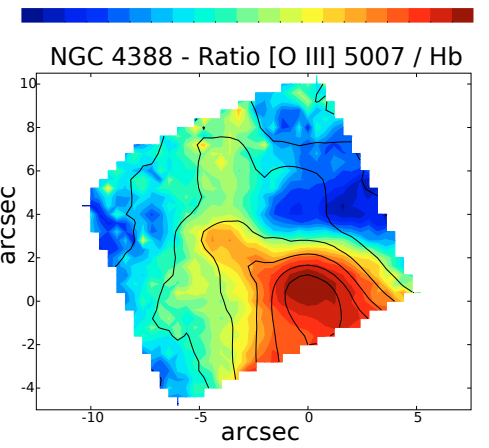

0.9

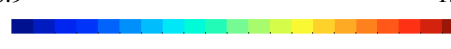

NGC 4388 - Velocity in [O III]

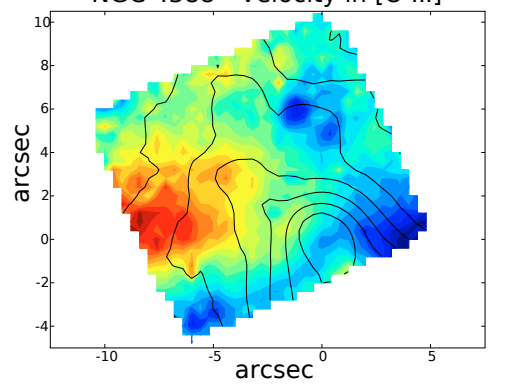

$-70$
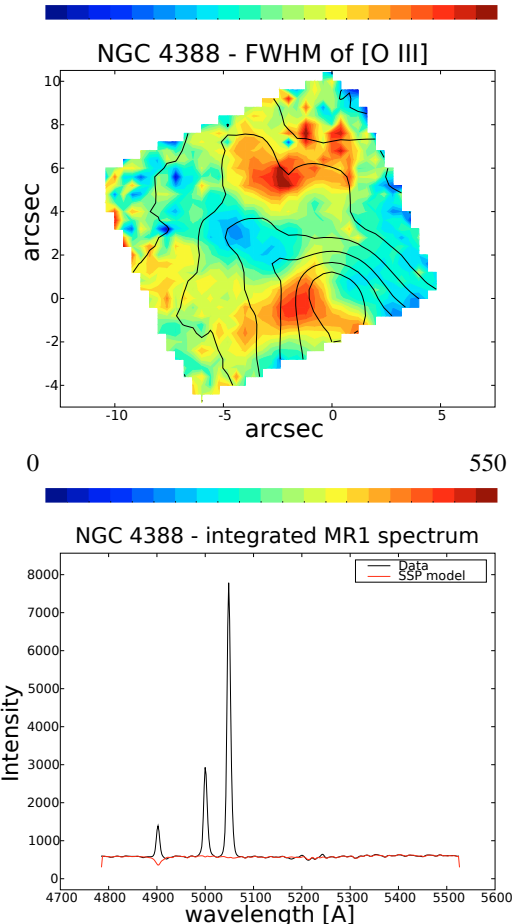

$5000 \quad 5100 \quad 5200 \quad 5300$
wavelength $[A]$

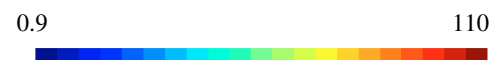

NGC 4388 - Diagnostic ratio [O III] 5007 / Hb
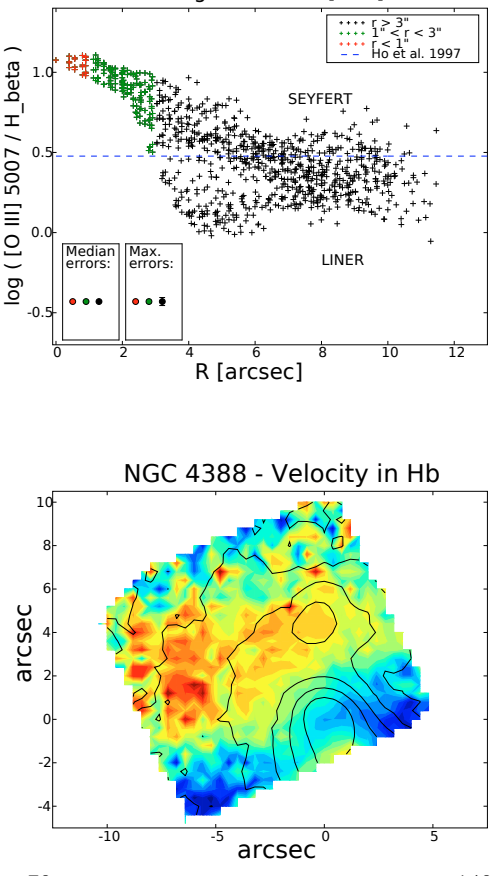

$-70$
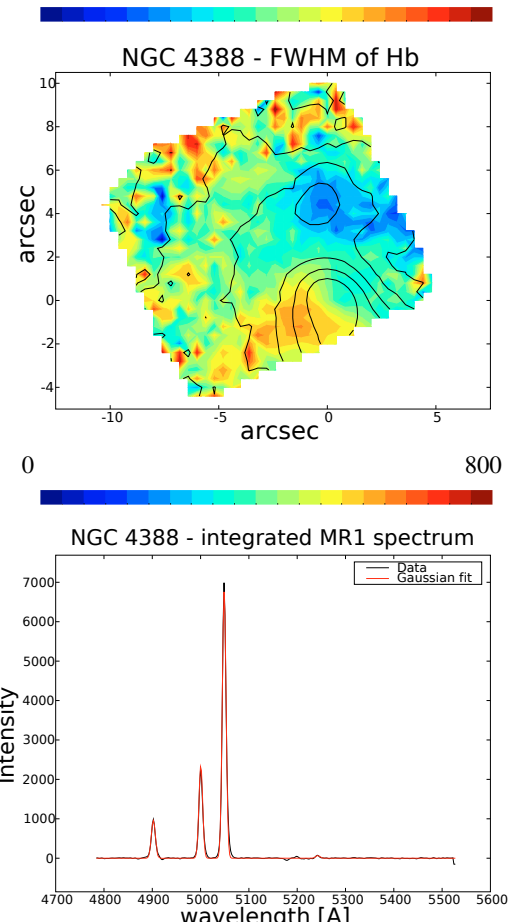
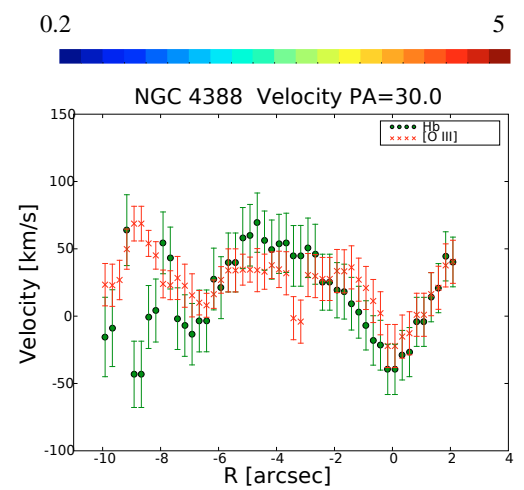

Stellar velocities $\mathrm{S} / \mathrm{N}=50$

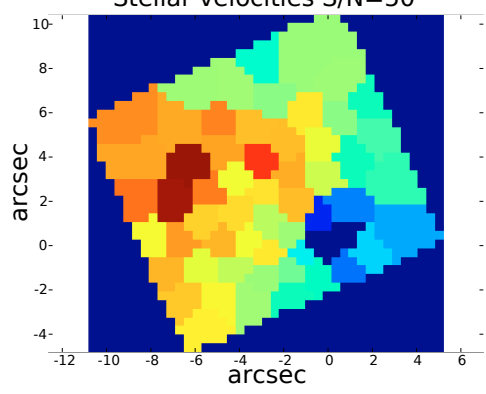

$-100$
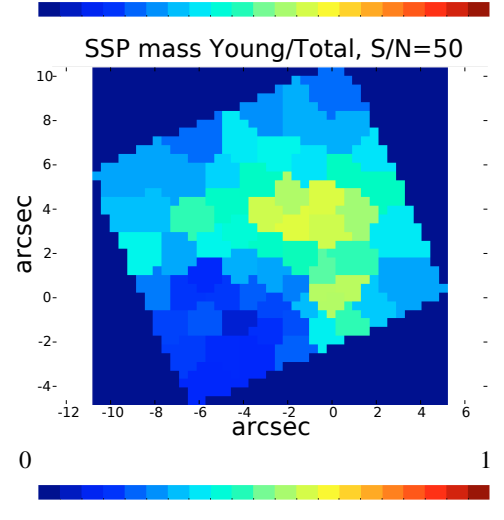

NGC 4388 - MR1, lens [0.4",6.8"]

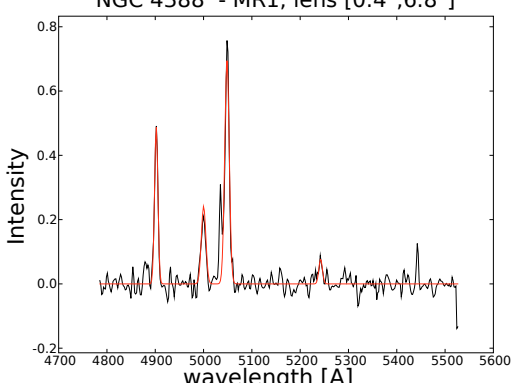

Fig. 21. NGC 4388. 


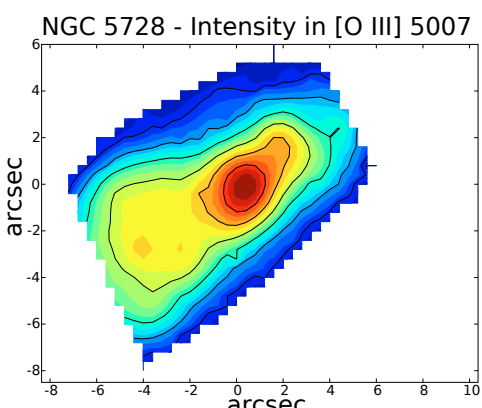

2

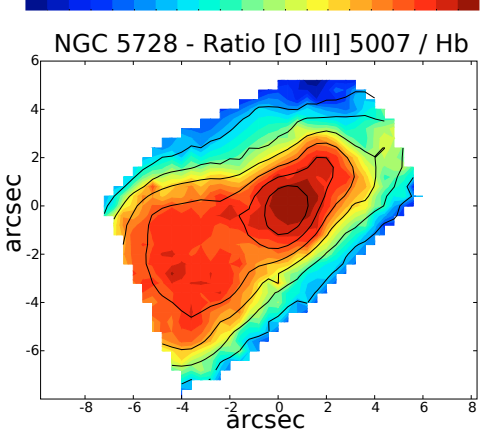

0.3

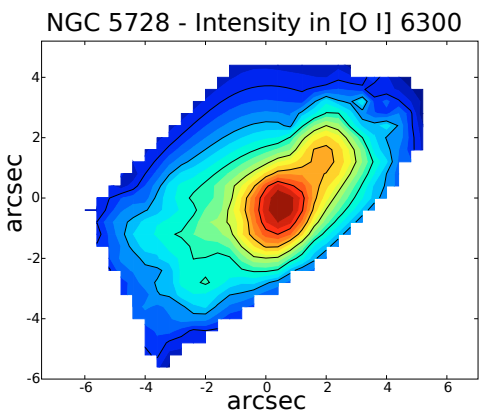

1.2

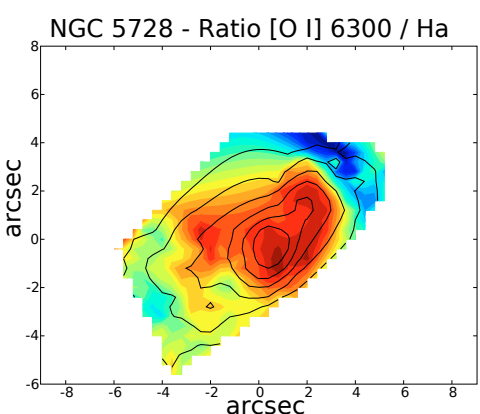

0.03

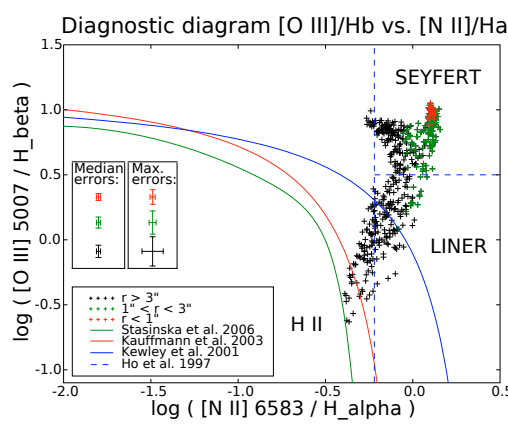

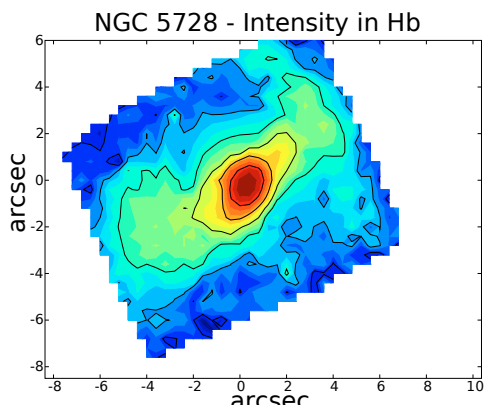

3

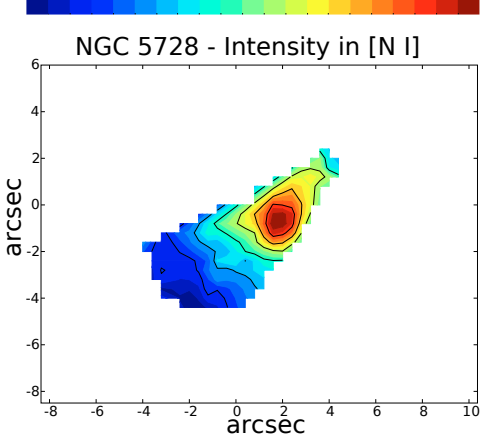

0.04

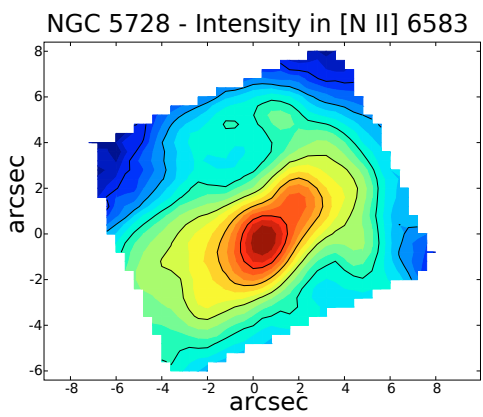

0.9

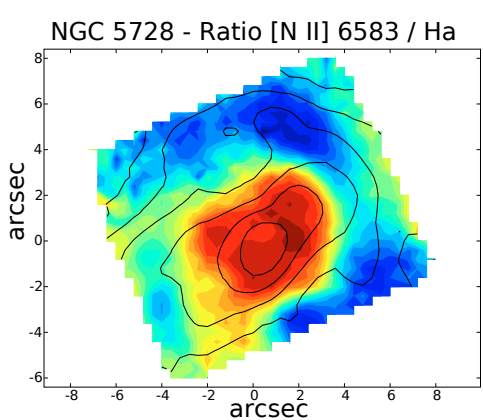

0.4

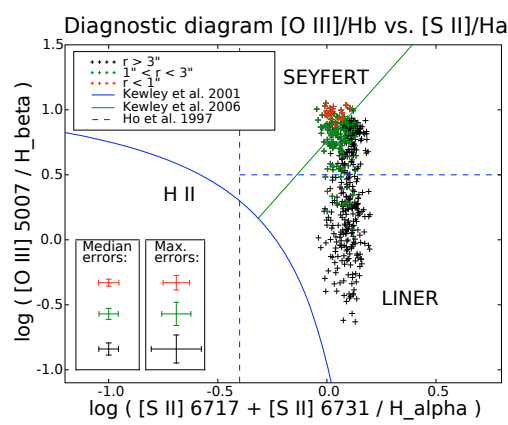

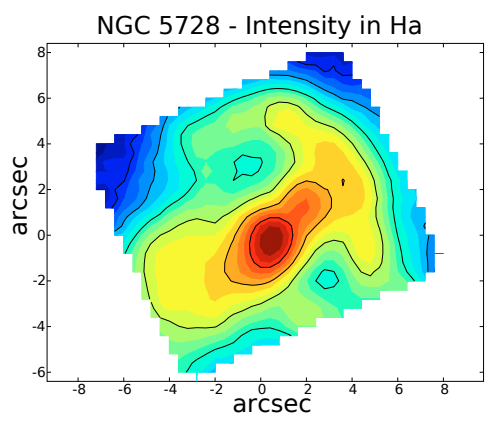
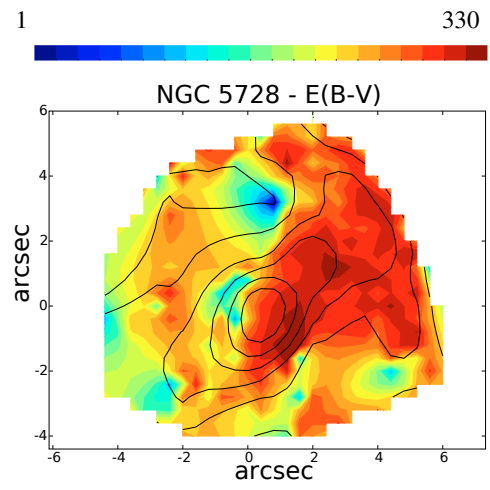

0

1.6

NGC 5728 - Intensity in [S II] 6717,6731

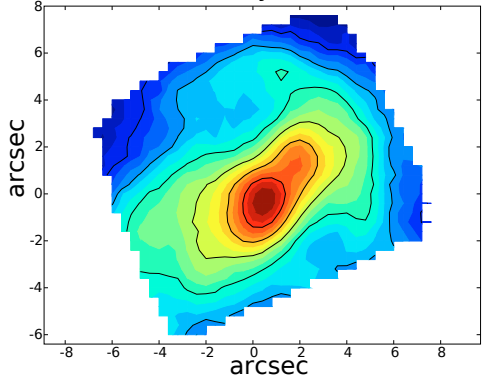

1.5

NGC 5728 - Ratio [S II] 6717,6731 / Ha

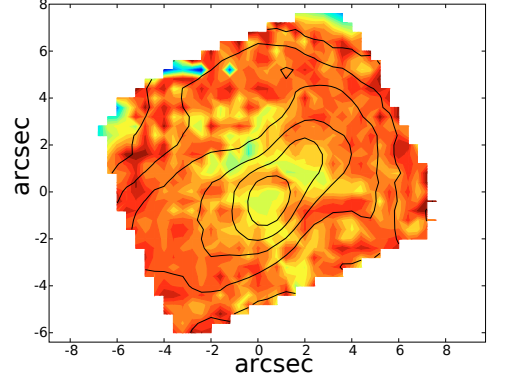

0.4

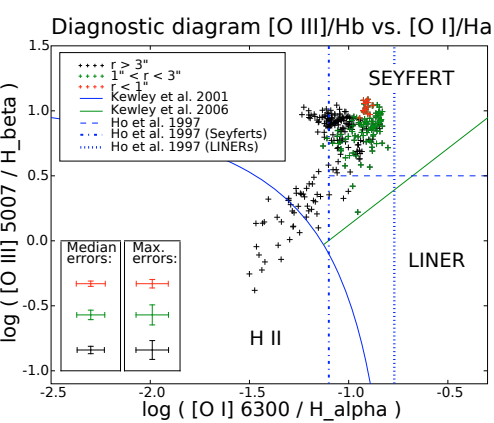

Fig. 22. NGC 5728. 


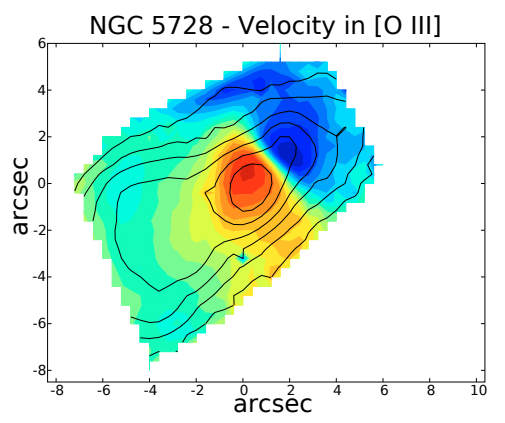

$-280$

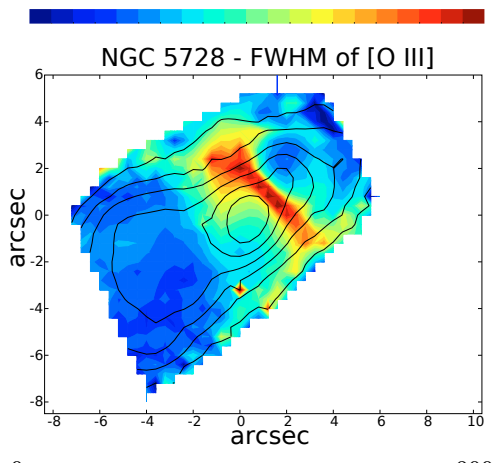

0

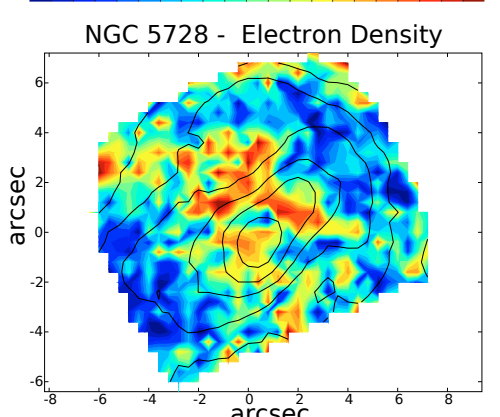

100

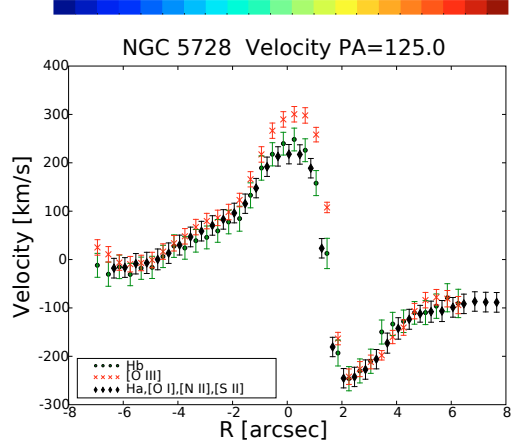

NGC 5728 - Diagnostic ratio [N II] 6583 / Ha

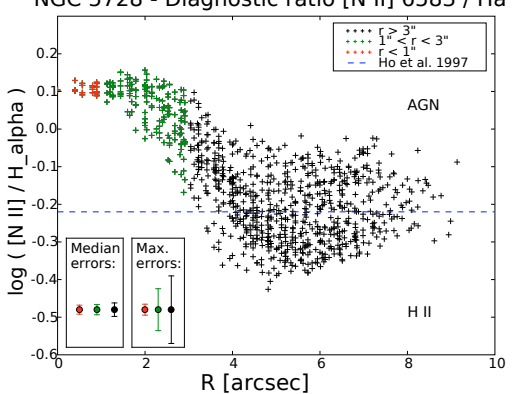

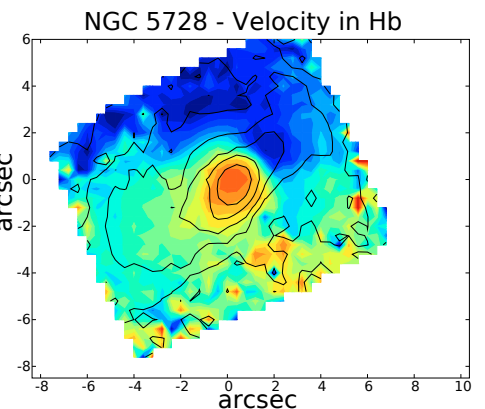

$-280$
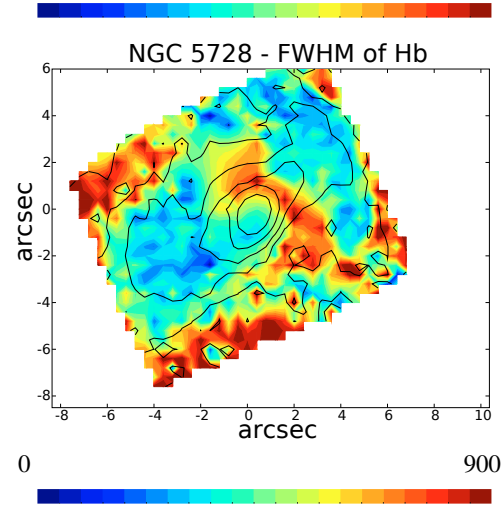

SSP mass Young/Total, $\mathrm{S} / \mathrm{N}=50$

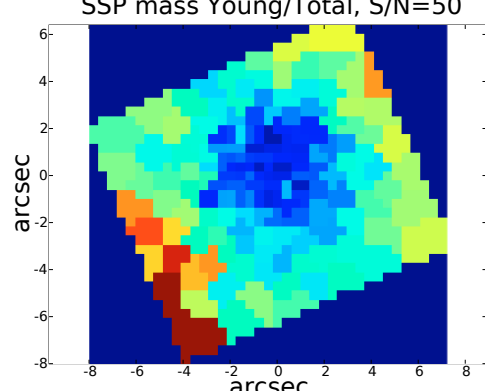

0

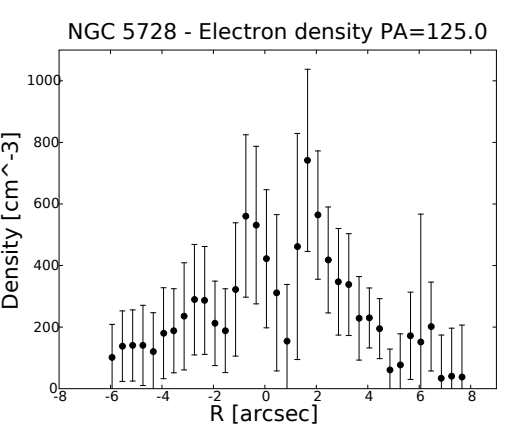

NGC 5728 - Diagnostic ratio [S II] 6717,6731/ Ha

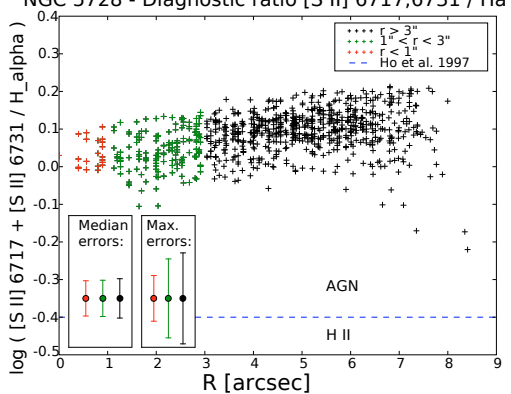

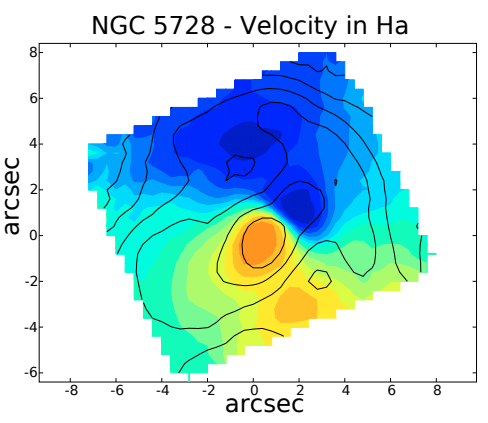

$-280$

320
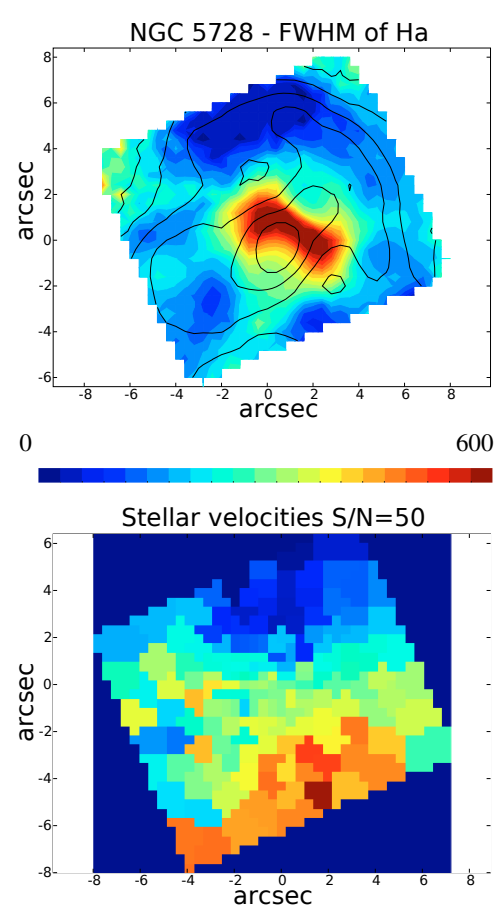

$-220$

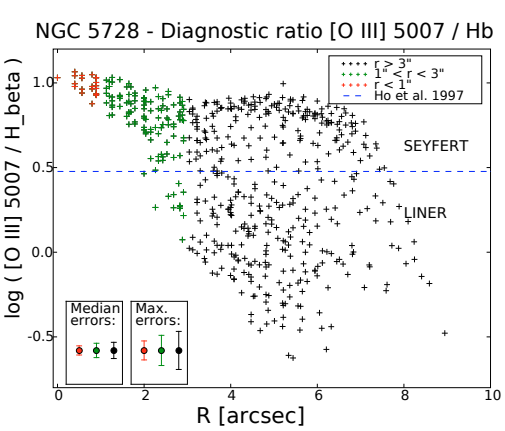

NGC 5728 - Diagnostic ratio [O I] $6300 / \mathrm{Ha}$

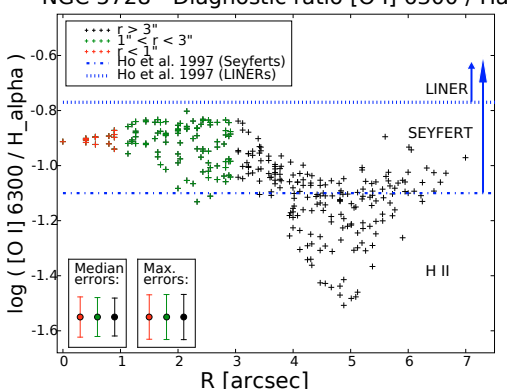

Fig. 23. NGC 5728. 

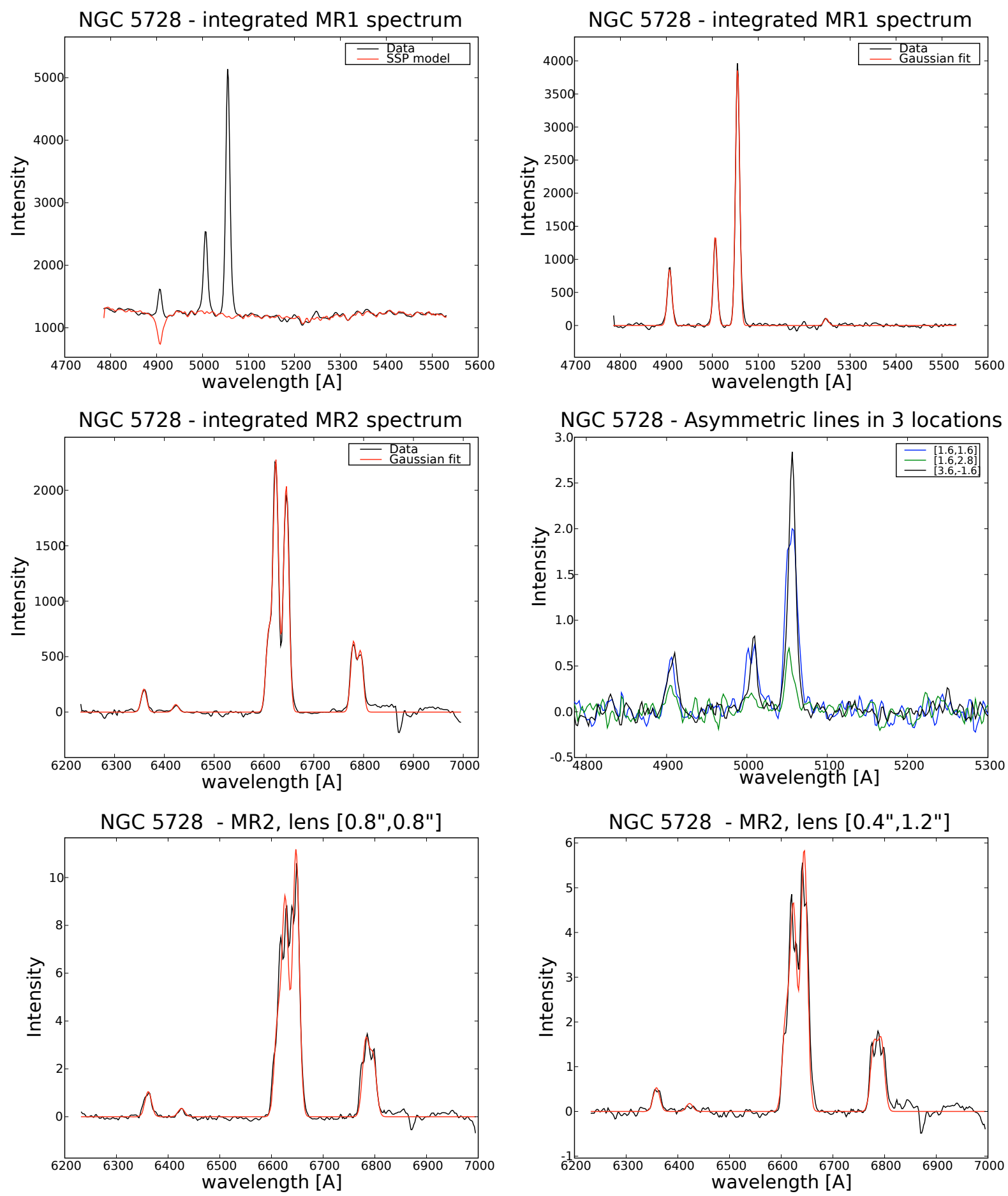

Fig. 24. NGC 5728.

perpendicular to the photometric major axis $\left(\mathrm{PA} \sim 45^{\circ}\right.$ in $[\mathrm{O}$ III] and PA $\sim 60^{\circ}$ in $\mathrm{H} \alpha$ ) and passing through the dynamic centre. Within our single-Gaussian model of emission profiles, the spatial region of asymmetric lines is represented by the largeFWHM area of the map (Fig. 23). We illustrate the multicomponent emission profiles with the varying importance of the individual components in Fig. 24.

Another interesting result is that for the dust distribution. The map of interstellar reddening plotted in Fig. 22 shows maximum extinction in the regions of emission maxima and the star-forming H II ring. The obscuration of the ionisation cones is also asymmetric and supports the interpretation of the north-west cone interacting with the galactic disc, and the southeast cone situated above the isc.

The stellar population ages resulting from our SSP modelling show a clear systematic distribution, with significant contributions (more than a few percent) from young stars only outside the central $\sim 600 \mathrm{pc}$.

\section{NGC 5929}

NGC 5929 forms an interacting pair with NGC 5930 (e.g., Bower et al. 1994). The galaxy hosts a linear radio source with 
two lobes separated by $1^{\prime \prime}$ along $\mathrm{PA}=60^{\circ}$ (Whittle et al. 1986; Ulvestad \& Wilson 1984b), close to the galaxy major axis at $\mathrm{PA}=45^{\circ}$ (Schmitt et al. 1997). Optical emission in [O III] and $\mathrm{H} \alpha+[\mathrm{N} \mathrm{II}]$ is in the form of cones co-aligned with the radio source, characterised by the opening angle of $80^{\circ}$ and an extent of 150-175 pc, and separated by a perpendicular dust lane (Bower et al. 1994; Schmitt \& Kinney 1996). Ferruit et al. (1997) performed IFU observations in $\mathrm{H} \alpha$, [N II], and [S II] with the TIGER spectrograph, and tested several models to account for the optical and radio emission. They place constraints on the interaction of the radio ejecta with the ambient gas, i.e., jet/cloud interaction, jet/ISM interaction, plasmon expansion, and the bow shock. None of the models were able to interpret the data satisfactorily.

Our observations were completed in one spectral domain (MR1) only. We found differences between the major axes of emission in $[\mathrm{O} \mathrm{III}]\left(\mathrm{PA} \sim 90^{\circ}\right)$ and in $\mathrm{H} \beta\left(\mathrm{PA} \sim 75^{\circ}\right)$, see Fig. 25. A similar discrepancy was seen in the zero-velocity curve orientation (approximately perpendicular to the emission major axes) in the maps of mean LOS velocities. However, the main features of the velocity fields, as well as their one-dimensional cuts, were consistent for both ions.

Our map of stellar velocities showed strong misalignment with the gas velocities: the stellar kinematic axis $\left(\mathrm{PA} \sim 30^{\circ}\right)$ was almost perpendicular to the symmetry axis of the gas. Moreover, the polarity of the stellar velocity field was reversed with respect to gas: in the north-east region, stars had receding motion, while the gas was approaching.

The LOS velocity dispersion maps show a stripe of maximum dispersion at $\mathrm{PA} \sim 160^{\circ}$ through the optical nucleus, approximately perpendicular to the photometric axis of $\mathrm{H} \beta$ (Fig. 25). The large-FWHM region is associated with asymmetric line profiles (Fig. 25), with a separation of $\sim 900 \mathrm{~km} \mathrm{~s}^{-1}$ between the line components.

The $[\mathrm{O} \mathrm{III}] / \mathrm{H} \beta$ intensity ratio distribution follows the morphology of [O III] emission, with maxima at both the nucleus and off the nucleus at $2^{\prime \prime}$ east, $2^{\prime \prime}$ west, and $4^{\prime \prime}$ west. The stellar populations show a clear pattern with a growing contribution of young populations toward the edges of the observed field.

\section{Discussion}

Despite the advances in 3D spectroscopic techniques, optical datacubes obtained with the resolution that we present in this paper remain rare. We have shown the wealth of information that the OASIS IFU data can provide, making it possible to address several important problems concerning AGN, while opening a perspective of promising innovative, detailed modelling. The relatively large number of emission lines contained in the data not only allow comparisons of NLR morphologies and kinematics for different ionic species, but also provide diagnostics for probing the physical conditions of the gas, based on the line intensity ratios. We have been able to estimate directly from the measured ratios both the electron density (the [S II] doublet ratio) and the interstellar reddening due to dust (the Balmer line ratio). The determination of other characteristics such as the ionisation parameter, metallicity, or temperature will require future modelling using spatially resolved diagnostic diagrams presented in this paper.

The emission data results have been compared with the stellar data, the kinematics in particular. We have also modelled the contributions of stellar populations of different ages and their variations across the observed field. This simultaneouslyacquired, rich information allows a more complex understanding of the structure and conditions in each of the observed Seyfert galaxies. In addition to studying each of the targets individually, we take advantage of having a uniform data set of Seyfert 2 galaxies and search for general trends in the physical properties. In this section, we summarise and discuss the obtained results in the context of the entire sample.

\subsection{Non-axisymmetric potentials}

One of the major goals has been to map the gas velocity fields in detail for different emission lines, and compare them with stellar velocities if possible, to understand more clearly the emission morphologies, the dominant dynamic processes at the galactic centre, and eventually the origin of the NLR gas. A number of scenarios have been proposed for NLRs that can be tested by spectroscopic studies. One of them concerns departures from the axial symmetry of the gravitational potential, which might be related to the nuclear activity and the gas transport across large distances toward the central galactic regions (e.g., Dumas et al. 2007; Schinnerer et al. 2000). On the other hand, signs of gas motion in non-coplanar orbits with the host galaxy may imply an external origin of the NLR gas, emphasising the role of mergers (e.g., Morse et al. 1998; Hunt \& Malkan 2004). Streaming motions toward the galactic centre might indicate a connection with AGN fuelling, and outflow motions are tested for their relation to the radio jets (e.g., Bicknell et al. 1998), or to the predicted thermal wind produced by the central torus (e.g., Krolik \& Begelman 1986; Balsara \& Krolik 1993).

Only one of the gas velocity fields obtained from our observations seems to be consistent with circular rotation in the central kiloparsec - NGC 449 (Mrk 1) - as far as our field-ofview allows us to judge. The mean LOS velocities of the ionised gas in seven of the eleven studied Seyfert 2 galaxies are characterised by S-shaped isovelocity contours in the central regions. This result supports the findings of Dumas et al. (2007), who reported velocity contour twists to be more common in the Seyfert galaxies than in quiescent ones. The S-shapes are in general signatures of non-axisymmetric galactic potentials containing bars, warps, or nuclear spirals (Roberts et al. 1979), and were successfully reproduced by these models in the case of several Seyfert galaxies e.g., by Veilleux et al. (1999a), Schinnerer et al. (2000), and Emsellem et al. (2006). Their interpretation of the velocity fields by motions in non-axisymmetric potentials thus provides an alternative to the radial flows often invoked to account for deviations from circular velocities. Degeneracies in the interpretation of non-circular motions were addressed analytically by Wong et al. (2004), who derived prescriptions of how to discriminate between bars, warps, and radial flows by means of Fourier analysis.

Beside the S-shaped velocity fields, another type of a nonaxisymmetric velocity pattern has been represented in our data in the case of NGC 262 (Mrk 348): two striking spots in the mean velocity map, suggesting a rotating disc/ring ( $\sim 300 \mathrm{pc}$ in radius) that is non-coplanar with the host-galaxy disc. Concerning outflows, we cannot provide direct evidence, even though at least two of the objects in our sample (NGC 4388, NGC 2992) have had an outflow component confirmed by several authors (e.g., Veilleux et al. 1999b; Márquez et al. 1998; Allen et al. 1999; Veilleux et al. 2001). We are partially limited by the small FOV, but a clearer understanding is expected from our future detailed kinematic modelling.

Except for one case (NGC 4388), the non-circular motions of gas do not have evident counterparts in the stellar velocity fields. This is unsurprising since gas, even in the absence of 

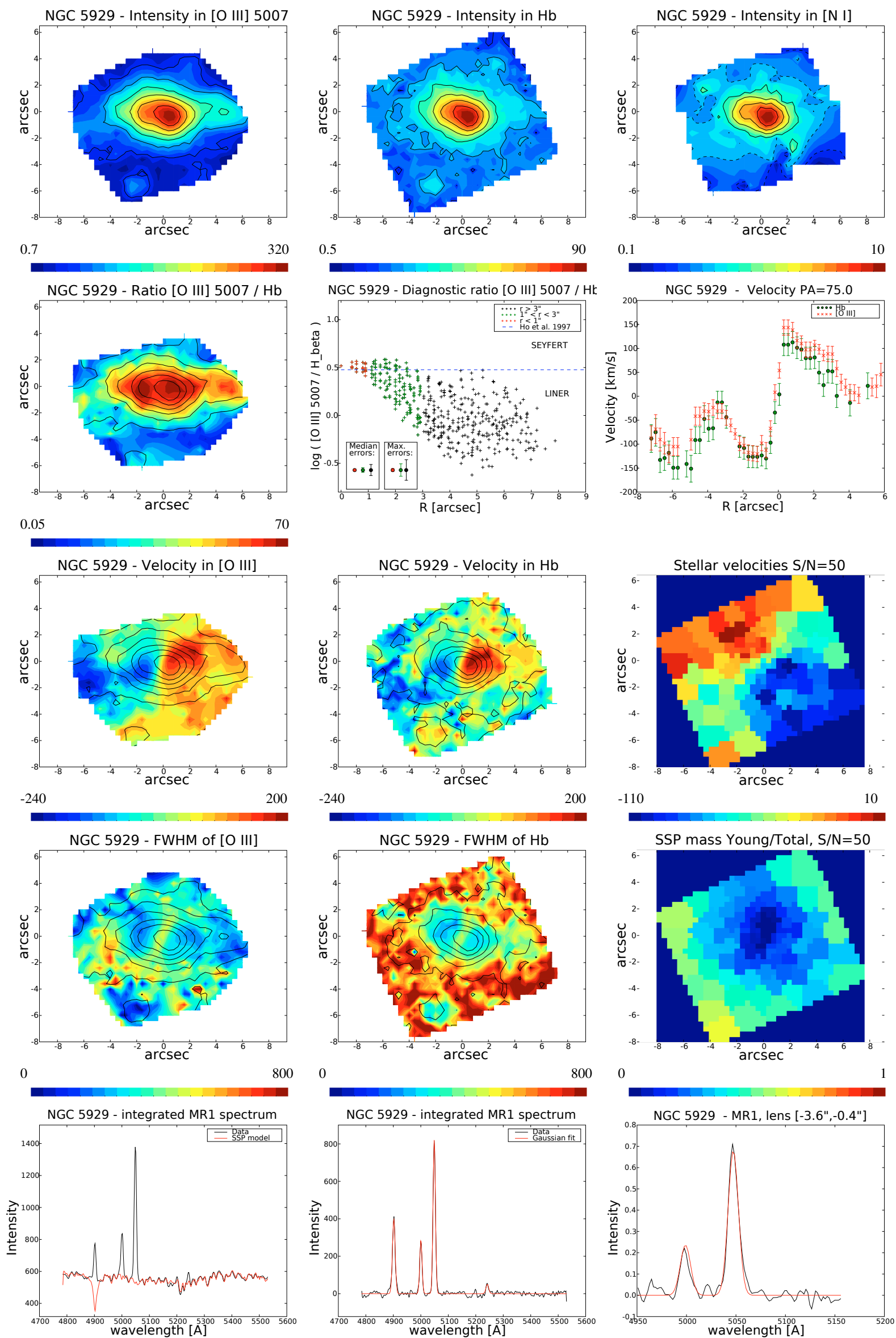

Fig. 25. NGC 5929. 
an AGN-driven outflow, is in general not expected to follow the same orbits as stars, due to its dissipative nature resulting in lower velocity dispersion, its higher responsiveness to nonaxisymmetric perturbations, possible ring formation, and a phase shift with respect to axes of stellar orbital families. A counterrotation or tilt with respect to the plane of the stellar disc are also plausible in the case of gas having an external origin (e.g., as a result of a minor merger). Observed misalignments between the kinematic axes of the stellar and gaseous components in active and non-active galaxies were discussed in detail by Dumas et al. (2007), who concluded that departures are both more frequent and significant in galaxies hosting an AGN. They also found that the closely aligned axes and a lack of isovelocity contour twists in gas were correlated with low-accretion rate AGN. Their results suggested a close relation between the AGN and the dynamics of the host.

\subsection{Nuclear rings}

Further type of evidence of a non-axisymmetric potential is the presence of nuclear rings. A small fraction of observed rings may be due to collisions or mergers of galaxies (e.g., Knapen et al. 2004), or to accretion of intergalactic gas, although the vast majority are probably resonance phenomena (Buta \& Combes 1996). Nuclear rings have been identified in approximately $20 \%$ of spiral galaxies (Knapen 2005). Our sample of Seyfert 2 galaxies possesses at least one object with a nuclear ring, reported previously (Wilson et al. 1993) and confirmed in our data: NGC 5728. We detect the ring in the surface brightness maps of the Balmer lines and low-ionisation lines of metals, while it is missing in [O III]. We have found it to be associated with high interstellar extinction, which suggests that it contains large amounts of dust.

Evidence of other rings might originate in the kinematic measurements, as was the case for NGC 5252 in Morse et al. (1998), and will be tested in future modelling. The strongest candidate to contain a ring is NGC 262 (Mrk 348) with the striking velocity pattern described in Sect. 5.1, not reported so far elsewhere. As suggested by Mulchaey et al. (1996a), NGC 262 possibly hosts another ring at $\sim 1 \mathrm{kpc}$. Marginal evidence of its detection was also found in our data, in the mean LOS velocity map of [O III], which displays systematic velocity pattern at the edges of our FOV that is qualitatively consistent with rotation in an opposite sense to that of the nuclear ring.

\subsection{NLR morphologies, cones}

The morphologies that we have obtained from analysis of different individual lines are not always identical or even aligned along the same axis. The misalignments between emission in [O III] and low-ionisation lines (e.g., NGC 449, NGC 5929) provide evidence of additional dynamic processes affecting the high-ionisation components of the gas, such as the radio jets that accelerate and/or ionise the gas.

Two of the galaxies (Mrk 1066, NGC 5728) show prominent regions detectable only in hydrogen and low-ionisation lines, which are virtually missing in [O III]: a nuclear ring in NGC 5728 (reported before e.g., by Wilson et al. 1993), and an off-centre region of diameter $\sim 500 \mathrm{pc}$ in Mrk 1066. The regions are characterised by a ratio $[\mathrm{O} \mathrm{III}] / \mathrm{H} \beta$ markedly lower than in the remainder of the observed field, and have therefore probably not been ionised by the AGN radiation. They are also associated with high interstellar reddening, which indicates a high amount of dust and possibly star formation, and hence an ionisation by young massive stars. Marginal support of this interpretation comes from the maps of stellar ages, which show relatively large contributions from young stars in the specified locations compared to the neighbouring regions. Ionisation by sources other than the active nucleus thus definitely play a role in the observed NLR morphology.

Directly related to the Unified Model of AGN (and the existence of the nuclear torus), the major puzzles about NLRs are their extent, the existence of ionisation cones, and the intrinsic geometry of the gas distribution. Significant evidence of cones was found in ground-based and HST images (e.g., Wilson et al. 1993; Falcke et al. 1998). The NLR structure parameters were analysed statistically for large samples by Mulchaey et al. (1996a) and Schmitt et al. (2003a), who both concluded that a surprisingly low number (with respect to the predictions of the Unified Model) of NLRs in Seyfert 2 nuclei have a major axis that is significantly longer than their minor axis. We reached the same conclusion, with the caveat that our results are influenced by both the small FOV and spatial resolution, mostly determined by the seeing conditions $\left(\sim 1^{\prime \prime}\right)$.

Mulchaey et al. (1996b) made predictions of NLR extents and morphologies for different observer viewing angles, and also for different intrinsic gas distributions ionised by the conical radiation field from the AGN. They considered two basic geometries of the gaseous region, a sphere and a disc, and tested their model predictions with data from a large imaging survey. In this context, our kinematic measurements clearly show that the observed NLRs are characterised by mostly rotational (although probably non-circular) motions, and are thus non-spherical.

\subsection{Alignment with radio emission}

Where detected, the elongated optical emission structures have been found to be aligned with radio emission data published in the literature, to within the measurement errors. This alignment infers an alignment between the torus and accretion-disc axes and/or an influence of the radio jet on the NLR gas dynamics and ionisation (Schmitt et al. 2003b). Our result confirms previous studies of the alignment between the two wavelength bands, demonstrated e.g., by Wilson \& Tsvetanov (1994), Nagar \& Wilson (1999), Falcke et al. (1998), Capetti et al. (1996), and Schmitt et al. (2003b).

As discussed above, NGC 449 and NGC 5929 of our Seyfert sample have different orientations of photometric axes in the high-ionisation and low-ionisation emission lines. The radiojet axis is consistent with [O III] emission in NGC 449, and with $\mathrm{H} \beta$ emission in NGC 5929. Conclusions are ambiguous for NGC 2273, which exhibits different shapes in its radio continuum emission at $20 \mathrm{~cm}$ and $6 \mathrm{~cm}$ wavelengths (Ulvestad \& Wilson 1984b). The $\mathrm{H} \beta$ emission appears to agree with the former and [O III] with the latter.

\subsection{Emission-line profiles}

We detect prominent splitting of emission lines in eight of the observed NLRs, usually limited to only part of the FOV, identified as a large- $F W H M$ region in the maps produced by our single-Gaussian line-profile modelling. In seven of the galaxies the region is of elongated shape approximately perpendicular to the NLR major axis and passes through the dynamic centre. Multi-component emission-line profiles have been reported in numerous NLR spectroscopic studies, but the integral-field 
spectroscopy permits us to map in detail the spatial variations in each component and to use the information in modelling the NLR structure and kinematics. The origin of the line splitting found in our data is unclear and will be addressed in future modelling of the observed objects. The most common interpretations include superposition of several kinematic components (e.g., Schommer et al. 1988), or expanding outflows (e.g., Capetti et al. 1999), rapidly rotating circumnuclear discs (e.g., Rubin et al. 1997), and also bar-induced non-circular motions (e.g., Veilleux et al. 2001; Bureau \& Athanassoula 1999; Kuijken \& Merrifield 1995).

\subsection{Electron densities}

We have estimated electron densities from the [S II] doublet in six galaxies of our sample for which data are available. In all cases, a non-uniform density distribution with a central peak has been found, the peak values ranging between $\sim 600 \mathrm{~cm}^{-3}$ and $\sim 1500 \mathrm{~cm}^{-3}$, and declining to $\sim 200 \mathrm{~cm}^{-3}$ outside the central $\sim 500 \mathrm{pc}$. The derived inhomogeneous gas distributions may have a strong effect on the estimates of global quantities, such as the NLR mass and the Strömgren radius.

The distributions are asymmetric and the aforementioned values are thus direction-dependent, although the overall results are consistent with long-slit observations (e.g., Bennert et al. 2006a; Fraquelli et al. 2003). More precise modelling is necessary to decide which of the values in the outer parts of the FOV (which are often relatively high) estimate reliably the intrinsic densities and which are an effect of measurement errors, to which the derived density values are highly sensitive. We also note that both the density values and the radial profiles are influenced by the method of derivation. The [S II] ratio is density-sensitive only within a limited range of values, and provides a density measure only in regions where these sulphur ions are present. The effects of observational errors are also non-negligible. We have also derived the densities based on the assumption of an electron temperature of $T=10000 \mathrm{~K}$, and different temperatures in different regions may therefore modify the absolute values and the spatial distribution of the computed densities (see e.g., Osterbrock 1989).

\subsection{Interstellar reddening}

The dust distribution may contribute significantly to the reconstruction of the galactic structure and the orientation of the observed nuclear emission regions with respect to the host galaxy, such as emission cones above or underneath the galactic disc. We have been able to estimate the dust distribution from the ratio of Balmer lines in 7 objects. The maximum extinction is usually connected with regions of maximum emission. Distinct offnuclear regions of high extinction have been found in NGC 262 (Mrk 348) and NGC 5728, consistent with low-ionisation regions such as the star-forming ring in NGC 5728. The dust distribution in NGC 5728 is an excellent example of asymmetry: the obscuration is much higher in the north-west emission cone than in the south-east part (see Fig. 22), which is consistent with the interpretation of Schommer et al. (1988), and Wilson et al. (1993), who predict the north-east cone to be interacting with the galactic disc.

The values of $E(B-V)$ that we have derived were based on the assumption that the intrinsic $\mathrm{H} \alpha / \mathrm{H} \beta$ ratio value equals 3.1, as is standard for AGN-excited gas due to hard radiation causing a relatively large transition zone (Osterbrock 1989). However, not all of the observed spectra necessarily originate in AGN-excited locations with an overabundance of high-energy photons compared with stellar spectra, and the intrinsic value of 2.86 would have been more appropriate. Therefore, reddening is underestimated in these locations.

The $E(B-V)$ that we have obtained is strongly dependent on the stellar population model applied to correct the Balmer emission lines for underlying absorption. The computed $E(B-V)$ values are significantly higher than those published e.g., by Bennert et al. (2006a), Raimann et al. (2003), Fraquelli et al. (2003), and Ho et al. (1997). The values that are common in the literature are of below one magnitude, in many cases determined by methods that differ from that of the Balmer decrement, and are thus independent of the stellar absorption models. We have tested the influence of the SSP models on the spatial distribution of dust, and on the $E(B-V)$ values. We have found that the spatial distributions remain unchanged qualitatively after applying the "minimum absorption correction" and using an old SSP as a stellar template. In contrast, the distribution differed when we did not correct for absorption and subtracted only the smooth continuum. We considered the no-absorption case to be non-realistic, and therefore conclude that our maps of $E(B-V)$ reflect the true character of the dust distribution. At the same time, the problem of the high reddening values was not solved by applying a different stellar model.

\subsection{Diagnostic line ratios}

The maps of diagnostic line ratios trace the spatial distribution of the gas physical characteristics and the properties of the ionisation source. The $[\mathrm{O} \mathrm{III}] / \mathrm{H} \beta$ ratio provides the most straightforward interpretation by tracing the gaseous media ionised by different sources projected along the line of sight. An outstanding example is NGC 4388 in Veilleux et al. (1999b), where $[\mathrm{O} \mathrm{III}] / \mathrm{H} \beta$ helps to separate between the gas in the galactic disc and the extraplanar material. An impact of the ionisation maps has been demonstrated in the case of NGC 5728, where the higher ionisation in one of the lobes has been used to advocate the inflow interpretation of the observed velocity field due to the position of the ionisation cones in relation to the galactic disc (Schommer et al. 1988).

In the objects that we have studied, maximum ionisation occurs in the vicinity of the galactic nucleus, and is consistent with a model of ionisation by AGN radiation. The isocontours traced by the $[\mathrm{O} \mathrm{III}] / \mathrm{H} \beta$ are not identical to those of the $[\mathrm{O}$ III] emission, and in approximately half of the sample the symmetry changes, which implies a contamination by other emission sources. The ionisation maps in general show more clearly defined axes than the [O III] emission, and have a more conical form than the [O III] maps, as far as our spatial resolution allows us to perceive. As already discussed in previous subsections, the ionisation maps have helped us to identify a ring in NGC 5728 and probably a star-forming region in Mrk 1066 . We have also confirmed high ionisation in the southern lobe of NGC 5728, reported by Schommer et al. (1988). The [O I]/H $\alpha$, [N II]/H $\alpha$, and [S II] $/ \mathrm{H} \alpha$ ratios have distributions that are off-centre in most of the observed cases.

We have constructed a set of three spatially resolved diagnostic diagrams for 7 of the 11 observed Seyfert 2s (for Mrk 34 only two have been possible). The distributions of points in the diagrams show considerable differences between the NLRs, reflecting important differences in their inner structures. We have included several theoretical curves (Kewley et al. 2001; Kauffmann et al. 2003; Stasińska et al. 2006; 
Table 4. Weights of stellar populations obtained by modelling of OASIS data, and compared to Cid Fernandes et al. (2004), on aperture $\sim 1^{\prime \prime}$. The symbols $\mu_{\mathrm{Y}}, \mu_{\mathrm{I}}, \mu_{\mathrm{O}}$ denote mass fractions of young, intermediate and old populations, respectively. Analogously for $\mu_{\text {young }}, \mu_{\text {old }}$ in our OASIS data.

\begin{tabular}{l|ll|lll}
\hline \hline Galaxy & \multicolumn{2}{|c|}{ OASIS data } & \multicolumn{2}{c}{ Cid Fernandes et al. (2004) } \\
& $\mu_{\text {young }}$ & $\mu_{\text {old }}$ & $\mu_{\mathrm{Y}}$ & $\mu_{\mathrm{I}}$ & $\mu_{\mathrm{O}}$ \\
\hline NGC 2992 & 0.09 & 0.91 & 0.0000 & 0.0260 & 0.9740 \\
NGC 3081 & 0.008 & 0.992 & 0.0000 & 0.0000 & 1.0 \\
NGC 4388 & 0.02 & 0.98 & 0.0002 & 0.0000 & 0.9998 \\
NGC 5728 & 0.06 & 0.94 & 0.0002 & 0.0699 & 0.9299 \\
\hline
\end{tabular}

Kewley et al. 2006) classifying emission-line objects based on their photoionisation source. However, the division inside the plots is only informative, since the models correspond to spectra integrated spatially over the nebula and are not adapted to the spatially resolved diagrams. A numerical ionisation modelling is necessary to achieve a correct interpretation. A strong Seyfert regime across the entire observed range of projected radial distances is detected in Mrk 34 and NGC 262 (Mrk 348). The opposite is true for Mrk 622, where all of the points lie below the Seyfert/LINER limit (which may be a consequence of the applied SSP models). The distributions of points are much less compact for Mrk 1066, NGC 449, NGC 2992, and NGC 5728, which have regions in the Seyfert regime as well as regions consistent with non-Seyfert ionisation. Numerical modelling similar to that carried out by Bennert et al. (2006a) will be necessary to determine the true edges of the NLRs.

\subsection{Stellar populations}

Stellar population modelling performed by using synthetic young and old SSPs has revealed varying contributions from young stars and their spatial distribution. The occurrence of young stars confirms results achieved in previous studies by several independent methods: wind lines from $\mathrm{O}$ and B stars in ultraviolet (UV) spectra (e.g., Heckman et al. 1997; González Delgado et al. 1998), and absorption lines of helium and of hydrogen in high-order Balmer series from $\mathrm{O}, \mathrm{B}$, and Wolf-Rayet stars (e.g., González Delgado et al. 1998, 2001). Statistical studies of Seyfert galaxies (e.g., Storchi-Bergmann et al. 2000; Joguet et al. 2001; Raimann et al. 2003) reported signatures of young populations in $30-50 \%$ of the observed Seyfert 2 galaxies, and significantly higher contributions $(\sim 20 \%$ in terms of flux in the nuclei) than in non-active galaxies. The detection of recent starburst in the nuclear regions of active galaxies may be closely related to the interplay between the host galaxy and the AGN, and to the triggering of the AGN. The highest contributions from young stars are present in AGN with high IR luminosity, which are interacting and have distorted inner morphologies (Cid Fernandes et al. 2001; Storchi-Bergmann et al. 2001).

We have compared the relative mass fractions of young stars obtained in our study with the results of Cid Fernandes et al. (2004). The comparison has been completed for spatially unresolved data, where we have simulated a $1^{\prime \prime}$ aperture to comply with Cid Fernandes et al. (2004) (see Table 4). The mass fractions that we obtain for the young population are of the same order as the combined fractions of young $\left(<10^{2} \mathrm{Myr}\right)$ and intermediate $\left(10^{2} \mathrm{Myr}-1.4 \mathrm{Gyr}\right)$ populations inferred by Cid Fernandes et al. (2004).

At least half of our Seyfert 2 sample exhibit clear spatial gradients in stellar population ages. In four galaxies (NGC 3081,
NGC 4388, NGC 5728, NGC 5929), old stars have been found to be dominant close to the dynamic centre or the location of peak emission, and a gradual increase in the contribution from young stars was measured outwards, which contradicts Raimann et al. (2003) who found an opposite trend. On the other hand, NGC 2992 contains its youngest populations close to the dust lane, and NGC 2273 has a maximal contribution from the young SSP north of the dynamic centre, which gradually decreases outwards. The remaining five galaxies (NGC 449, Mrk 34, NGC 262, Mrk 622, Mrk 1066) do not have high-quality stellar data, but the youngest populations have been fitted visibly in the central-most regions.

\section{Summary and conclusions}

We have analysed a set of 11 nearby Seyfert galaxies of type 2, observed with the integral-field spectrograph OASIS, which mapped the central $\sim 1 \mathrm{kpc}$ with medium spatial and spectral resolution. The central goal of our study was to obtain - from optical emission-line spectra - spatially resolved spectral diagnostics, and kinematical maps of the ionised gas. We have constructed, where possible:

- 2D maps of: surface brightness in individual lines, line ratios, velocity fields (mean LOS velocity and velocity dispersion), electron density, interstellar reddening, stellar mean LOS velocities, and relative mass fractions of young stellar populations.

- Spectral-diagnostic diagrams for line ratios sensitive to the source of ionisation (Seyfert, LINER and starburst regimes).

The 3D technique that we have applied provides a wealth of information that will be investigated in depth in forthcoming papers. The combined advantage of the relatively high spatial resolution and the length of the spectral range produce unprecedented details about the morphology, and both the kinematic and ionisation structure of the ionised gas of NLRs. We draw the following conclusions from the present study:

- Eighty percent of the observed galaxies exhibit S-shaped isovelocity contours in the mean LOS velocity maps of the ionised NLR gas. This suggests that non-axisymmetric potentials are probably common within the studied sample.

- We have detected a possible nuclear ring or a radial flow of gas in NGC 262 (Mrk 348), which was not reported before. It has been identified based on the mean LOS velocity maps derived from the observed emission lines.

- Electron densities usually reach their maximum values of $\sim 1000 \mathrm{~cm}^{-3}$ at the galactic nucleus, and decrease outwards.

- Dust is distributed unevenly in the central regions and will have to be included in a future detailed study of conditions in NLRs.

- Emission-line splitting has been identified in 8 out of 11 objects, confined to a clearly defined spatial region at the galactic centre, and in most cases elongated perpendicular to the major axis of emission.

- Our spatially resolved diagnostic diagrams show radial evolution from the Seyfert-like regime in the central regions to other regimes at larger distances.

- We find spatial gradients in stellar population ages, and confirm the presence of young stars. 


\section{Caption to Figs. 2-25}

Figures 2-25 contain (where available and relevant to the description of results and discussion, depending on individual galaxies):

a) 2D maps of surface brightness in emission lines of $\mathrm{H} \alpha$, $\mathrm{H} \beta$, [O III], [NI], [O I], [N II], and [S II] (in units of $10^{-19} \mathrm{~J} \mathrm{~s}^{-1} \operatorname{arcsec}^{-2} \mathrm{~m}^{-2}$ ), mean LOS velocity (in $\mathrm{km} \mathrm{s}^{-1}$ ), velocity dispersion $\left(F W H M\right.$, in $\left.\mathrm{km} \mathrm{s}^{-1}\right)$, line intensity ratios $[\mathrm{O} \mathrm{III}] / \mathrm{H} \beta,[\mathrm{N}$ II $] / \mathrm{H} \alpha,[\mathrm{S}$ II $] / \mathrm{H} \alpha,[\mathrm{O} \mathrm{I}] / \mathrm{H} \alpha$, reddening $E(B-V)$, and electron density.

b) Spatially resolved spectral-diagnostic diagrams.

c) Selected emission-line ratios as functions of projected galactocentric distance.

d) $1 \mathrm{D}$ cuts through mean LOS velocity and electron density along specific position angles.

e) Spectra - for selected individual spaxels, and/or integrated over circular apertures or the entire FOV - and their fits.

In the maps, north is up, east to the left. The black contours represent the surface brightness in the corresponding emission line for each of the derived quantities: in the case of mapped line ratios, the contours correspond to the forbidden line included in the ratio; for the electron density, they represent the sum of the two sulphur lines; and for the maps of interstellar reddening, they depict the surface brightness in $\mathrm{H} \alpha$. Any discrepancies between contours of the same species in different maps result from their re-computation for the new grid, where points of low $S / N$ in the other relevant line(s) were removed.

In the one-dimensional cuts, the projected radial-distance coordinates have been defined as increasing from east to west. Error bars in diagnostic diagrams and the emission-line ratio scatter plots have not been assigned to individual points, for clarity. Instead, median and maximum errors have been plotted for each of the defined spatial regions, in the corresponding colours. The spatially resolved spectral-diagnostic diagrams compare several classification schemes commonly applied to emission objects (originally with spatially non-resolved spectra). The two parallel lines for $[\mathrm{O} \mathrm{I}] / \mathrm{H} \alpha$ correspond to the values provided by $\mathrm{Ho}$ et al. (1997), $\log ([\mathrm{O} \mathrm{I}] / \mathrm{H} \alpha) \geq-1.1$ for Seyfert galaxies, and $\log ([\mathrm{O} \mathrm{I}] / \mathrm{H} \alpha) \geq-0.77$ for LINERs.

Acknowledgements. We would like to thank the anonymous referee for comments and suggestions. The work has been supported by the Centre for Theoretical Astrophysics (LC06014) of the Ministry of Education of the Czech Republic, by the Institutional Research Plan No. AV0Z10030501 of the Academy of Sciences of the Czech Republic, by the grant No. 205/03/H144/2003/02 of the Czech Science Foundation and by a Ph.D. fellowship provided by the French government for I.S. The research has made use of the NASA ADS database, HyperLeda database (http://leda. univ-lyon 1.fr), and NASA/IPAC Extragalactic Database (NED) which is operated by the Jet Propulsion Laboratory, California Institute of Technology, under contract with the National Aeronautics and Space Administration. We have made use of software packages for penalised pixel fitting and Voronoi tessellation developed by the SAURON consortium. Reduction software packages of ESO MIDAS (version 04SEPpl1.0) and IRAF have been used. IRAF is distributed by the National Optical Astronomy Observatories, which are operated by the Association of Universities for Research in Astronomy, Inc., under cooperative agreement with the National Science Foundation.

\section{References}

Allen, M. G., Dopita, M. A., Tsvetanov, Z. I., \& Sutherland, R. S. 1999, ApJ, 511,686

Antón, S., Thean, A. H. C., Pedlar, A., \& Browne, I. W. A. 2002, MNRAS, 336, 319

Antonucci, R. 1993, ARA\&A, 31, 473

Awaki, H., Koyama, K., Inoue, H., \& Halpern, J. P. 1991, PASJ, 43, 195
Bacon, R., Adam, G., Baranne, A., et al. 1995, A\&AS, 113, 347 Baldwin, J. A., Phillips, M. M., \& Terlevich, R. 1981, PASP, 93, 5 Balsara, D. S., \& Krolik, J. H. 1993, ApJ, 402, 109

Barbosa, F. K. B., Storchi-Bergmann, T., Cid Fernandes, R., Winge, C., \& Schmitt, H. 2006, MNRAS, 371, 170

Bennert, N., Jungwiert, B., Komossa, S., Haas, M., \& Chini, R. 2006a, A\&A, 456, 953

Bennert, N., Jungwiert, B., Komossa, S., Haas, M., \& Chini, R. 2006b, A\&A, 446, 919

Bicknell, G. V., Dopita, M. A., Tsvetanov, Z. I., \& Sutherland, R. S. 1998, ApJ, 495, 680

Boisson, C., \& Durret, F. 1986, A\&A, 168, 32

Boisson, C., Joly, M., Pelat, D., \& Ward, M. J. 2004, A\&A, 428, 373

Bower, G., Wilson, A., Morse, J. A., et al. 1995, ApJ, 454, 106

Bower, G. A., Wilson, A. S., Mulchaey, J. S., et al. 1994, AJ, 107, 1686

Braatz, J. A., Wilson, A. S., \& Henkel, C. 1994, ApJ, 437, L99

Bruzual, G., \& Charlot, S. 2003, MNRAS, 344, 1000

Bureau, M., \& Athanassoula, E. 1999, ApJ, 522, 686

Buta, R. 1990, ApJ, 351, 62

Buta, R., \& Combes, F. 1996, Fundamentals of Cosmic Physics, 17, 95

Buta, R., \& Purcell, G. B. 1998, AJ, 115, 484

Canalizo, G., Bennert, N., Jungwiert, B., et al. 2007, ApJ, 669, 801

Capetti, A., Axon, D. J., Macchetto, F., Sparks, W. B., \& Boksenberg, A. 1996, ApJ, 469, 554

Capetti, A., Axon, D. J., Macchetto, F. D., Marconi, A., \& Winge, C. 1999, ApJ, 516,187

Cappellari, M., \& Copin, Y. 2003, MNRAS, 342, 345

Cappellari, M., \& Emsellem, E. 2004, PASP, 116, 138

Cid Fernandes, R., Heckman, T., Schmitt, H., Delgado, R. M. G., \& Storchi-Bergmann, T. 2001, ApJ, 558, 81

Cid Fernandes, R., Gu, Q., Melnick, J., et al. 2004, MNRAS, 355, 273

Cid Fernandes, R., Mateus, A., Sodré, L., Stasińska, G., \& Gomes, J. M. 2005, MNRAS, 358, 363

Corbin, M. R., Baldwin, J. A., \& Wilson, A. S. 1988, ApJ, 334, 584

Dumas, G., Mundell, C. G., Emsellem, E., \& Nagar, N. M. 2007, MNRAS, 379, 1249

Emsellem, E., Greusard, D., Combes, F., et al. 2001, A\&A, 368, 52

Emsellem, E., Cappellari, M., Peletier, R. F., et al. 2004, MNRAS, 352, 721

Emsellem, E., Fathi, K., Wozniak, H., et al. 2006, MNRAS, 365, 367

Evans, I., Koratkar, A., Allen, M., Dopita, M., \& Tsvetanov, Z. 1999, ApJ, 521, 531

Falcke, H., Wilson, A. S., \& Simpson, C. 1998, ApJ, 502, 199

Falcke, H., Henkel, C., Peck, A. B., et al. 2000, A\&A, 358, L17

Ferruit, P., Pécontal, E., Wilson, A. S., \& Binette, L. 1997, A\&A, 328, 493

Ferruit, P., Wilson, A. S., \& Mulchaey, J. 2000, ApJS, 128, 139

Ferruit, P., Mundell, C. G., Nagar, N. M., et al. 2004, MNRAS, 352, 1180

Filippenko, A. V., \& Sargent, W. L. W. 1985, ApJS, 57, 503

Fraquelli, H. A., Storchi-Bergmann, T., \& Levenson, N. A. 2003, MNRAS, 341, 449

Girardi, L., Bressan, A., Bertelli, G., \& Chiosi, C. 2000, A\&AS, 141, 371

González Delgado, R. M., Heckman, T., Leitherer, C., et al. 1998, ApJ, 505, 174

González Delgado, R. M., Heckman, T., \& Leitherer, C. 2001, ApJ, 546, 845

Haniff, C. A., Wilson, A. S., \& Ward, M. J. 1988, ApJ, 334, 104

Hanson, C. G., Skinner, G. K., Eyles, C. J., \& Willmore, A. P. 1990, MNRAS, 242,262

Heckman, T. M., Sancisi, R., Balick, B., \& Sullivan, III, W. T. 1982, MNRAS, 199,425

Heckman, T. M., Gonzalez-Delgado, R., Leitherer, C., et al. 1997, ApJ, 482, 114

Henkel, C., Peck, A. B., Tarchi, A., et al. 2005, A\&A, 436, 75

Ho, L. C., \& Ulvestad, J. S. 2001, ApJS, 133, 77

Ho, L. C., Filippenko, A. V., \& Sargent, W. L. W. 1997, ApJS, 112, 315

Hunt, L. K., \& Malkan, M. A. 2004, ApJ, 616, 707

Iwasawa, K., Fabian, A. C., Ueno, S., et al. 1997, MNRAS, 285, 683

Joguet, B., Kunth, D., Melnick, J., Terlevich, R., \& Terlevich, E. 2001, A\&A, 380,19

Kauffmann, G., Heckman, T. M., Tremonti, C., et al. 2003, MNRAS, 346, 1055 Kay, L. E. 1994, ApJ, 430, 196

Kewley, L. J., Dopita, M. A., Sutherland, R. S., Heisler, C. A., \& Trevena, J. 2001, ApJ, 556, 121

Kewley, L. J., Groves, B., Kauffmann, G., \& Heckman, T. 2006, MNRAS, 372, 961

Knapen, J. H. 2005, A\&A, 429, 141

Knapen, J. H., Whyte, L. F., de Blok, W. J. G., \& van der Hulst, J. M. 2004, A\&A, 423, 481

Krolik, J. H., \& Begelman, M. C. 1986, ApJ, 308, L55

Kuijken, K., \& Merrifield, M. R. 1995, ApJ, 443, L13

Kukula, M. J., Ghosh, T., Pedlar, A., \& Schilizzi, R. T. 1999, ApJ, 518, 117

Laurikainen, E., Salo, H., \& Buta, R. 2004, ApJ, 607, 103 
Lebrun, F., Ballet, J., Paul, J., et al. 1992, A\&A, 264, 22

Márquez, I., Boisson, C., Durret, F., \& Petitjean, P. 1998, A\&A, 333, 459

Martini, P., Regan, M. W., Mulchaey, J. S., \& Pogge, R. W. 2003, ApJ, 589, 774

Mazzarella, J. M., \& Boroson, T. A. 1993, ApJS, 85, 27

McDermid, R. M., Emsellem, E., Shapiro, K. L., et al. 2006, MNRAS, 373, 906

Miller, J. S., \& Goodrich, R. W. 1990, ApJ, 355, 456

Moiseev, A. V., Valdés, J. R., \& Chavushyan, V. H. 2004, A\&A, 421, 433

Morse, J. A., Raymond, J. C., \& Wilson, A. S. 1996, PASP, 108, 426

Morse, J. A., Cecil, G., Wilson, A. S., \& Tsvetanov, Z. I. 1998, ApJ, 505, 159

Mulchaey, J. S., Wilson, A. S., \& Tsvetanov, Z. 1996a, ApJS, 102, 309

Mulchaey, J. S., Wilson, A. S., \& Tsvetanov, Z. 1996b, ApJ, 467, 197

Nagar, N. M., \& Wilson, A. S. 1999, ApJ, 516, 97

Nagar, N. M., Wilson, A. S., Mulchaey, J. S., \& Gallimore, J. F. 1999, ApJS, 120,209

Neff, S. G., \& de Bruyn, A. G. 1983, A\&A, 128, 318

Osterbrock, D. E. 1989, Astrophysics of gaseous nebulae and active galactic nuclei, University of Minnesota (Mill Valley, CA: University Science Books)

Osterbrock, D. E., \& Dahari, O. 1983, ApJ, 273, 478

Peck, A. B., Henkel, C., Ulvestad, J. S., et al. 2003, ApJ, 590, 149

Pécontal-Rousset, A., Bacon, R., Copin, Y., et al. 2004a, in Astronomical Data Analysis Software and Systems (ADASS) XIII, ed. F. Ochsenbein, M. G. Allen, \& D. Egret, ASP Conf. Ser., 314, 491

Pécontal-Rousset, A., Copin, Y., \& Ferruit, P. 2004b, AN, 325, 163

Pérez, I., Fux, R., \& Freeman, K. 2004, A\&A, 424, 799

Phillips, M. M., \& Malin, D. F. 1982, MNRAS, 199, 905

Pogge, R. W. 1988, ApJ, 332, 702

Pogge, R. W. 1989, ApJ, 345, 730

Raimann, D., Storchi-Bergmann, T., González Delgado, R. M., et al. 2003, MNRAS, 339, 772

Riffel, R. A., Storchi-Bergmann, T., Winge, C., et al. 2008, MNRAS, 385, 1129

Roberts, Jr., W. W., Huntley, J. M., \& van Albada, G. D. 1979, ApJ, 233, 67

Rousset, A. 1992, Ph.D. Thesis, Univ. J. Monnet de Saint-Etienne

Rubin, V. C., Kenney, J. D. P., \& Young, J. S. 1997, AJ, 113, 1250

Ruiz, J. R., Crenshaw, D. M., Kraemer, S. B., et al. 2005, AJ, 129, 73

Sánchez, S. F., Cardiel, N., Verheijen, M. A. W., et al. 2007, A\&A, 465, 207

Sarzi, M., Falcón-Barroso, J., Davies, R. L., et al. 2006, MNRAS, 366, 1151

Schinnerer, E., Eckart, A., Tacconi, L. J., Genzel, R., \& Downes, D. 2000, ApJ, 533,850

Schmitt, H. R., \& Kinney, A. L. 1996, ApJ, 463, 498

Schmitt, H. R., Kinney, A. L., Storchi-Bergmann, T., \& Antonucci, R. 1997, ApJ, 477, 623

Schmitt, H. R., Storchi-Bergmann, T., \& Fernandes, R. C. 1999, MNRAS, 303, 173
Schmitt, H. R., Donley, J. L., Antonucci, R. R. J., Hutchings, J. B., \& Kinney, A. L. 2003a, ApJS, 148, 327

Schmitt, H. R., Donley, J. L., Antonucci, R. R. J., et al. 2003b, ApJ, 597, 768

Schommer, R. A., Caldwell, N., Wilson, A. S., et al. 1988, ApJ, 324, 154

Shuder, J. M., \& Osterbrock, D. E. 1981, ApJ, 250, 55

Simpson, C., Mulchaey, J. S., Wilson, A. S., Ward, M. J., \& Alonso-Herrero, A. 1996, ApJ, 457, L19

Sosa-Brito, R. M., Tacconi-Garman, L. E., Lehnert, M. D., \& Gallimore, J. F. 2001, ApJS, 136, 61

Stasińska, G. 2009, in The Emission Line Universe, ed. J. Cepa, 1

Stasińska, G., Cid Fernandes, R., Mateus, A., Sodré, L., \& Asari, N. V. 2006, MNRAS, 371, 972

Stone, Jr., J. L., Wilson, A. S., \& Ward, M. J. 1988, ApJ, 330, 105

Storchi-Bergmann, T., Bica, E., \& Pastoriza, M. G. 1990, MNRAS, 245, 749

Storchi-Bergmann, T., Raimann, D., Bica, E. L. D., \& Fraquelli, H. A. 2000, ApJ, 544, 747

Storchi-Bergmann, T., González Delgado, R. M., Schmitt, H. R., Cid Fernandes, R., \& Heckman, T. 2001, ApJ, 559, 147

Takano, S., \& Koyama, K. 1991, PASJ, 43,

Tremonti, C. A., Heckman, T. M., Kauffmann, G., et al. 2004, ApJ, 613, 898

Ulvestad, J. S., \& Wilson, A. S. 1984a, ApJ, 278, 544

Ulvestad, J. S., \& Wilson, A. S. 1984b, ApJ, 285, 439

Ulvestad, J. S., \& Wilson, A. S. 1989, ApJ, 343, 659

Urry, C. M., \& Padovani, P. 1995, PASP, 107, 803

van Driel, W., \& Buta, R. J. 1991, A\&A, 245, 7

Veilleux, S., \& Osterbrock, D. E. 1987, ApJS, 63, 295

Veilleux, S., Goodrich, R. W., \& Hill, G. J. 1997, ApJ, 477, 631

Veilleux, S., Bland-Hawthorn, J., \& Cecil, G. 1999a, AJ, 118, 2108

Veilleux, S., Bland-Hawthorn, J., Cecil, G., Tully, R. B., \& Miller, S. T. 1999b, ApJ, 520, 111

Veilleux, S., Shopbell, P. L., \& Miller, S. T. 2001, AJ, 121, 198

Ward, M., Penston, M. V., Blades, J. C., \& Turtle, A. J. 1980, MNRAS

Wehrle, A. E., \& Morris, M. 1988, AJ, 95, 1689

Whittle, M., Haniff, C. A., Ward, M. J., et al. 1986, MNRAS, 222, 189

Wilson, A. S., \& Tsvetanov, Z. I. 1994, AJ, 107, 1227

Wilson, A. S., Braatz, J. A., Heckman, T. M., Krolik, J. H., \& Miley, G. K. 1993 , ApJ, 419, L61

Wong, T., Blitz, L., \& Bosma, A. 2004, ApJ, 605, 183

Wozniak, H., Friedli, D., Martinet, L., Martin, P., \& Bratschi, P. 1995, A\&AS,

111,115

Xanthopoulos, E., \& Richards, A. M. S. 2001, MNRAS, 326, L37

Yasuda, N., Fukugita, M., \& Okamura, S. 1997, ApJS, 108, 417

Zhang, E.-P., \& Wang, J.-M. 2006, ApJ, 653, 137 